\title{
DIRECT SIMULATION OF MULTIPHASE FLOWS WITH \\ MODELING OF DYNAMIC INTERFACE CONTACT ANGLE
}

\author{
Seungwon Shin1, Jalel Chergui2 and Damir Juric2 \\ 1 Department of Mechanical and System Design Engineering \\ Hongik University \\ Seoul, 121-791 Korea \\ 2 Laboratoire d'Informatique pour la Mécanique et les Sciences de l'Ingénieur (LIMSI), \\ Centre National de la Recherche Scientifique (CNRS), \\ CNRS-UPR 3251, Université Paris Saclay, Bât. 508, Rue John von Neumann \\ Campus Universitaire d'Orsay \\ 91405 Orsay, France
}

*Corresponding Author:

Seungwon Shin, $\mathrm{PhD}$

Associate Professor

Department of Mechanical and System Design Engineering

Hongik University

Sangsu-dong, 72-1, Mapo-gu

Seoul, 121-791, Korea

Phone: 82-2-320-3038

FAX: 82-2-322-7003

E-Mail: sshin@hongik.ac.kr 


\begin{abstract}
We describe a modeling technique for dynamic contact angle between a phase interface and a solid wall using a Generalized Navier Boundary Condition (GNBC) in the context of a Front-Tracking based multiphase method. The contact line motion is determined by the Generalized Navier-Slip boundary condition in order to eliminate the infinite shear stress at the contact line. Applying this slip boundary condition only to the interface movement with various slip ratios shows good agreement with experimental results compared to allowing full fluid slip along the solid surface. The interface slip model performs well on grid convergence tests using both the slip ratio and slip length models. A detailed energy analysis was performed to identify changes in kinetic, surface and potential energies as well as viscous and contact line dissipation with time. A friction coefficient for contact line dissipation was obtained based on the other computed energy terms. Each energy term as well as the friction coefficient were compared for different grid resolutions. The effect of varying the slip ratio as well as the contact angle distribution vs contact line speed was analyzed. The behavior of drop impact on a solid wall with different advancing and receding angles was investigated. Finally, the proposed dynamic contact model was extended to three-dimensions for large-scale parallel calculations. The impact of a droplet on a solid cylinder was simulated to demonstrate the capabilities of the proposing formulation on general solid structures. Widely different contact angles were tested and showed distinctive characteristic behavior clearly.
\end{abstract}

Keywords: multiphase flow, numerical simulation, front-tracking method, contact line modeling, dynamic contact angle 


\section{INTRODUCTION}

Contact line behavior in multiphase flows is very important in many natural as well as engineering processes which include capillary dynamics, wetting or droplet impact on solid surfaces. Recently the droplet jumping phenomenon during condensation has drawn attention since it can considerably promote heat transfer from a wall without additional energy input [1]. This self-induced jumping of micro-droplets is strongly controlled by surface tension effects as well as contact line dynamics which are both very important to the overall kinematics. Due to experimental constraints, the study of contact line dynamics has usually been restricted to measurements of general characteristics of contact line motion such as wetting area or speed. Numerical simulations can thus be useful for in-depth investigations of the underlying physics associated with contact line dynamics.

Recent advances in three-dimensional numerical modeling of multiphase flows have used various modeling techniques such as those in the category of front-capturing type methods: Volume of Fluid (VOF) [2], Level Set [3], and Phase Field [4] methods. These methods capture interface motion through the advection of an auxiliary Eulerian scalar field i.e. local volume fraction (VOF), distance function (Level Set) or Phase Field. Another category of numerical techniques for handling phase interfaces is based on Front-Tracking [5] and uses a dedicated Lagrangian interface structure due to its accuracy in modeling interface dynamics and surface forces. However due to algorithmic difficulty in maintaining interface connectivity of the triangular Lagrangian mesh, the classic Front-Tracking method is perceived to be difficult to generalize to three-dimensional simulations. Recently, hybrid capturingtracking methods have been developed which retain the advantages associated with each technique. Here we use one such hybrid, the Level Contour Reconstruction method [6] which combines characteristics of Front Tracking and Level Set methods. A more detailed description of the LCRM can be found in the next section.

Despite recent improvements in the accuracy and efficiency of two-phase flow simulations, most investigations have been focused on cases with isolated drops or bubbles. However, when the phase interface touches a solid wall, a contact line will be formed which will further complicate multiphase 
modeling. An accurate description of the contact line motion is essential to the correct modeling of such two-phase flows. Using a general no-slip condition, the contact line is pinned to a specific location at the wall and will thus unnaturally generate infinite shear stress at the wall. To prevent this unbounded shear stress condition, there are generally two different approaches for handling contact line dynamics. The first is to allow interface slip near the contact line, i.e. a hydrodynamic model originally proposed by Hocking [7]. Cox [8] divided the contact zone into micro and macro regions and considered an intermediate region between them. Fluid slip is allowed in the micro region and depends on a relation between the dynamic contact angle and slip velocity from an asymptotic solution. The second approach uses adsorption and desorption relations from molecular theory near the contact line [9], i.e. molecular kinetic modeling. Both approaches can match experimental results successfully with some adjustments and have been applied to resolve contact line problems in various ways.

Huang et al. [10] investigated bubble movement in shear flow near the wall using a Front-Tracking method. A conventional Navier-slip condition, where contact line slip is proportional to the velocity gradient, was used. The contact line speed was computed using a friction parameter which is proportional to the difference between the contact angle and the advancing or receding angles. The dynamic contact angle was determined by a linear model. Local forces were introduced based on the moving contact angle and contact line speed. Ren et al. [11] considered non-Newtonian behavior near the contact line where a linear response is no longer valid. They pointed out that an unbalanced Young's stress is necessary for a more accurate computation. The conventional no-slip condition is still valid away from the contact line but a slip boundary condition is required near the contact line. They modified the contact model which accounts for tangential as well as normal force balances near the contact line. They proposed an effective boundary condition from molecular dynamics (MD) simulations and applied it to their continuum model. Afkhami et al. [12] imposed a slip boundary condition implicitly using a VOF method. The Navier-slip condition was used at the wall and this slip condition was applied to the entire wall surface. A grid converged solution was obtained using a slip length related to the grid size. To obtain a fully converged solution, a sufficient slip length was necessary. The fluid motion in the viscous region near the contact line was replaced by an analytic asymptotic solution considering the grid independent behavior of fluid flow with small Capillary number $(<0.1)$. The difference between 
macro and micro angle was used to generate a functional relation between them. From the relation of Cox [8] with an appropriate choice of length scale, the apparent contact angle from the simulation can be cast into a micro-scale angle. Thus, contact line speed from the micro angle becomes resolution independent even if the apparent contact angle would change depending on the grid resolution. This model was applied to simulate the case of a plate withdrawn from a liquid pool.

Dupont and Legendre [13] devised a contact model by enforcing an appropriate color function value at ghost points inside the wall which satisfy contact angle constraints. Contact angle hysteresis was considered and the macro contact angle was related to the micro-scale value using Cox's relation [8]. To expedite the simulation, a fitted function of Cox's relation was used. The contact line speed was not given but computed from the momentum equation during the simulation. The contact angle which satisfies the local momentum balance was iteratively searched for and the advancing or receding angle was chosen depending on its direction of movement. Various benchmark tests were performed including droplet dynamics on an inclined wall.

Sui and Spelt [14] proposed a contact model based on the Level Set method. The model relates the micro and macro contact angles using Cox's relation [8]. They argued that the macro contact angle can be applied to the contact model directly if sufficient grid resolution is available so that the viscous inner region can be captured. With limited grid resolution, there will be a difference between macro and micro contact angles and thus a proper relation needs to be provided. The Navier slip condition was applied to the entire wall and contact angle hysteresis was not considered. A simple interfacial source term was used without modeling of the force balance in the normal direction. The unbalanced Young's stress was not calculated directly but rather implicitly using a macro to micro contact angle relation. Detailed grid convergence tests were performed and showed fully converged behavior for the macro to micro model.

Yamamoto et al. [15] modified the Generalized Navier Boundary Condition originally proposed by Qian et al. [16] for the Front-Tracking method. The interfacial Young's stress at the contact line was considered to play a very important role based on evidence from MD simulations [17]. They applied their model to the problem of meniscus rise. Numerical results were compared with experimental measurement and matched very well. However, additional computations near the contact line were 
necessary in order to apply the GNBC. They also upgraded their contact line formulation by including a relation between micro and macro contact angles based on Cox's relation [19] and recently extended the method to three-dimensions to simulate contact line behavior upon passing a defect on vertical wall [39]. Zhang et al. [18] applied the GNBC to the Phase Field method and investigated the drop impact process in detail. A wide range of impact regimes including adherence, bouncing, and splashing were considered with various input conditions.

Due to the modeling complexity, typically the steady state or transient toward steady state behavior of the contact line dynamics was analyzed as discussed above. On the other hand, fully dynamic processes in contact line motion were also intensively studied [19-22]. In these cases, simpler models have been used since movement of the contact line is relatively rapid and contact angle hysteresis must be accounted for. Yokoi et al. [23] numerically analyzed the drop impact problem using a CLSVOF method and compared the simulation results with experiment. Using an extension velocity, contact angle hysteresis was applied without explicitly locating the contact point. The slip length was not considered since the no-slip condition does not generate a singularity in their formulation. A contact angle relation as a function of contact line speed was devised using Tanner's law [24] for flow in the capillary regime. In the inertia dominant regime, the contact angle was fixed to either the advancing or receding angle. They tested various input conditions for contact angle profiles depending on contact line speed. They found that the simulation results match experiments very well with similar functional relations between contact line speed and contact angle.

Most studies of contact line behavior in dynamic situations use a conventional Navier-slip condition with treatment of contact angle depending on the advancing and receding dynamics. The front capturing approach is widely used for dynamic contact modeling due to its simplicity. A scalar field representing interface motion can be specified to certain values which satisfy the given contact angle associated with each method. In Front-Tracking type methods, a Heaviside function generated from the Lagrangian interface can be specified similarly. In this study, we introduce an extended interface at the contact line to control contact angle hysteresis more accurately by fully utilizing FrontTracking's precise interface positioning [25]. We show that the extended interface naturally accounts for the GNBC in the context of the Front Tracking method. Our ultimate goal is to generalize the contact 
model for fully three-dimensional flows with complex solid structures in a simple yet robust formulation. Since identifying the location of the contact line (3D) or contact point (2D) is very straightforward in Front Tracking, the extended interface can easily be defined even in the presence of complex solid structures.

The current paper is organized as follows; we first discuss the general description of our interface tracking method (the Level Contour Reconstruction method) in the next section. Then we provide a detailed description of the contact line model including the extended surface concept and wall slip. The overall numerical scheme is briefly described. We then present verification of our contact line model for the case of drop impact on a flat wall in axisymmetric geometry. Various slip conditions are investigated and grid convergence tests are provided. For a test of the full three-dimensional contact model on complex solid structures, we demonstrate the simulation of a droplet impact on a stationary cylinder for three different contact angle conditions. 


\section{NUMERICAL FORMULATION}

\subsection{Interface tracking method}

To track an evolving and deforming interface, Front Tracking type methods use a separate Lagrangian interface mesh composed of line segments (2D) or triangular elements (3D). This mesh is contained within a fixed Eulerian mesh upon which the fluid variables are discretized. The Lagrangian interface mesh makes the Front Tracking method very effective in precisely describing the phase boundary and setting capillary forces. However, the inherent difficulty in classic Front Tracking, especially in three dimensions, is maintaining uniformity of the interface elements and allowing for topology change. On the other hand, front capturing type methods implicitly locate the phase boundary by solving an additional Eulerian advection equation for the scalar field of a phase indicator such as the local volume (Volume of Fluid) or distance function (Level Set). Since this additional scalar field is defined on the same grid as the velocity and pressure, the formulation is relatively easy and straightforward. Some drawbacks such as ensuring continuity (VOF) and diffusivity (LS) of the interface were noticed and various remedies have been suggested to overcome those difficulties.

In this article, we use the Level Contour Reconstruction Method (LCRM) [6] to track the moving interface. The LCRM hybridizes the Front Tracking and Level Set methods to combine the advantages of each method. The basic concept in the LCRM is depicted in Fig. 1. The LCRM basically tracks an additional Lagrangian interface similar to classic Front Tracking but it also computes a distance function directly from the interface location (the detailed procedure can be found in [26]). Interface elements are periodically refreshed by repositioning them along the contour of a specific value of the distance function thus endowing the technique with Level Set characteristics (see Fig. 1). Interface reconstruction from the distance function involves drawing iso-contour lines in each reconstruction cell. To avoid ambiguity in drawing the iso-contour lines, the rectangular reconstruction cell has been divided into two triangles. Along the reconstruction cell edges, we locate the zero distance point and connect the interface line when two points are located at the cell edges (see Fig. 1). The regenerated interface elements maintain a certain distance from each other preventing excessive coagulation or dispersion. 
The underlying grid cells used for the primary variables of velocity, pressure, and temperature are divided into tetrahedral cells for tetra-marching reconstruction. The advantage of tetrahedral cells is that the contour reconstruction in each cell is guaranteed to be unique. A more precise allocation of the interface elements can be obtained using a high order reconstruction technique [27]. The curvature computation was improved [28] to eliminate spurious currents even in cases where capillary forces are dominant as in microscale flows. Recently, our three-dimensional code has been parallelized for high performance computing on massively parallel architectures [29]. The performance of the numerical technique has been benchmarked through various studies [6, 25-29].

\subsection{Dynamic contact model}

A complete mathematical description of the contact line motion including microlayer behavior is still a formidable task especially in three-dimensions. With a no-slip boundary condition at the wall, the shear stress becomes infinite near the contact line. Recently molecular dynamics simulations in the region of the contact point reveal several important physical aspects [16]. To prevent an infinite shear stress at the contact point, the interface must be allowed to slip along the wall. Interface slip has usually been modeled by the Navier-slip condition which displaces the contact line proportional to the shear stress at the wall. Recently, the Generalized Navier Boundary Condition (GNBC) was proposed [15] to include proper behavior of the contact line obtained from molecular theory. In addition to Navierslip, i.e. viscous stress on the wall, an unbalanced Young's stress is included to account for the interaction force acting on a very thin layer near the contact line. By considering the unbalanced Young's stress, slip information at the microscale can be modeled at the macroscale.

We followed a similar procedure proposed by Yamamoto et al. [15] to formulate the GNBC within the framework of the LCRM. We first allowed contact line movement proportional to shear stress at the contact point as in the Navier-slip model. The contact line velocity can be determined by the following equation: 


$$
U_{c l}=\left.\lambda \frac{\partial u}{\partial n}\right|_{\text {wall }}
$$

here, $U_{c l}$ denotes the contact line speed, $\partial u /\left.\partial n\right|_{\text {wall }}$ is the shear strain rate at the wall, and $\lambda$ is a proportionality slip constant usually the size of a grid cell. This slip constant would be a function of the distance from the contact line [30] but we assume it has a fixed value for simplicity in the current study. Henceforth, we will denote this constant as the "slip length". The Generalized Navier Boundary Condition (GNBC) additionally accounts for physical aspects of the contact line motion determined from molecular simulations describes interface slip as follows [15]:

$$
U_{c l}=\left.\lambda \frac{\partial u}{\partial n}\right|_{\text {wall }}+\lambda \frac{\tilde{\tau}^{\text {Young }}}{\mu}=\lambda\left\{\left.\frac{\partial u}{\partial n}\right|_{\text {wall }}+\frac{\sigma\left(\cos \theta_{s}-\cos \theta_{d}\right)}{\mu \Delta}\right\}
$$

Here, the first term on the right side represents viscous shear stress and the second term accounts for the unbalanced Young's stress. $\lambda$ is again a proportionality constant, i.e. slip length, and $\Delta$ is the size of a grid cell. $\theta_{s}$ represents the micro static contact angle and $\theta_{d}$ is the micro dynamic angle.

Since we track the interface, it is relatively straightforward to implement contact line dynamics in the LCRM. Fig. 2 illustrates the procedure for implementing the contact line model in the LCRM. First, we identify the contact point in 2D (see Fig. 2(a)) or contact line in 3D (see Fig. 2(b)). Here we explain our extended interface concept for the contact model in 2D. Using the interface normal (n $\left.\mathbf{n}_{f}\right)$ for the element contacting the wall and the normal $\left(\mathbf{n}_{s}\right)$ to the solid wall, we can identify the contact angles ( $\theta_{\text {contact }}$ in Fig. 2) associated with those contact points. We then extend the interface inside the wall with a specific angle $\left(\theta_{e x t}\right)$ to correctly impose the interfacial tension force in the governing equations as described below. The angle for this extension, i.e. $\theta_{\text {ext }}$ in Fig. 2, has been chosen to account for contact angle hysteresis. We use a simple hysteresis model as follows: 


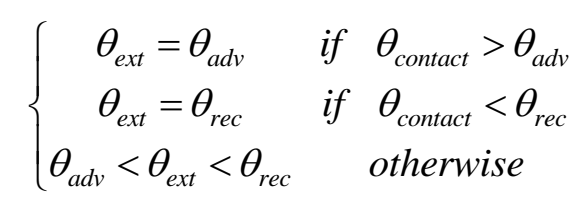

In our current study, constant advancing $\left(\theta_{a d v}\right)$ and receding $\left(\theta_{\text {rec }}\right)$ contact angles are used. The extension angle $\left(\theta_{\text {ext }}\right)$ is fixed to either the advancing $\left(\theta_{a d v}\right)$ or the receding $\left(\theta_{\text {rec }}\right)$ angle if the contact angle is greater than the advancing angle or less than the receding angle, respectively. Otherwise, the contact angle is free to move between the advancing and receding angles. More sophisticated models which relate contact line speed to the contact angle are available and could be incorporated in future work.

The force on a short segment of the front in $2 \mathrm{D}$ is given by:

$$
\delta F_{e}=\int_{\Delta s} \sigma \kappa \mathbf{n} \delta \mathrm{d} s
$$

We can rewrite Eq. (4) using the Frenet relation, $\kappa \mathbf{n}=d \mathbf{t} / d \mathbf{s}$ :

$$
\delta F=\int_{A}^{B} \sigma \frac{\partial \mathbf{t}}{\partial s} \delta \mathrm{d} s=\sigma\left(\mathbf{t}_{e x t}-\mathbf{t}_{f}\right) \delta
$$

For the extended surface, the tangential force is only computed on the side pointing away from the wall (see Fig. 2(a), $\vec{t}_{\text {ext }}$ ). By using the extended surface, the wall contacting element (containing $\vec{t}_{f}$ ) will have the correct opposing component $\left(\vec{t}_{\text {ext }}\right)$ of the matching interface tangential force thus automatically balancing the surface tension force in the direction of the solid normal $\left(\mathbf{n}_{s}\right)$. Moreover, Young's unbalanced force is accounted for naturally in this current extended surface formulation. The interfacial force balance in the direction tangential to the solid normal $\left(\mathbf{n}_{s}\right)$, on the segment near the wall including the extended surface, can be computed as follows: 


$$
\left(\delta F_{e} \cdot \mathbf{i}\right)_{\text {wall }}=\int \sigma \frac{\partial \mathbf{t}}{\partial s} \delta \mathrm{d} s \cdot \mathbf{i}=\sigma\left(\mathbf{t}_{\text {ext }}-\mathbf{t}_{f}\right) \delta \cdot \mathbf{i}=\sigma\left(\cos \theta_{\text {ext }}-\cos \theta_{\text {contact }}\right) \delta=\int_{\text {int }} \tilde{\tau}^{\text {Young }} \delta d y
$$

Here, $i$ represents the perpendicular direction to the wall and the last term indicates the integration of Young's unbalanced force to the $y$ (i.e. normal) direction near the wall. As can be seen from the figure, the extended surface will impose the unbalanced Young's stress [15] implicitly in the wall direction. Since a finite delta function was utilized to account for the surface force (see Eq. (14) (19)), its effect has been confined near the wall or the interface with a finite computational cell width of 4 cells along the interface. Thus, the extended surface accounts for the angle mismatch between $\theta_{\text {ext }}$ and $\theta_{\text {contact }}$ through increased curvature near the wall. This additional force will attribute the change of pressure during the projection of the velocity field (see Eq. (9) and Eq. (23) (27)). One more advantage of the extended surface is that the distance function computation from the interface can be naturally extended to the inner wall boundary which facilitates the computation of Eq. (10) near the solid wall. Thus, using the NBC (Navier Boundary Condition) will be sufficient to account for the correct interface slip (i.e. using Eq. (1)). As pointed out in Yamamoto et al. [15], most front capturing type methods compute curvature for the interfacial source term indirectly using a color function such as distance or volume fraction. In some case, iteration of the color function near the wall is necessary for imposing the correct contact condition which, however, increases computational time. With the extended surface, the interface condition at the contact line can be accounted for directly in the interfacial tension force term which will prove very effective when considering the full three-dimensional formulation.

Another point to note is that numerically we are computing an apparent contact angle (i.e. macroscopic angle) in the simulation. The exact force balance near the contact point should be computed using the actual microscopic contact angle. Recent molecular dynamics simulations of contact line behavior support Cox's relation [8] between macro and micro contact angle [15]:

$$
g\left(\theta_{\text {macro }}\right)=g\left(\theta_{\text {micro }}\right)+C a \ln \left(\frac{R}{l_{\text {slip }}}\right)+O(C a)
$$


Here $C a$ is the capillary number $(C a=\mu U / \sigma), R$ is the length scale, and $l_{\text {slip }}$ is the slip length. The functional form of $g(\theta)$ can be found in [14]. In the case where the second term on the right-hand side of Eq. (7) is sufficiently small, we can assume $\theta_{\text {micro }} \sim \theta_{\text {macro. }}$ In our benchmark simulation of drop impact on a wall, $R$ is of the order of $1 \mathrm{~mm}$ and $C a$ is around 0.014 for a water droplet with initial velocity of $1 \mathrm{~m} / \mathrm{s}$. If we assume the slip length $\left(l_{\text {slip }}\right)$ to be $1 \mathrm{~nm}$ as computed in $[15,17]$, the second term is of order 0.4 , less than unity. During the drop impact, the velocity of the contact line becomes much smaller than the initial impact velocity thus the second term would have a much smaller value. Therefore, we will confine our work to macroscopic modeling of contact line dynamics without considering the micro contact angle behavior.

The extended surface concept can be easily expanded to three-dimensions. As can be seen from Fig. 2(b), the basic process is the same as in 2D. First the contact line from the triangular element in contact with the wall (the element (1) (2) (3) in Fig. 2(b)) is identified. Then the contact line can be tagged (the line from the edge points (2) to (3)). Using the interface normal ( $\left.\mathbf{n}_{f}\right)$ of the wall contacting element and the solid normal ( $\left.\mathbf{n}_{s}\right)$, the contact angle can be computed. Again, the relation from Eq. (3) will convert contact angle to extension angle for contact angle hysteresis. In the 3D formulation, lines will be extended into the wall from points (2) and (3) to form points (A) and (B) (see Fig. 2(b)). These extended lines will be in the plane formed by the two normals, $\mathbf{n}_{f}$ and $\mathbf{n}_{s}$, respective to each point. For the continuous interface, the interface normal at the wall is computed using the distance function. The extended surface will initially form the rectangle (2) (3)(A)(B)). This will be broken into three triangular elements as in Fig. 2(B). This extension procedure is performed on all of the contact line elements. After the extension of the interface, advancement of the contact line is performed as in 2D using Eq. (1). The velocity gradient was computed using a first order approximation. The velocity in the normal direction from the wall at a distance of a grid cell was used to approximate the gradient. In the current simulation, simple solid structures such as a circle or cylinder were used where the distance from the solid can be obtained theoretically. Therefore the normal direction can be computed easily with the given solid distance function field even for complex solid structures. 


\subsection{Solution procedure}

The detailed method for solving the Navier-Stokes equations can be found in [6, 26-27]. Here we briefly describe the basic procedure. The governing equations for mass and momentum can be described in a single field form as follows:

$$
\begin{gathered}
\nabla \cdot \mathbf{u}=0 \\
\rho\left(\frac{\partial \mathbf{u}}{\partial t}+\mathbf{u} \cdot \nabla \mathbf{u}\right)=-\nabla P+\rho \mathbf{g}+\nabla \cdot \mu\left[(\nabla \mathbf{u})+(\nabla \mathbf{u})^{\mathrm{T}}\right]+\mathbf{F}
\end{gathered}
$$

where $\mathbf{u}$ is the velocity, $P$, the pressure and $\mathbf{g}$, the gravitational acceleration. A hybrid formulation for the surface tension force term, $\mathbf{F}$, is used to minimize parasitic currents:

$$
\mathbf{F}=\sigma \kappa_{H} \nabla I
$$

Here $\sigma$ represents the surface tension coefficient which is assumed to be constant in our current study. $I$ is the Heaviside function which has the value zero in one phase and one in the other. It is also used to describe material properties for density and viscosity,

$$
\begin{aligned}
& \rho=\rho_{L}+\left(\rho_{G}-\rho_{L}\right) I \\
& \mu=\mu_{L}+\left(\mu_{G}-\mu_{L}\right) I
\end{aligned}
$$

Here, the subscripts L and G stand for the liquid and gas phases, respectively.

We compute the hybrid form of the interface curvature field $\sigma \kappa H$ as [26]:

$$
\sigma \kappa_{H}=\frac{\mathbf{F}_{\mathrm{L}} \cdot \mathbf{G}}{\mathbf{G} \cdot \mathbf{G}}
$$


where

$$
\begin{gathered}
\mathbf{F}_{\mathrm{L}}=\int \sigma \kappa_{f} \mathbf{n}_{f} \delta\left(\mathbf{x}-\mathbf{x}_{f}\right) d A \\
\mathbf{G}=\int \mathbf{n}_{f} \delta\left(\mathbf{x}-\mathbf{x}_{f}\right) d A
\end{gathered}
$$

Here $\mathbf{x}_{f}$ is a parameterization of the interface and the geometric information, interface curvature, $\kappa f$, unit normal, $\mathbf{n} f$, and area of the interface element, $d A$ are computed directly from the Lagrangian interface. The discrete form of the expressions in Eq. (14) and (15) can be distributed onto the Eulerian grid by summing over interface elements, e, as follows:

$$
\mathbf{F}_{\mathrm{L} i, j}=\sum_{e} f_{e} D_{i, j}\left(x_{e}\right) \Delta A_{e}
$$

With $\Delta \mathrm{A}$ the element area and $D_{i, j}$ being a discrete approximation to the Dirac delta function, here in 2D:

$$
\mathrm{D}_{i j}\left(\mathbf{x}_{f}\right)=\frac{\delta\left(x_{f} / h_{x}-i\right) \delta\left(y_{f} / h_{y}-j\right)}{h_{x} h_{y}}
$$

where $h_{x}$ and $h_{y}$ are the dimensions of an Eulerian grid cell and

$$
\delta(r)= \begin{cases}\delta_{1}(r), & |r| \leq 1 \\ 1 / 2-\delta_{1}(2-|r|), & 1<|r|<2 \\ 0, & |r| \geq 2\end{cases}
$$

and 


$$
\delta_{1}(r)=\frac{3-2|r|+\sqrt{1+4|r|-4 r^{2}}}{8}
$$

For the axi-symmetric simulations, the circumferential component was added to Eq. (10)

$$
\mathbf{F}=\sigma \kappa_{H} \nabla I+\frac{\mathbf{n}_{r}}{r} \cdot i_{r}
$$

Here $\boldsymbol{n}_{r}$ represents the interface normal in the radial direction, $i_{r}$ is the unit vector in the $r$ direction, and $r$ is the radial position of the interface. The first term on the right-hand side can be computed similarly to Eq. (5) for plane 2D geometry (i.e. r-z plane) and the second term will account for the circumferential component of the curvature. For the three-dimensional case, equation (14) can be converted into a line integral around each interface element as:

$$
\mathbf{F}_{\mathrm{L}}=\int \sigma \mathbf{t} \times \mathbf{n} d s
$$

Here, $\mathbf{t}$ is a vector tangent to the edge of the element and $\mathbf{n}$ is a normal vector to the interface element. More detailed formation can be found in $[6,26,28]$

The interfacial elements are advected in Lagrangian fashion by integrating:

$$
\frac{d \mathbf{x}_{f}}{d t}=\mathbf{V}
$$

with a second order Runge-Kutta method where the interface velocity, $\mathbf{V}$, is interpolated from the Eulerian velocity. 
The Navier-Stokes equation, Eq. (9) is solved using a conventional projection method on a staggered grid. The discrete form of Eq. (9) can be written with lumping advection, diffusion, and gravitational terms into $\mathbf{A}$ as follows:

$$
\frac{\mathbf{u}^{n+1}-\mathbf{u}^{n}}{\Delta t}=\frac{1}{\rho^{n}}\left(\mathbf{A}^{n}+\mathbf{F}^{n}\right)-\frac{1}{\rho^{n}} \nabla_{h} P
$$

Here the subscript $h$ implies a spatially discrete operator. The convection term was discretized with a second order ENO method and central differences were used for the diffusion term. Time integration was performed with two substeps:

$$
\frac{\widetilde{\mathbf{u}}-\mathbf{u}^{n}}{\Delta t}=\frac{1}{\rho^{n}}\left(\mathbf{A}^{n}+\mathbf{F}^{n}\right)
$$

and

$$
\frac{\mathbf{u}^{n+1}-\tilde{\mathbf{u}}}{\Delta t}=-\frac{1}{\rho^{n}} \nabla_{h} P
$$

Here the variable $\tilde{\mathbf{u}}$ is the new fluid velocity while ignoring pressure. By enforcing $\mathbf{u}^{n+1}$ to be divergence free, the pressure can be found by solving

$$
\nabla_{h} \cdot\left(\frac{1}{\rho^{n}} \nabla_{h} P\right)=\frac{\nabla_{h} \cdot \tilde{\mathbf{u}}}{\Delta t}
$$

The updated velocity is

$$
\mathbf{u}^{n+1}=\tilde{\mathbf{u}}-\frac{\Delta t}{\rho^{n}} \nabla_{h} P
$$

A more detailed description of the solution procedure for such two-phase flows can be found in $[6,25-$ 28].

Recently, our interface tracking method, i.e. Level Contour Reconstruction Method, has been extended to fully three-dimensional simulations using a high performance, parallel code which we call BLUE on large-scale parallel computing architectures. BLUE can handle large property differences, where density and viscosity ratios are on the order of several thousand. Owing to the Level Set 
characteristics of the LCRM, parallelization is relatively straightforward. The program has been built on a modular basis thus application of the code to a wide variety of physical scenarios is possible. Various benchmarking tests including bubble rise, drop splash, and Faraday free surface instabilities were performed. Detailed parallel performance characteristics of the code can be found in [29]. In this work, the aforementioned contact modeling has been implemented in BLUE and tested for droplet impact on a stationary solid cylinder. 


\section{RESULTS AND DISCUSSIONS}

\subsection{Simulation settings and reference case}

We simulate a small water droplet impacting on a solid wall in order to study the detailed behavior of the contact line motion in a dynamic situation. The simulation conditions are essentially the same as those in Yokoi et al. [23]. The axisymmetric simulation geometry for the current test is shown in Fig. 3(a). A spherical droplet with initial radius $\mathrm{R}=1.14 \mathrm{~mm}$ is placed above the wall, emulating the impact condition. The initial impact velocity is $1 \mathrm{~m} / \mathrm{s}$. The box size in both the horizontal and vertical directions is chosen to be $5 \mathrm{~mm} \times 5 \mathrm{~mm}$. Open conditions are imposed on the side and top boundaries. Water and air properties at atmospheric pressure are chosen for the current study. The density and viscosity of the water are $1000 \mathrm{~kg} / \mathrm{m}_{3}$ and $1.0 \times 10_{-3} \mathrm{~N} \cdot \mathrm{s} / \mathrm{m}_{2}$, respectively. Those for air are $1.25 \mathrm{~kg} / \mathrm{m}_{3}$ and $1.82 \times 10_{-5} \mathrm{~N} \cdot \mathrm{s} / \mathrm{m}_{2}$ respectively. The surface tension coefficient is $0.072 \mathrm{~N} / \mathrm{m}$.

Fig. 3(b) shows the contact angle variation with contact line speed according to Yokoi et al. [23]. Red squares represent experimental measurements and the blue dotted line is the curve fitted profile using the following relation:

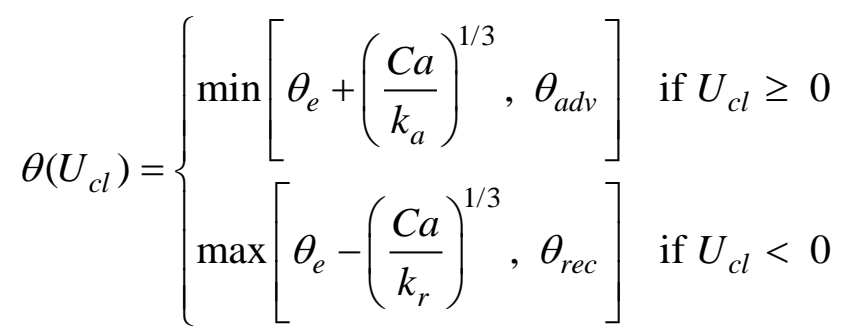

Here $\theta_{e}$ is the equilibrium contact angle. Yokoi et al. [23] recommend $\theta_{a d v}$ and $\theta_{\text {rec }}$ values of $114_{\mathrm{o}}$ and 52 ond $k a$ and $k r$ values of $9 \times 10-9$ and $9 \times 10-8$, respectively. Since, our current treatment of the contact line assumes constant advancing and receding angles, we choose to use an intermediate value of 65 o for the receding angle when a somewhat large variation is present. For the advancing angle, we use 114, the same as Yokoi's condition.

Figure 4 shows the contact diameter variation during the impact process. A $256 \times 256$ grid is used 
for this reference case. A non-dimensional slip ratio $(\lambda / \Delta x)$ of 0.5 is used for the Navier-slip condition based on Eq. (1). The Navier-slip condition was applied only to the interface movement not the fluid flow near the wall (i.e. interface slip which will be introduced in section 3.2). A time step size of 10-6 was used which is below the capillary time step constraint from Brackbill et al. [31] ( $\Delta$ tcapillary $=$ $\left.\sqrt{\left(\rho_{L}+\rho_{G}\right) \Delta x^{3} /(4 \pi \sigma)} \sim 3 \times 10^{-6}\right)$. We also considered a time step limit based on restricting the movement of the interface to less than 0.1 times the grid cell size during a time step. As can be seen in the figure, the contact diameter calculated from the current formulation matches very well with the experimental measurements from Yokoi et al. [23]. For further detailed analysis, we divide the simulation results for contact diameter into 6 different regions (see alternate long and short dashed lines in Fig. 4). The first region (I) is the initial spreading of the droplet at largest impact velocity. We also plot the interface evolution including the extended surface at the contact line to show the behavior of the dynamic contact angle in Fig. 5. Fig. 5 (b) and (c) depict the interface evolution during phase I. The contact angle is fixed to the advancing angle and we can notice that a rim builds up around the edge of the droplet. During phase II, the change in contact diameter with time decreases to zero. Fig.5 (d) shows the typical interface profile during phase II. The rim at the edge of the droplet bulges slightly upward then starts to be drawn into the center. The contact point is almost pinned to the same location during this transition period. The interface then contracts rapidly inward and pushes the fluid to the center creating an upward motion (phase III). Fig. 5(e) shows that the contact angle now approaches the fixed receding angle. After a linear decline of the contact diameter in phase III, the contact point is pinned to a fixed location again (phase IV). During phase IV, upward motion at the center is reduced due to the increased downward gravitational force which depresses the droplet and pushes the contact line slightly outward (Phase V). After this, there is insufficient force to move the contact line and the contact diameter remains nearly constant (Phase VI). In our contact model formulation, the contact angle in Phase VI, the static contact angle, is not prescribed thus it will be determined by the fluid motion and interface interaction with a value between the advancing and receding angles. More sophisticated modeling is possible and can readily be implemented with few modifications. However, as mentioned earlier, we decided to use a simpler form considering the extension to complex 3D flows. 
The pressure field and velocity vectors during Phases I to V are shown in Figs. 6 and 7. Initially the higher pressure inside the droplet due to curvature radiates to the outer rim (Fig. 6 (a)). We also note a strong counter-clockwise vortex near the contact line (Fig. 7(a)). With spreading of the interface, high pressure builds up at the far end of the droplet (Fig. 6(b)) then recoils to the center (Fig. 6(c)). The counter-clockwise vortex moves slightly inward with decreasing magnitude (Fig. 7(b) and (c)). High pressure near the contact line pushes the outer rim up and inward. Pressure increases at the center of the droplet (Fig. 6(d)) and pushes the interface upward (Fig. 6(e)). During the receding phase, the vortex rotates clock-wise with increasing magnitude (Fig. 7(d)). The pressure increases slightly at the top of the droplet, then gravity pulls the interface downward (Fig. 6(f) and Fig. 7(f))

\section{2 slip model test}

As discussed in section 2, our contact line model uses the Generalized Navier Boundary Condition (GNBC). The contact line motion is proportional to the velocity gradient at the wall. The nondimensional slip ratio, i.e. slip length divided by grid size, is computed using interpolated values of the ghost velocity field inside the wall (See Fig. 2 (a)). In other work [12, 14, 23] the actual velocity is used for computing slip along the entire wall. Another approach is to allow some relaxation, where the slip condition is applied only near the contact line (usually over 3 to 4 grid cells to be consistent with the support of the discretized Dirac kernel in the Front-Tracking approach). Since our interface method tracks both the Lagrangian interface as well as the Eulerian distance function, we can accommodate how the slip boundary condition is computed. We denote "momentum slip" to indicate that the specified slip length or slip ratio is applied to the actual velocity field near the wall and "interface slip" to indicate that the specified slip length or slip ratio is only applied for interface movement by adjusting the ghost velocity at the wall.

Fig. 8 shows the contact diameter evolution for momentum slip (Fig. 8(a)) and interface slip (Fig. $8(\mathrm{~b}))$ for a variety of non-dimensional slip ratio $(\lambda / \Delta x)$. Here $\lambda$ is the proportionality constant as shown in Eq. (1) and $\Delta x$ is the chosen grid size. As can be seen in the figure, momentum slip results are very sensitive to the slip ratio. With the highest slip ratio of 0.75 , the drop spreads beyond the extent of the 
domain in the $x$ direction. With interface slip, the slip ratio effect is relatively minor compared to the momentum slip model. There is only a slight increase of maximum spread diameter with increasing slip ratio. The velocity of the contact line $\left(U_{c l}\right)$ is also plotted in Fig. 9 corresponding to the cases in Fig. 8. With higher slip ratio, the momentum slip model shows large changes in contact line velocity. On the other hand, the interface slip model shows only a small difference. Initially, during phase I, the contact velocity decreases rapidly then changes sign in phase II where retraction begins. The contact velocity further decreases to a minimum value then rises. In phase IV the contact velocity is nearly constant. The most distinctive difference can be noticed in phase V. With larger slip ratio, the velocity bump in phase $\mathrm{V}$ is slightly retarded and also slightly higher in magnitude. To investigate the effect of the two slip models more closely, we compare the shear stress distribution during phase I where the largest shear stress can be expected. Fig. 10 plots the shear stress at the wall for both the full and interface slip models with a slip ratio of 0.5 at a time of $1 \mathrm{~ms}$. As can be seen in the figure, the momentum slip model shows a much smaller shear stress distribution compared to the interface slip model. In both the full and interface slip models, the contact diameter and contact line speed approach similar values as the slip ratio decreases. This indicates that a small slip ratio should be used for the momentum slip model to obtain an accurate solution. It is also possible to use partial slip however our intention was to devise a simple and general procedure for full three-dimensional simulations. Thus, we choose interface slip as our operational model for contact line motion.

\subsection{Grid convergence test with energy analysis}

Before full simulation techniques for two-phase flow with dynamic contact models became available, drop impact processes were studied through an energy analysis assuming simple drop shapes. Attante et al. [32] used spherical cap, cylinder, or rimmed cylinder geometries to emulate the droplet during the spreading process. They identified kinetic, potential (surface and gravitational), and dissipation energy terms for the spreading droplet. For the viscous energy term, film, line, and viscous dissipation were considered based on de Gennes [33] work. They ignored the film dissipation term due to partial wetting of the droplet spreading and line dissipation was also neglected in their analysis. 
Recently, Bange and Bhardwaj [34] investigated conversion of the energy terms during droplet impact using a FEM model.

To gain a more detailed physical understanding, we analyze the drop impact process in our simulations using an energy analysis similar to that of Attante et al. [32], where we consider surface, gravitational, potential, kinetic, and dissipation energy changes. Since the droplet impact in our case is for partial wetting, we neglect the film dissipation term. However, we include line dissipation attributed to motion of fluid molecules at the contact line. The total energy variation can be cast into the following form:

$$
\Delta E_{\text {total }}=\Delta E_{\text {surface }}+\Delta E_{\text {kinetic }}+\Delta E_{\text {potential }}+\Delta E_{\text {viscous }}+\Delta E_{\text {cld }}=0
$$

Here $\Delta E_{\text {surface }}$ represents surface energy released by droplet impact, $\Delta E_{\text {kinetic }}$ is the total kinetic energy, $\Delta E_{\text {viscous }}$ is the viscous dissipation, $\Delta E_{\text {potential }}$ is the gravitational energy, and $\Delta E_{\text {cld }}$ is the contact line dissipation which is the energy necessary to overcome contact line movement. Each energy term can be computed as:

$$
\begin{gathered}
\Delta E_{\text {sufface }}=\sigma_{l v} \Delta A_{l v}+\sigma_{l s} \Delta A_{l s}+\sigma_{s v} \Delta A_{s v} \\
\Delta E_{\text {kinetic }}=\int_{V_{d}} \frac{1}{2} \rho\left\{\left(u^{2}+v^{2}+w^{2}\right)-\left(u_{0}^{2}+v_{0}^{2}+w_{0}^{2}\right)\right\} d v \\
\Delta E_{\text {potential }}=\rho V_{d} g\left(z_{c}-z_{c_{0}}\right) \\
\Delta E_{\text {viscous }}=\int_{0}^{t}\left(\int_{V_{d}} \Phi d v\right) d t
\end{gathered}
$$

with

$$
\begin{gathered}
\Phi=2 \mu\left[\left(\frac{\partial u}{\partial x}\right)^{2}+\left(\frac{\partial v}{\partial y}\right)^{2}+\left(\frac{\partial w}{\partial z}\right)^{2}+\frac{1}{2}\left\{\left(\frac{\partial v}{\partial x}+\frac{\partial u}{\partial y}\right)^{2}+\left(\frac{\partial w}{\partial y}+\frac{\partial v}{\partial z}\right)^{2}+\left(\frac{\partial u}{\partial z}+\frac{\partial w}{\partial x}\right)^{2}\right\}\right] \\
\Delta E_{c l d}=\int_{0}^{t} \beta\left(2 \pi R\left[\frac{d R}{d t}\right]^{2}\right) d t
\end{gathered}
$$


Here $V_{d}$ and $d v$ represent the volume of the droplet and a local infinitesimal element, respectively. $z_{c}$ is the centroid of the droplet and the subscript 0 represents the initial state. $\Delta A(=A-A 0)$ is the change in the interfacial area between solid $(s)$, vapor $(v)$, and liquid $(l)$ phases. $R$ in Eq. (20) represents the contact radius at the wall and $\beta$ is a friction coefficient. The energy variation over time can be computed throughout the simulation except for the $\Delta E_{c l d}$ term since the friction efficient $(\beta)$ is unknown. Since the total energy should be conserved as in Eq. (29), the change of the energy in contact line $\left(\Delta E_{c l d}\right)$ has been computed from other energy terms, Eq. (30), (31), (32), and (33) which can be calculated directly from the simulation. Considering energy conservation during the impact process, we can obtain the energy required to overcome contact line movement, i.e. $\Delta E_{c l d}$, as follows:

$$
\Delta E_{\text {cld }}=-\left(\Delta E_{\text {surface }}+\Delta E_{\text {kinetic }}+\Delta E_{\text {potential }}+\Delta E_{\text {viscous }}\right)
$$

Since the contact radius during impact can be computed, the friction coefficient can be obtained using Eqs. (34) and (35). The contact radius, R, can be tracked by storing the contact point throughout the simulation. The derivative of the contact radius over time can also be computed using the time history of the stored R.

We plot the variations of each energy term during the impact process for the reference case (shown in Fig. 4) in Fig. 11. As can be seen from the figure, viscous dissipation increases to a saturated value while kinetic energy decreases with slight oscillations which are reflected in the oscillation of surface energy. Potential energy variation is almost negligible for this small droplet. The computed $\Delta E_{\text {cld }}$ term is depicted as the solid red line. The energy required to overcome contact line movement increases and it is interesting to note that its value is comparable to viscous dissipation and cannot be ignored.

For the interface slip model, we also conduct grid convergence tests to determine how the energy balances depend on resolution. As pointed out in [15], simulations using the GNBC dynamic contact model for a given choice of slip length show grid resolution dependence. That is, it is the slip ratio, $\lambda / \Delta x$, that should be fixed for different grid resolutions and not the slip length, $\lambda$. We have previously 
defined $\lambda$ as the actual slip length and $\lambda / \Delta x$ as the slip ratio which depends on grid size $(\Delta x)$. In the energy analysis, we fix either the slip ratio $(\lambda / \Delta x)$ or the slip length $(\lambda)$ to demonstrate the difference in convergence characteristics. We also compare energy balances for the momentum slip and interface slip conditions.

Figure 12 shows the change in kinetic energy for different slip models. The top row is for interface slip and the bottom row is for momentum slip. The right-hand column uses a constant slip ratio condition and the left-hand column is for constant slip length. The slip models show convergence with increasing grid resolution except for the momentum slip model with constant slip length where, under these conditions, the highest grid resolution solution tends to diverge. Thus, in order to provide comparisons, we reduce by half the slip ratio and slip length for the momentum slip model compared to the interface slip cases. In Fig. 13, we plot the evolution of surface energy for the different slip models. In the case of momentum slip with constant slip length, Fig. 13(d), the surface energy at the highest resolution diverges. With increased resolution, the wall shear stress near the contact line increases and results in unphysical motion of the contact line. Similar behavior can be noticed in Fig. 13(b) where interface slip with constant slip length is used. The solution does not converge with increased grid resolution. On the other hand, with a fixed slip ratio, the evolution of the surface energy shows convergence with grid resolution.

Figure 14 depicts the energy change due to viscous dissipation. Except for the momentum slip model with constant slip length, the viscous dissipation increases with higher resolution. The momentum slip condition has a lower viscous dissipation compared to the interface slip condition as was seen in Fig. 10. The wall shear stress for both the interface and the momentum slip condition at different resolutions is plotted in Fig. 15 at a time of 1 ms during phase I as in Fig. 10. Again, the shear stress at the wall is much smaller for the momentum slip condition. With increased resolution, a sharp peak of the shear stress is generated around the contact line as already described in $[14,15]$. However, the shear stress distribution becomes similar away from the contact line with increased mesh resolution.

Figure 16 shows the computed friction coefficient for the different slip models based on Eqs. (34) and (35). For all the models, the friction coefficient essentially decreases with increased grid resolution. 
The friction coefficient for the momentum slip model with constant slip length decreases in an unpredictable way with higher resolution. The interface slip model with constant slip length and the momentum slip model with constant slip ratio show almost a linear decrease of the friction coefficient with sufficiently high resolution. The friction coefficient for the interface slip model with constant slip ratio shows a relatively narrow variation with different resolution compared to the other cases.

The evolution of the contact diameter for different slip models is shown in Fig. 17. The interface slip model with constant slip length and the momentum slip model with constant slip ratio both generate a very similar variation of the contact diameter with varying grid resolution but do not exhibit convergence with increased resolution. The momentum slip model with constant slip length shows a very large deviation of the contact diameter at the highest resolution as can be expected from the behavior of the energy changes ((d) of Figs. 12, 13, 14). As can be deduced from the behavior of the energy changes associated with the different slip models, the interface slip model with constant slip ratio shows the best convergence characteristics. In this case, the lowest grid resolution has the largest error but quickly converges with higher grid resolution. In our current two-phase numerical model, the interfacial source term in the momentum equation (Eq. (9)) is a Dirac delta distributed over nearby grid cells (usually two grid cells in each direction) thus generating a resolution dependent distribution. The physical region affected by the interface source term narrows with increased resolution. Thus, maintaining a constant slip ratio, i.e. decreasing slip length, with increased resolution would be mathematically appropriate as indicated by Yamamoto et al. [15]. Considering these convergence characteristics, it seems that the best choice would be to keep the slip ratio constant for different grid resolutions.

We also tested the effect of slip ratio on the variation of energy changes for the interface slip model with 2562 resolution in Fig. 18. The kinetic energy variation is almost identical for different slip ratios and there are only slight changes in the viscous dissipation and surface energy terms. On the other hand, the friction coefficient $(\beta)$ has noticeable differences. With decreased slip ratio, the friction coefficient increases. Considering Fig. 16(a), we believe that the slip ratio should decrease in order to generate a converged solution for the friction coefficient. With increasing grid resolution, near wall regions could better capture the detailed physics of the microlayer thus the slip length should approach the physical 
value which is known to be very small.

In Fig. 19, we show the contact angle distribution dependence on contact line velocity with different slip ratios. Different slip ratios ranging from 1.5 to 0.125 have been considered. As can be seen in the figure, with larger slip ratio, the contact angle has a sharper transition from the receding to advancing angle. As we decrease the slip ratio, the contact angle tends to deviate from the constant input values for the advancing (114o) and receding (65o) angles. The deviation becomes larger with decreasing slip ratio. We can see that the contact angle profile in the receding region can be approximated well using a slip ratio of 0.125 . With a high enough slip ratio, the contact angle shows a very similar trend, a sharp change to a fixed input value. Similar behavior was shown in Yamamoto et al. [15]. In their simulation of meniscus rise, the advancing contact angle linearly increased with an increase of contact line velocity. We can generate a similar trend by changing the slip ratio with our current contact modeling. As pointed out by Yamamoto et al. [15], the slip ratio can be considered to represent surface characteristics of the microscale surface structure such as surface roughness.

\subsection{Effect of contact angles}

We compare the effect on contact diameter for differing advancing and receding contact angles. For the advancing contact angle, we vary its value from 94 o to 134 o in increments of 10 . For the receding contact angle, we vary its value from 55 o to 75 o in increments of 5 o. Thus, a total of five cases are compared for advancing angles and five for receding angles. Fig. 20 shows the contact diameter dependence on the advancing contact angles. As can be seen in the figure, the advancing angle affects the maximum spreading radius considerably. The interface initially spreads to its maximum with high impact velocity during phase II then spreads again with a relatively small spread in phase VI. The advancing angle affects this spreading behavior significantly. In Fig. 21, the changes of kinetic, viscous, and surface energy are plotted for different advancing angles. The computed friction coefficient is also shown in Fig. 21(d). The changes of kinetic energy are relatively minor compared to the viscous and surface energies. Initially, the kinetic energy decreases almost identically for different advancing angles. At the end of phase I, the lower advancing angle shows a slightly delayed response. With lower 
advancing angle, the viscous dissipation and surface energy increase while the friction coefficient increases with increasing advancing angle.

The effect of varying the receding angle is shown in Fig. 22. Contrary to the advancing angle (Fig. 20), the receding angle affects the drop retraction motion considerably in phase IV and V. With increasing receding angle (i.e. the interface can slip more freely), the interface retracts much further to a smaller contact diameter value. It is interesting to note that the retracting interface spreads again to a similar contact diameter. The energy variation dependence on different receding angles is shown in Fig. 23. In this case, kinetic energy and viscous dissipation are quite similar for all of the receding angles. The most significant discrepancy can be noticed in the surface energy change after phase III (during the retraction phase). With increased receding angle, the friction coefficient decreases but the surface energy change increases during the retraction phase.

Considering the effect of contact angle and slip ratio, we now adjust the reference case to obtain a more accurate comparison with experimental data. To match the contact angle variation vs contact line speed, we use a slip ratio of 0.125 for the receding phase while keeping a value of 0.5 for the advancing phase. The computed contact diameter is shown in Fig. 24 along with the plot of contact angle variation vs contact line speed. With this modified slip condition, the contact line diameter somewhat better represents experimental values especially in the final phase. Using different slip ratios for the advancing and receding phases, we can model contact angle variations which are very close to the experiment.

\subsection{D Droplet impact on a cylinder}

To ascertain the applicability and performance of our contact line formulation in 3D, we simulate a droplet impacting on a cylindrical solid surface. Droplet impact on cylindrical surfaces is a very important phenomenon in many processes such as that by which droplets pass through a porous structure $[35,36]$. Several studies have been performed to identify the mass of the droplet which remains on the solid after impact [36] or the impact of a droplet on a flexible wire [37], however very few references are available. Here, we simulate droplet impact on a cylindrical solid structure to demonstrate the capability of our current contact model for challenging three-dimensional problems. The presence of 
complex solid structures is accounted for by using the ghost fluid method which satisfies the momentum equation by adjusting the ghost node value inside the wall [40]. In this study, we focus on a qualitative comparison of impact behavior with different contact angles to assess the validity of our contact model in general 3D simulations.

Details of the simulation geometry are described in Fig. 25. The simulation domain in these simulations is $8 \mathrm{~mm} \times 8 \mathrm{~mm} \times 16 \mathrm{~mm}$. The code is fully parallelized for distributed processing architectures and we divide the simulation domain into 32 subdomains. Each local subdomain has a $64 \times 64 \times 32$ grid for a total resolution of $128 \times 128 \times 256$ grid cells. The total simulation time was approximately 1 day on a 32 core CPU with $2.6 \mathrm{GHz}$ processors. A solid cylinder $3 \mathrm{~mm}$ in diameter is placed $9 \mathrm{~mm}$ from the bottom wall. A spherical droplet $2.7 \mathrm{~mm}$ in diameter is placed above the cylinder and impacts it with a velocity of $0.75 \mathrm{~m} / \mathrm{s}$. The physical properties are the same as the previous case for drop impact on a flat wall. Three different cases are tested. The first uses identical advancing and receding angles as in the previous impact problem $\left(\theta_{a d v}=114 \mathrm{o}, \theta_{r e c}=65 \mathrm{o}\right)$. For the second test, we use a hydrophilic surface which emulates the condition of a water droplet hitting a glass surface. For this hydrophilic condition, we use an advancing angle of $60^{\circ}$ and a receding angle of 34 . The last case has a highly hydrophobic surface with an advancing angle of $165^{\circ}$ and a receding angle of 160 . Such superhydrophobic nanostructured surfaces can now be engineered [38].

The interface evolution for each test case is shown in Fig. 26 (regular case), Fig. 27 (hydrophilic case) and Fig. 28 (super-hydrophobic case). As can be seen in Fig. 27, the hydrophilic surface tends to slide around the cylinder during impact and cover a wider area of the cylinder. The spreading rim of the water droplet slides around the cylindrical surface and merges underneath the cylinder to form a hanging droplet. With larger advancing and receding angles (regular case Fig. 26), the spreading motion of the droplet at the impact becomes considerably restricted compared to the hydrophilic surface. For this case, the spreading rim in the axial direction of the cylinder retracts more quickly than the rim around the cylinder. An elongated half ring shaped drop continues to retract along the cylinder and ultimately forms a static droplet sitting on top of the cylinder. For the hydrophobic surface (Fig. 28), we can observe completely different interface dynamics. The droplet cannot spread enough to wet the 
cylindrical surface and retracts very quickly in the axial direction of the cylinder. With this increased retraction speed of the rim, the half ring shaped drop detaches from the cylinder surface. The elongated droplet oscillates to become a spherical drop separated from the cylinder. Our current contact angle formulation shows promise in treating general contact line behavior on complex solid structures. A more detailed quantitative analysis of droplet impact on a cylindrical surface is necessary and will be conducted in future work. 


\section{CONCLUSION}

We formulate a simple contact line model in the context of a Front Tracking based method for simulation of multiphase flows. Our main objective was to devise an efficient and accurate method which is also readily applicable to complex three-dimensional simulations. Special attention was paid to the dynamic contact angle model where large variations in advancing and receding angles are expected. The advancing and receding contact angles are imposed with the aid of an extended interface from the contact point (contact line in 3D) into the solid. The angles for the interface elements in the extended surface are defined according to the values of the contact angle for the fluid side interface elements. If the computed contact angle for the fluid side Lagrangian elements which contact the wall is larger than the given advancing angle, the extended surface is given the value of the advancing angle and analogously for the receding angle. This procedure naturally applies the Generalized Navier Boundary Condition (GNBC). The interface contacting the wall must slip in order to prevent infinite shear stress near the contact point. We used a conventional Navier-Slip condition. Since the Lagrangian elements are explicitly tracked, we are able to apply the slip condition locally only on the interface motion and not the full fluid velocity field. Ghost values of the velocity in the solid boundary which satisfy the given slip ratio are used to advect the interface along the wall. Results for contact diameter and contact line speed are much less sensitive to the slip ratio when the local slip condition is applied as opposed to imposing momentum slip along the wall.

In order to study the detailed dynamics of the drop impact process, we investigated the time evolution of changes to surface, kinetic and potential energies as well as both viscous and contact line dissipation. The energy needed to overcome contact line movement was computed using these other calculated energy terms. The changes for each energy term as well as the friction coefficient are compared for various grid resolutions and show that the interface slip model demonstrates good grid convergence compared to the momentum slip model. In addition, we show that results for an imposed constant slip length do not converge correctly with increased resolution. The detailed behavior for cases of imposed slip ratio or slip length was compared and suggest that the slip ratio model performs better. 
The effect of varying the advancing and receding angles on droplet impact was also investigated. The advancing contact angle affects the spreading radius considerably while the receding angle effect is significant only in the drop impact retraction phase. Finally, we simulated droplet impact using different slip ratios for the spreading and retracting phases which match experimental results more accurately, especially when considering contact angle variation vs contact line speed.

Our formulation extends in a straightforward way to three-dimensional simulations which we demonstrate for the case of droplet impact on a solid cylinder. Three impact scenarios with different contact angles were simulated to model hydrophilic, regular, and super-hydrophobic behaviors. Both the hydrophilic and regular cases wet the solid cylinder while the super-hydrophobic surface shows completely different characteristics during impact. We conclude that this simple contact line formulation can simulate realistic contact line dynamics in two- and three-dimensions with proper approximation of contact angles and slip ratio. 


\section{ACKNOWLEDGEMENTS}

This work is supported by (1) the Basic Science Research Program through the National Research Foundation of Korea (NRF) funded by the Ministry of Science, ICT and future planning (NRF2014R1A2A1A11051346) and (2) by computing time at the Institut du Developpement et des Ressources en Informatique Scientifique (IDRIS) of the Centre National de la Recherche Scientifique (CNRS), coordinated by GENCI (Grand Equipement National de Calcul Intensif) grant 2016 A0022B06721. 


\section{REFERENCES}

1. Boreyko J. B. and Chen C.H., Self-Propelled Dropwise Condensate on Superhydrophobic Surfaces, Physical Review Letters, 103, 184501 (2009).

2. Hirt C.W., Nichols B.D., Volume of fluid (VOF) method for the dynamics of free boundaries, $J$. Comput. Phys. (1981), 39, 201-225.

3. Osher S., Fedkiw R.P., Level Set Methods: an overview and some recent results, J. Comp. Phys. (2001), 169, 463-502.

4. Jamet D., Lebaigue O., Coutris N., Delhaye J. M., The second gradient method for the direct numerical simulation of liquid-vapor flows with phase-change, J. Comput. Phys. (2001), 169, 624651.

5. Tryggvason G., Scardovelli R., Zaleski S., Direct Numerical Simulations of Gas-Liquid Multiphase Flows, Cambridge University Press, Cambridge, England (2011).

6. Shin S., Juric D., Modeling three-dimensional multiphase flow using a level contour reconstruction method for front tracking without connectivity, J. Comput. Phys., 180, 427-470 (2002).

7. Hocking L.M., Rivers A.D., The spreading of a drop by capillary action, J. Fluid Mech., 121, 425442 (1982).

8. Cox R.G., The dynamics of the spreading of liquids on a solid surface. Part 1. Viscous flow, $J$. Fluid Mech., 168, 169-194 (1986).

9. Blake T. D., The physics of moving wetting lines, J. Colloid Interface Sci., 299, 1-13 (2006).

10. Huang H., Liang D., Wetton B., Computation of a moving drop/bubble on a slid surface using a front-tracking method, Comm. Math. Sci., 2(4), 535-552 (2004).

11. Ren W., E W., Boundary conditions for the moving contact line problem, Phys. Fluids, 19, 022101 (2007).

12. Afkhami S., Zaleski S., Bussmann, M., A mesh-dependent model for applying dynamic contact angles to VOF simulations, J. Comput. Phys., 228,5370-5389 (2009). 
13. Dupont J., Legendre D., Numerical simulation of static and sliding drop with contact angle hysteresis, J. Comput. Phys., 229, 2453-2478 (2010).

14. Sui Y., Spelt P. D.M., An efficient computational model for macroscale simulations of moving contact lines, J. Comput. Phys., 242, 37-52 (2013).

15. Yamamoto Y., Ito T., Wakimoto T., Katoh K., Numerical simulations of spontaneous capillary rises with very low capillary numbers using a front tracking method combined with generalized Navier boundary condition, Int. J. Multiphase Flow, 51, 22-32 (2013).

16. Qian T., Wang X., Sheng P., Molecular scale contact line hydrodynamics of immiscible flows, Physical Review E, 68, 016306 (2003).

17. Yamamoto Y., Tokeida K., Wakimoto T., Ito T., Katoh K., Modeling of the dynamic wetting behavior in a capillary tube considering the macroscopic-microscopic contact angle relation and generalized Navier boundary condition, Int. J. Multiphase Flow, 59, 106-112 (2014).

18. Zhang Q., Qian T., Wang X., Phase field simulation of a droplet impacting a solid wall, Phys. Fluids, 28, 022103 (2016).

19. Bussmann M., Mostaghimi J., Chandra S., On a three-dimensional volume tracking model of droplet impact, Phys. Fluids, 11, 1406-1417 (1999).

20. Sikalo S., Wilhelm H. D., Roisman I. V., Jakirlic S., Tropea C., Dynamic contact angle of spreading droplets: experiments and simulations, Phys. Fluids, 17, 062103 (2005).

21. Gunjal P.R., Ranade V.V., Chaudhari R.V., Dynamics of drop impact on solid surface: experiments and VOF simulations, AIChE Journal, 51, 59-78 (2005).

22. Fukai J., Zhao Z., Poulikakkos D., Megaridis C.M., Miyatake O., Modeling of the deformation of a liquid droplet impinging upon a flat surface, Phys. Fluids, 5, 2588-2599 (1993).

23. Yokoi K., Vadillo D., Hinch J., Hutchings I., Numerical studies of the influence of the dynamic contact angle on a droplet impacting on a dry surface, Phys. Fluids, 21, 072102 (2009).

24. Tanner L.H., The spreading of silicone oil drops on horizontal surfaces, J. Phys. D: Appl. Phys., 12, 1473-1484 (1979).

25. Shin S., Juric D., Simulation of droplet impact on a solid surface using the level contour reconstruction method, Journal of Mechanical Science and Technology, 23, 2434-2443 (2009). 
26. Shin S., Juric D., A hybrid interface method for three-dimensional multiphase flows based on fronttracking and level set techniques, Int. J. Num. Meth. Fluids, 60, 753-778 (2009).

27. Shin S., Juric D., High order level contour reconstruction method, Journal of Mechanical Science and Technology, 21(2), 311-326 (2007).

28. Shin S., Abdel-Khalik S. I., Daru V., Juric D., Accurate representation of surface tension using the level contour reconstruction method, J. Comput. Phys., 203, 493-516 (2005).

29. Shin S., Chergui J., Juric D., A solver for massively parallel direct numerical simulation of threedimensional multiphase flows, Journal of Mechanical Science and Technology, 31(4), 1739-1751 (2017).

30. Kirkinis E., Davis S.H., Hydrodynamic theory of liquid slippage on a solid substrate near a moving contactline, Physical Review Letters, 110, 234503 (2013).

31. D. Fabian and G.M. van Wachem Berend, Numerical time-step restrictions as a result of capillary waves, J. Comp. Phys., 285, 24-40 (2015).

32. Attante P., Girard F., Morin V., An energy balance approach of the dynamics of drop impact on a solid surface, Physics of Fluids, 19, 012101 (2007).

33. Gennes de P. G., Wetting: statics and dynamics, Rev. Mod. Phys., 57(3), 827-863 (1985).

34. Bange P.G., Bhardwaj R., Computational study of bouncing and non-bouncing droplets impacting on superhydrophobic surfaces, Theor. Comput. Fluid Dyn., 1-25 (2015).

35. Li Q., Chai Z., Shi B., Liang H., Deformation and breakup of a liquid droplet past a solid circular cylinder: A lattice Boltzmann study, Physical Review E, 90, 043015 (2014).

36. Hung L.S., Yao S.C., Dripping phenomena of water droplets impacted on horizontal wire screens, Int. J. Multiphase Flow, 28, 93-104 (2002).

37. Comtet J., Keshavarz B., Bush J.W.M., Drop impact and capture on a thin flexible fiber, Soft Matter, $12,149-156(2016)$.

38. Nam Y., Kim H., Shin S., Energy and hydrodynamic analyses of coalescence-induced jumping droplets, Applied Physics Letters, 103, 161601 (2013).

39. Yamamoto, Y., Higashida, S., Tanaka, H., Wakimoto, T., and Ito, K, Numerical analysis of contact line dynamics passing over a single wettable defect on a wall, Phy. Fluids, 28, 082109 (2016) 
40. Shin, S. and Abdel-Khalik, S. I., Numerical modeling of evaporating thin liquid film instability on a heated cylindrical rod with parallel and cross vapor flow, Nuclear Science and Engineering, 156, $1-16(2007)$ 


\section{FIGURE CAPTIONS}

Fig. 1 General description of the LCRM.

Fig. 2 Description of the present contact model with extended surface for (a) two-dimensional (b) three-dimensional simulation.

Fig. 3 Numerical setup for benchmark tests (a) simulation geometry (b) contact line speed vs. contact angle from experimental measurements.

Fig. 4 Evolution of the contact diameter with time for the reference case.

Fig. 5 Interface evolution with time including contact angle variation throughout the simulation.

Fig. 6 Pressure distribution for the reference case.

Fig. 7 Velocity vector distribution for the reference case.

Fig. 8 Comparison of momentum slip (a) and interface slip (b) models for contact diameter variation with different slip ratios.

Fig. 9 Comparison of momentum slip (a) and interface slip (b) models for contact line velocity with different slip ratios.

Fig. 10 Wall shear stress distribution in phase $\mathrm{I}(\mathrm{t}=1 \mathrm{~ms})$ for the full and interface slip models.

Fig. 11 Variation of energy terms for the reference drop impact simulation.

Fig. 12 Variation of the change of the kinetic energy for drop impact simulation with different grid resolution (a) interface slip with constant slip ratio (b) interface slip with constant slip length (c) momentum slip with constant slip ratio (d) momentum slip with constant slip length.

Fig. 13 Variation of the change of the surface energy for drop impact simulation with different grid resolution (a) interface slip with constant slip ratio (b) interface slip with constant slip length (c) momentum slip with constant slip ratio (d) momentum slip with constant slip length.

Fig. 14 Variation of the change of the viscous dissipation energy for drop impact simulation with different grid resolution (a) interface slip with constant slip ratio (b) interface slip with constant slip length (c) momentum slip with constant slip ratio (d) momentum slip with constant slip length. 
Fig. 15 Wall shear stress distribution in phase $\mathrm{I}(\mathrm{t}=1 \mathrm{~ms})$ for (a) interface slip $(\lambda / \Delta x=0.5)$ and (b) momentum slip model $(\lambda / \Delta x=0.25)$ with different grid resolution.

Fig. 16 Variation of the computed friction coefficient from energy analysis with different grid resolution (a) interface slip with constant slip ratio (b) interface slip with constant slip length (c) momentum slip with constant slip ratio (d) momentum slip with constant slip length.

Fig. 17 Variation of contact diameter for drop impact simulation with different grid resolution (a) interface slip with constant slip ratio (b) interface slip with constant slip length (c) momentum slip with constant slip ratio (d) momentum slip with constant slip length.

Fig. 18 Variation of the energy change terms with different slip ratio (a) kinetic energy (b) viscous dissipation (c) surface energy (d) computed friction coefficient.

Fig. 19 Contact angle distribution with contact line speed at different slip ratio (a) $\lambda / \Delta x=0.75$, (b) $\lambda / \Delta x=0.5$, (c) $\lambda / \Delta x=0.375$, (d) $\lambda / \Delta x=0.25$, (e) $\lambda / \Delta x=0.125$, (e) $\lambda / \Delta x=0.0625$.

Fig. 20 Effect of advancing contact angle on contact diameter.

Fig. 21 Variation of the energy change terms with different advancing angles (a) kinetic energy (b) viscous dissipation (c) surface energy (d) computed friction coefficient.

Fig. 22 Effect of receding contact angle on contact diameter.

Fig. 23 Variation of the energy change terms with different receding angle (a) kinetic energy (b) viscous dissipation (c) surface energy (d) computed friction coefficient.

Fig. 24 Contact diameter variation using different slip ratios for the spreading $(\lambda / \Delta x=1.0)$ and contracting $(\lambda / \Delta x=0.25)$ phases.

Fig. 25 Simulation geometry for 3D drop impact on a solid cylinder.

Fig. 26 Interface evolution for the regular surface $\left(\theta_{a d v}=114_{\mathrm{o}}, \theta_{\text {rec }}=65_{\mathrm{o}}\right)$.

Fig. 27 Interface evolution for the hydrophilic surface $\left(\theta_{a d v}=165_{\circ}, \theta_{r e c}=160_{\circ}\right)$.

Fig. 28 Interface evolution for the super-hydrophobic surface $\left(\theta_{a d v}=60_{\mathrm{o}}, \theta_{\text {rec }}=34 \mathrm{o}\right)$. 


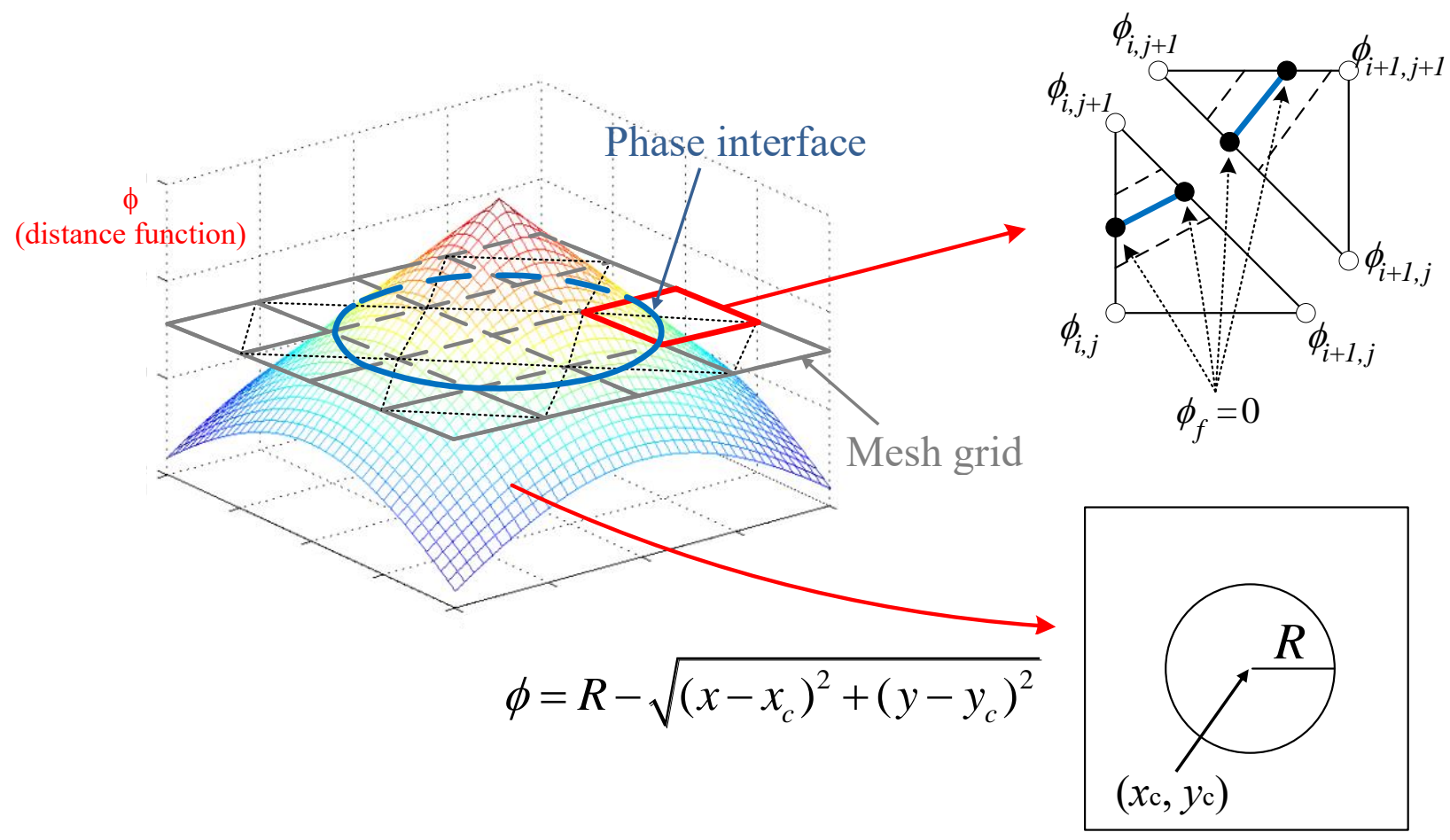

Fig. 1 


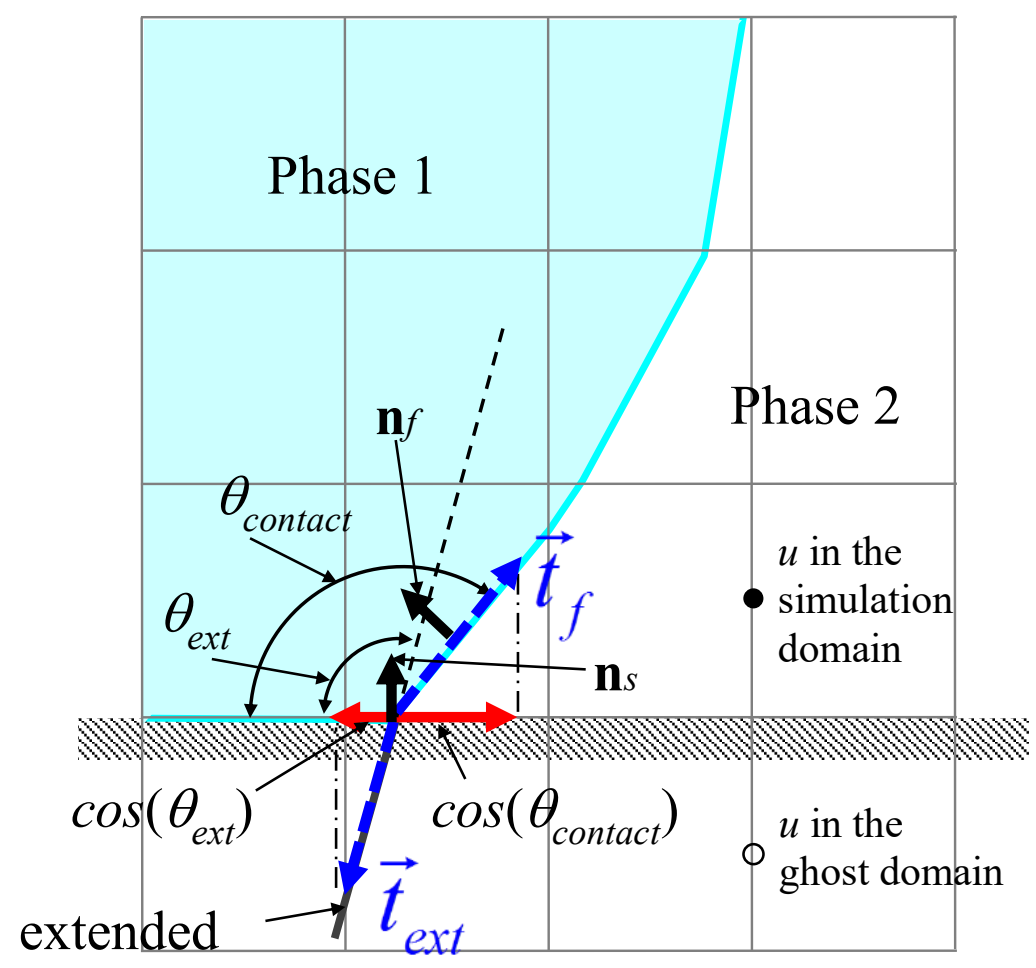

surface

(a)

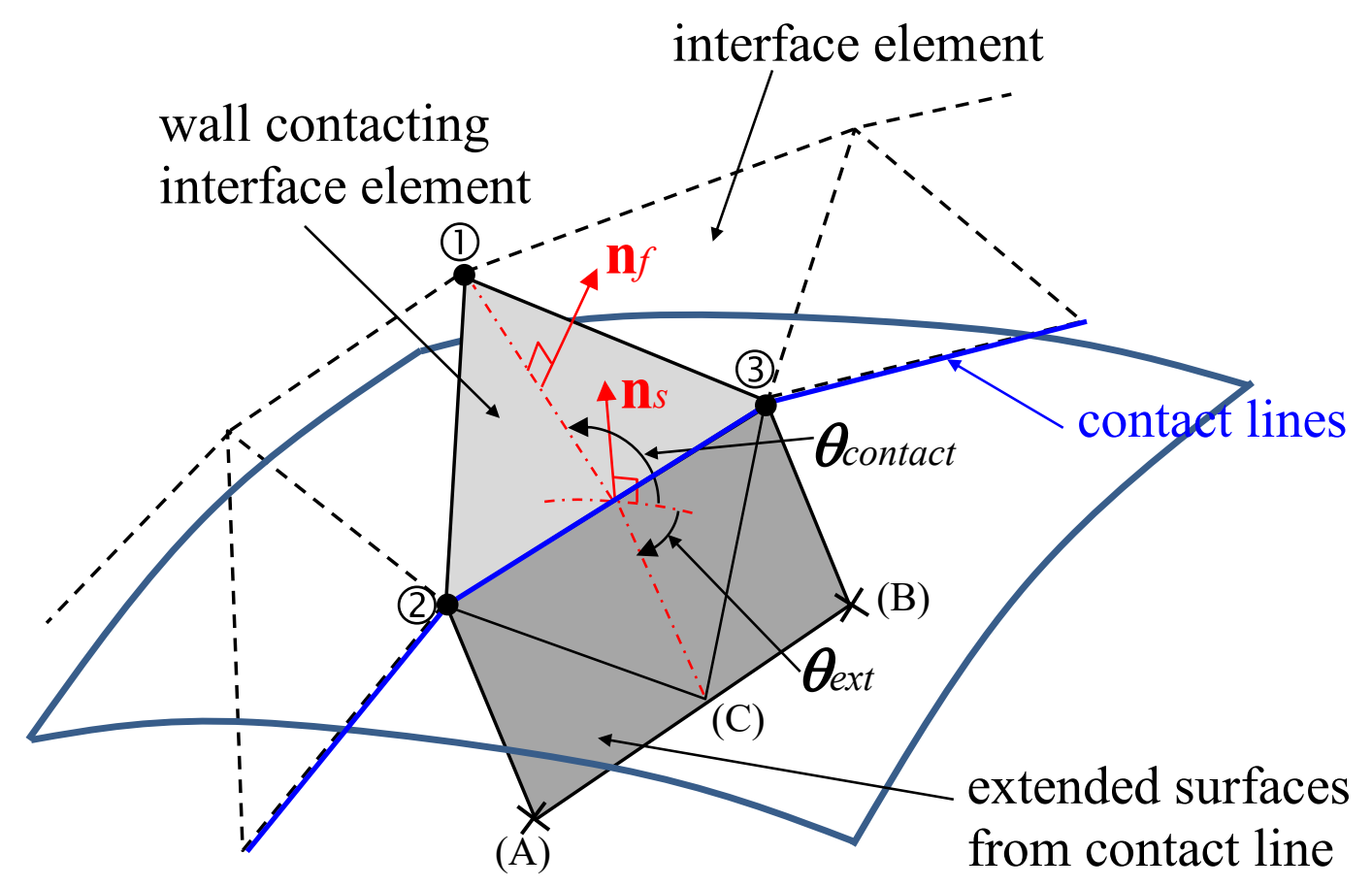

(b)

Fig. 2 

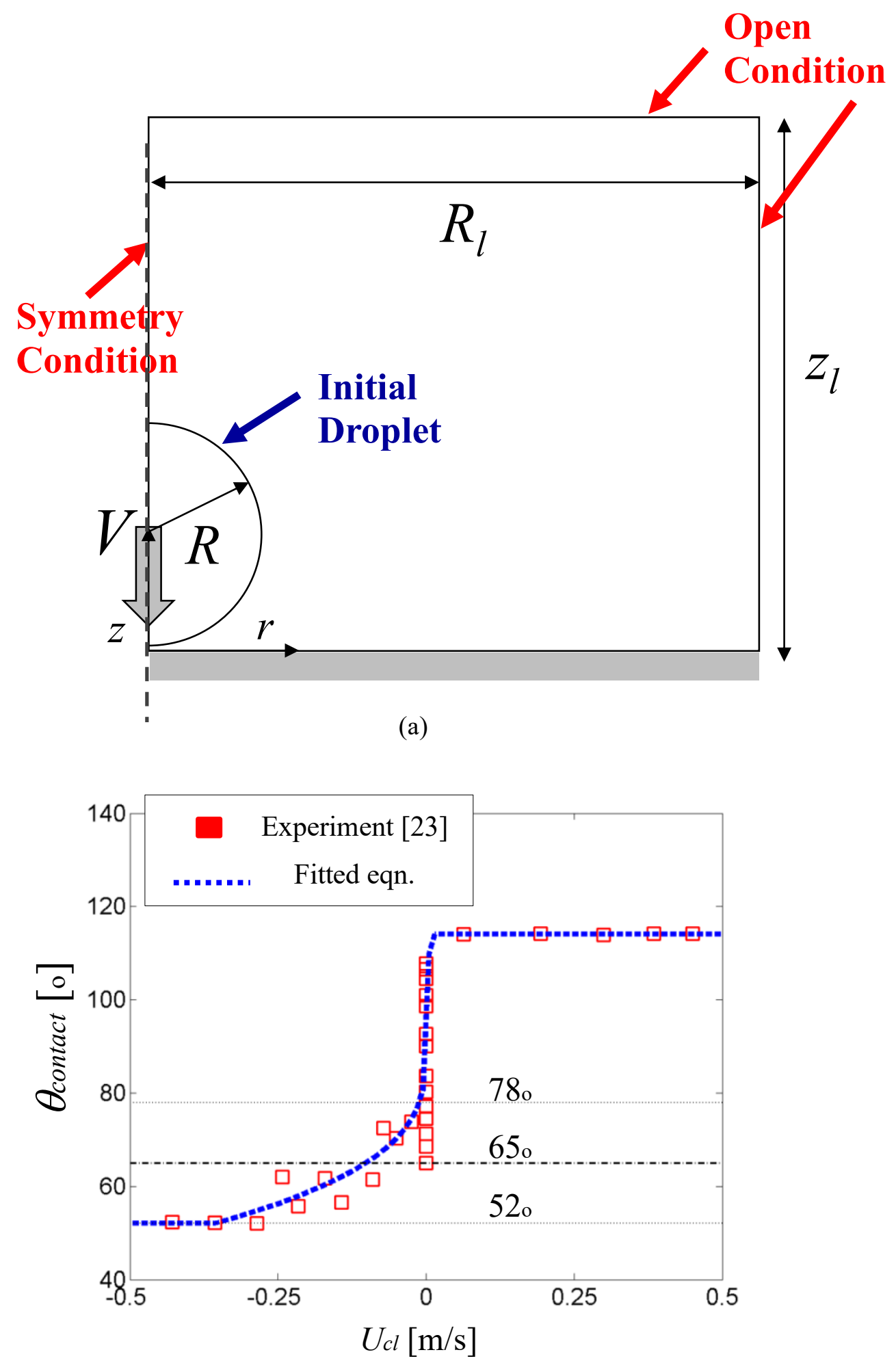

(b)

Fig. 3 


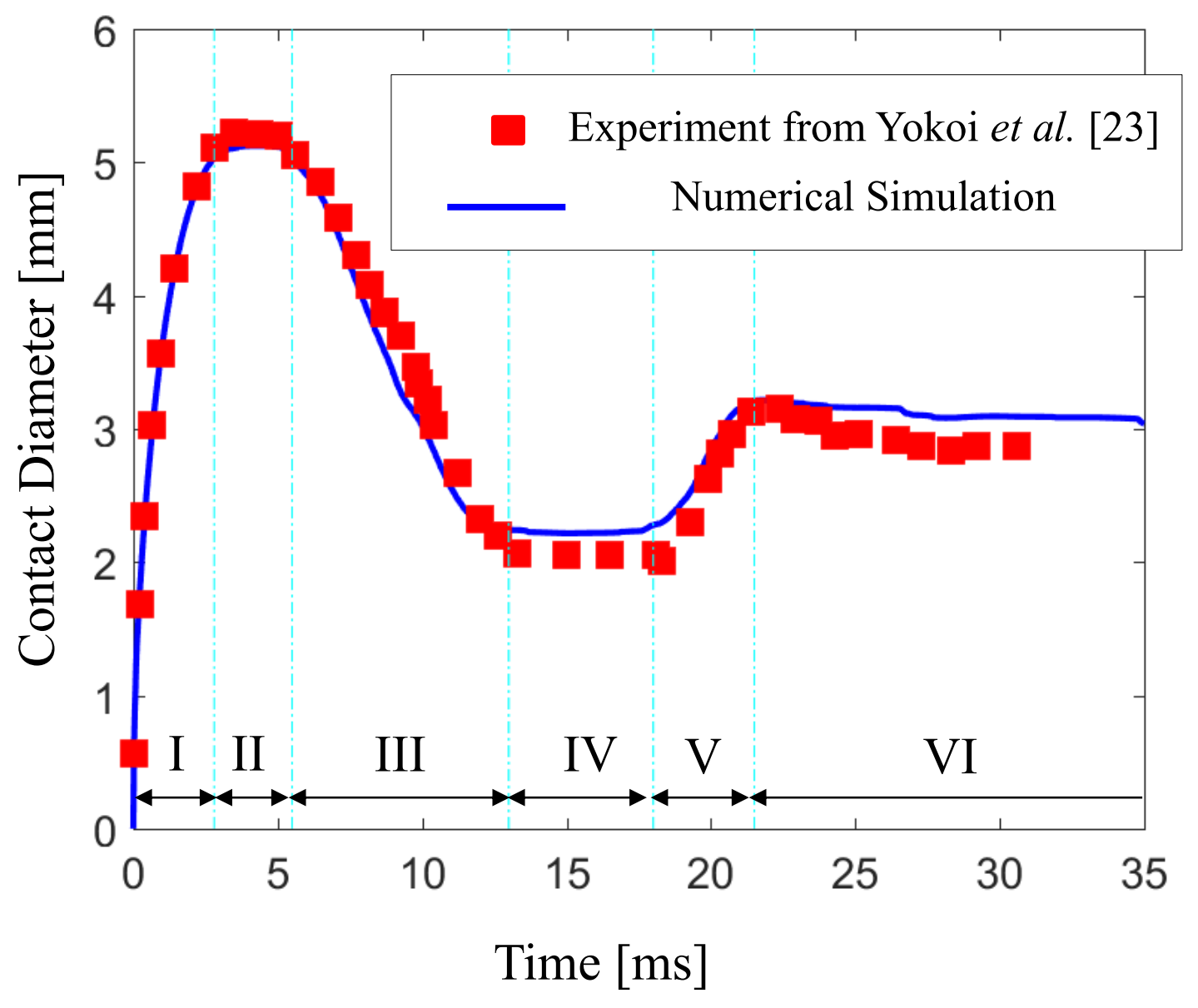

Fig. 4 


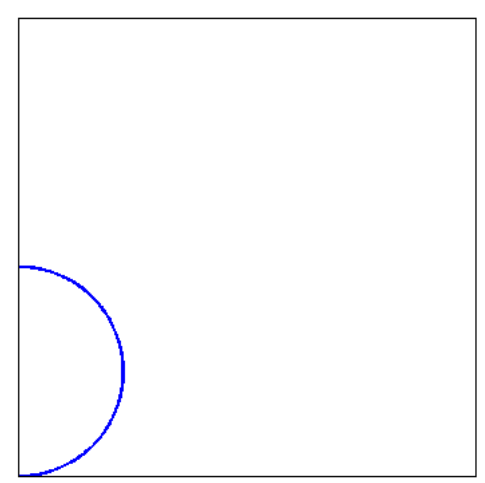

(a) $\mathrm{t}=0$

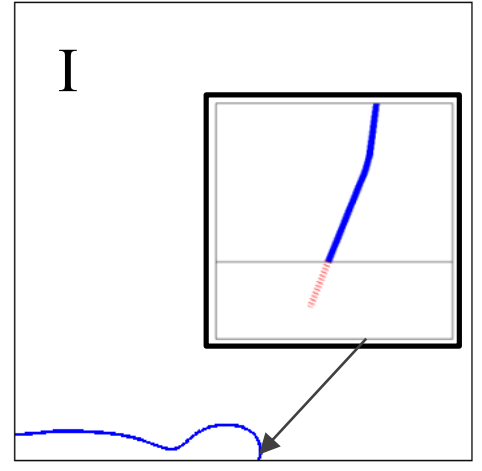

(c) $\mathrm{t}=3[\mathrm{~ms}]$

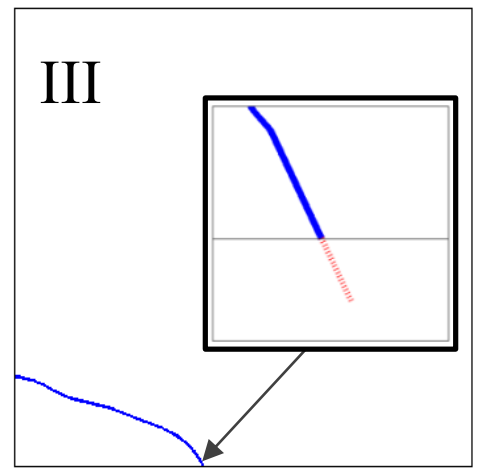

(e) $\mathrm{t}=8[\mathrm{~ms}]$

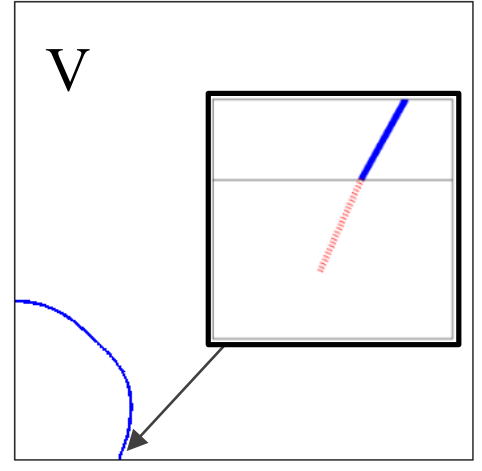

(g) $\mathrm{t}=20$ [ms]

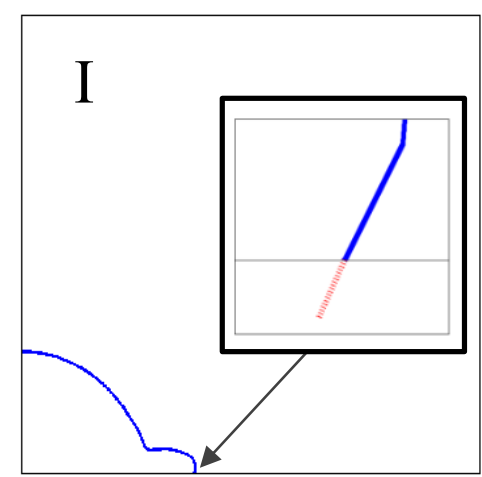

(b) $\mathrm{t}=1[\mathrm{~ms}]$

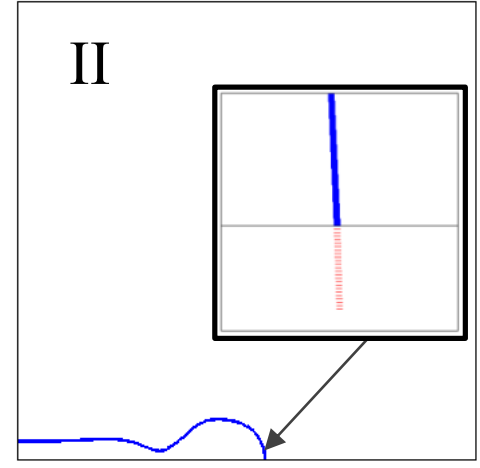

(d) $\mathrm{t}=4[\mathrm{~ms}]$

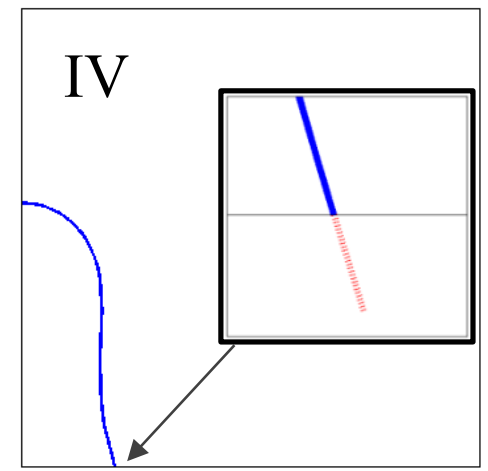

(f) $\mathrm{t}=15$ [ms]

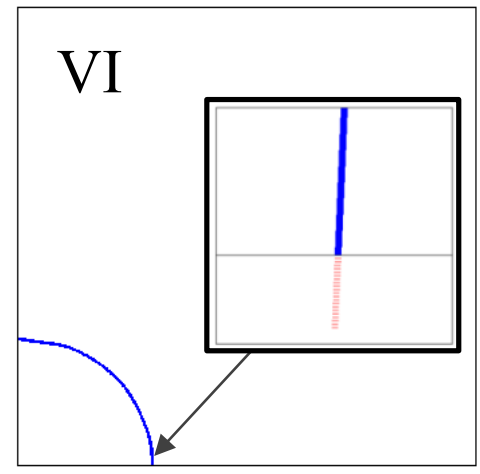

(h) $\mathrm{t}=30[\mathrm{~ms}]$

Fig. 5 

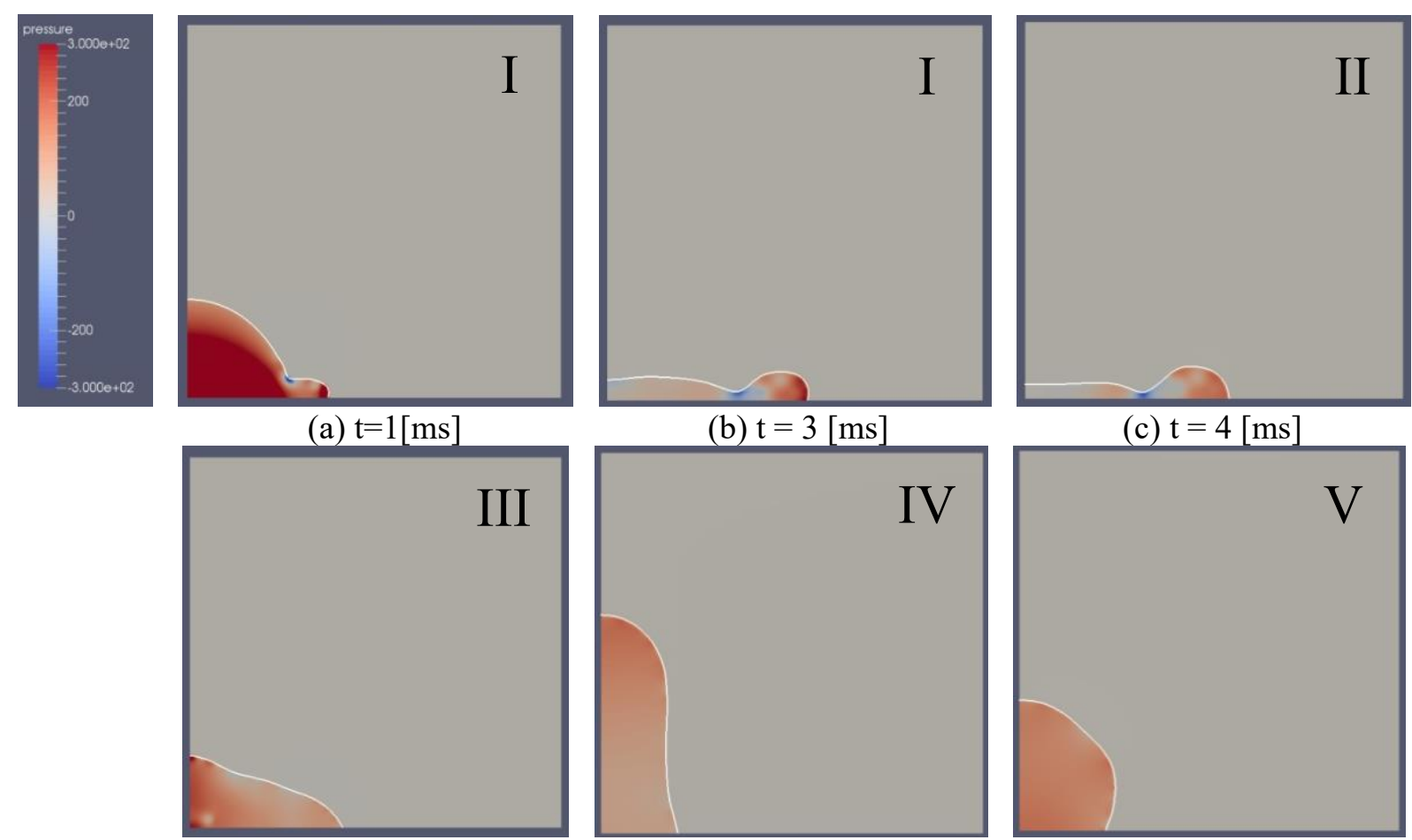

(d) $\mathrm{t}=8$ [ms]

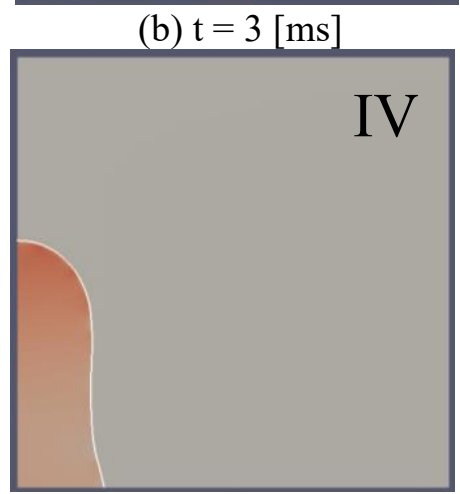

(e) $\mathrm{t}=15[\mathrm{~ms}]$

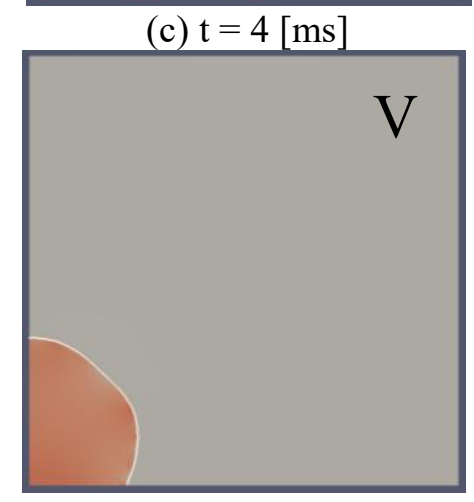

(f) $\mathrm{t}=20[\mathrm{~ms}]$

Fig. 6 


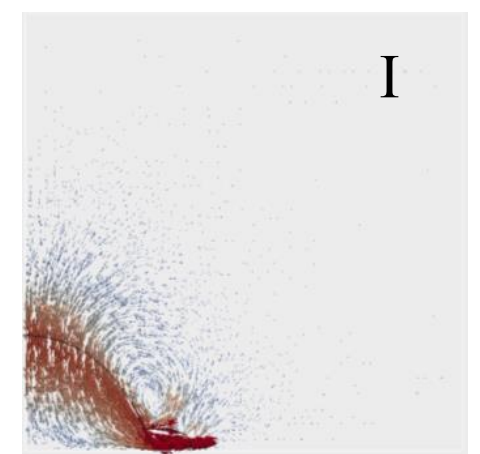

(a) $\mathrm{t}=1[\mathrm{~ms}]$

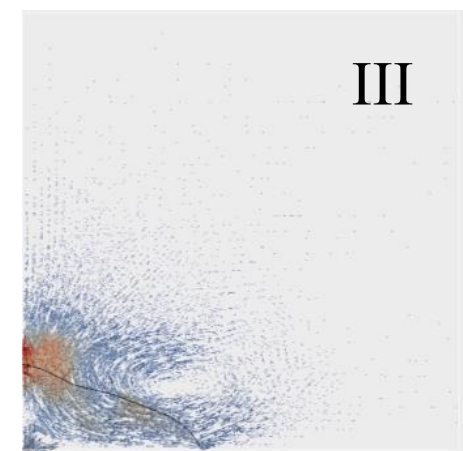

(d) $\mathrm{t}=8[\mathrm{~ms}]$

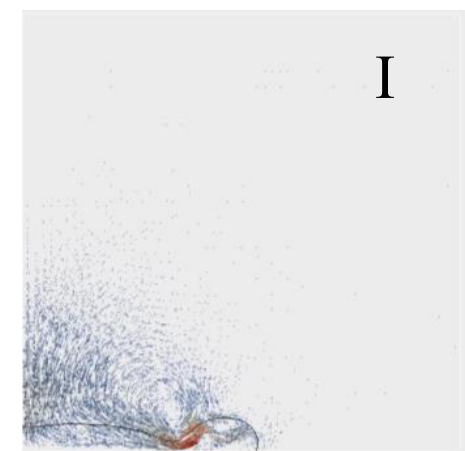

(b) $\mathrm{t}=3[\mathrm{~ms}]$

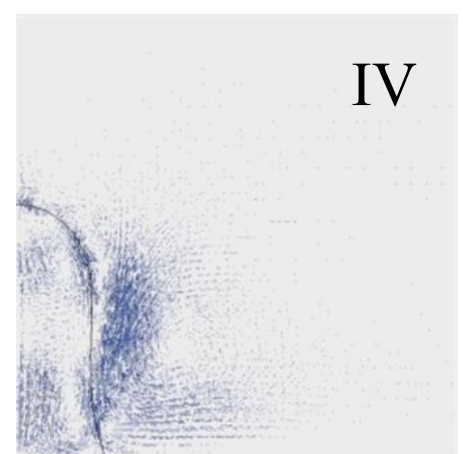

(e) $\mathrm{t}=15$ [ms]

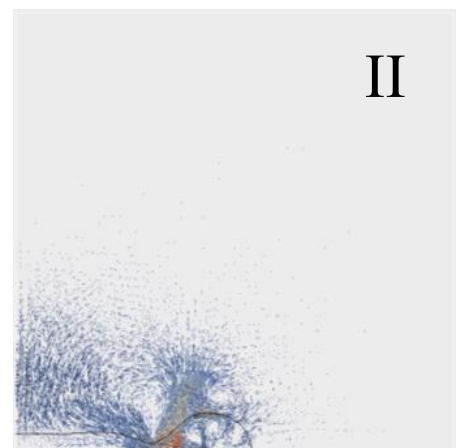

(c) $\mathrm{t}=4[\mathrm{~ms}]$

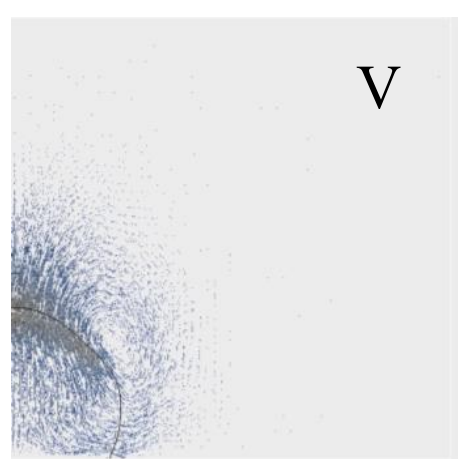

(f) $\mathrm{t}=20[\mathrm{~ms}]$

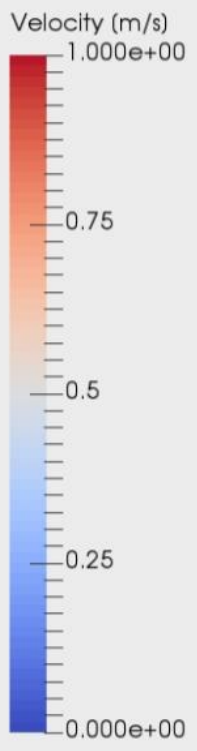

Fig. 7 


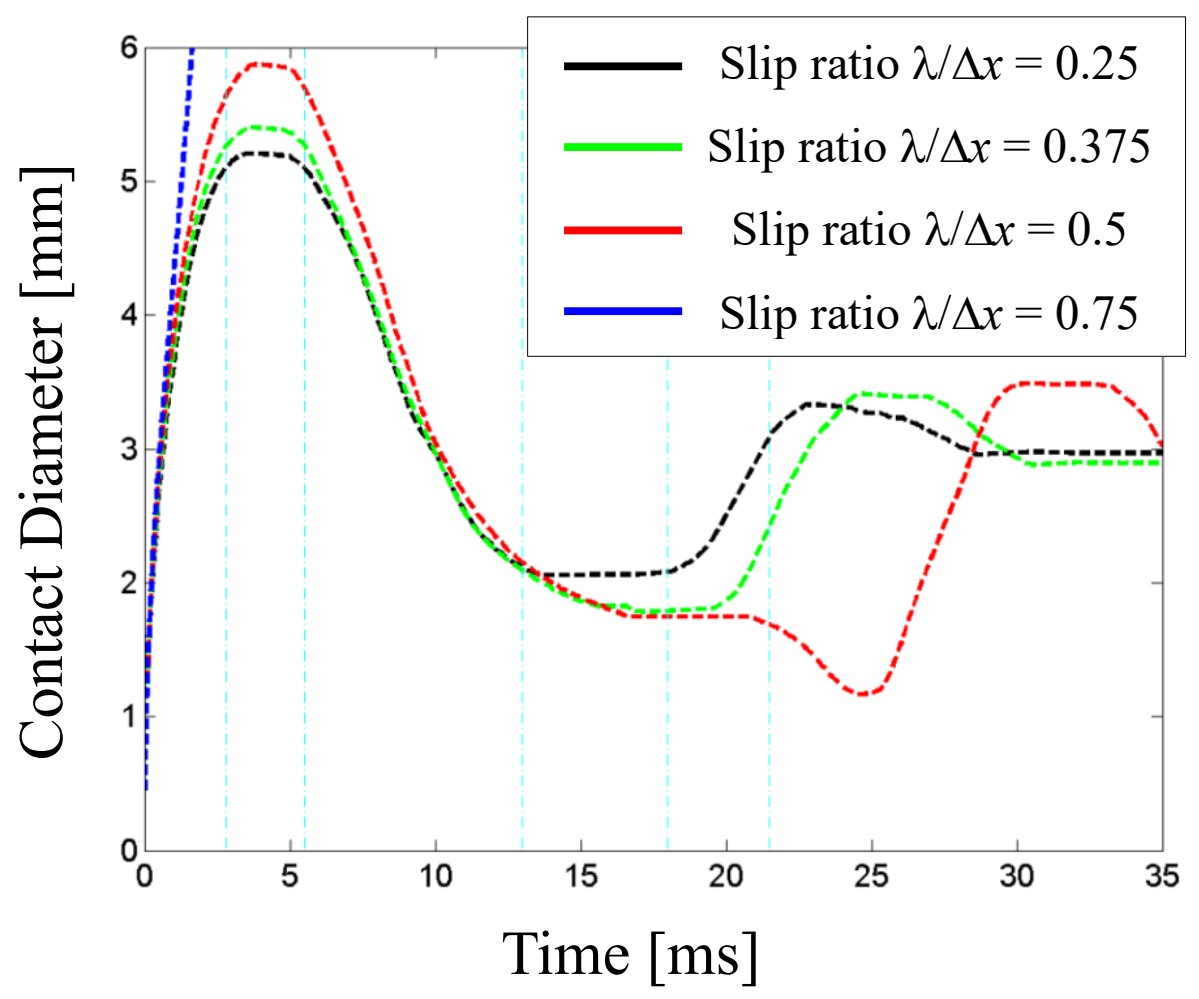

(a)

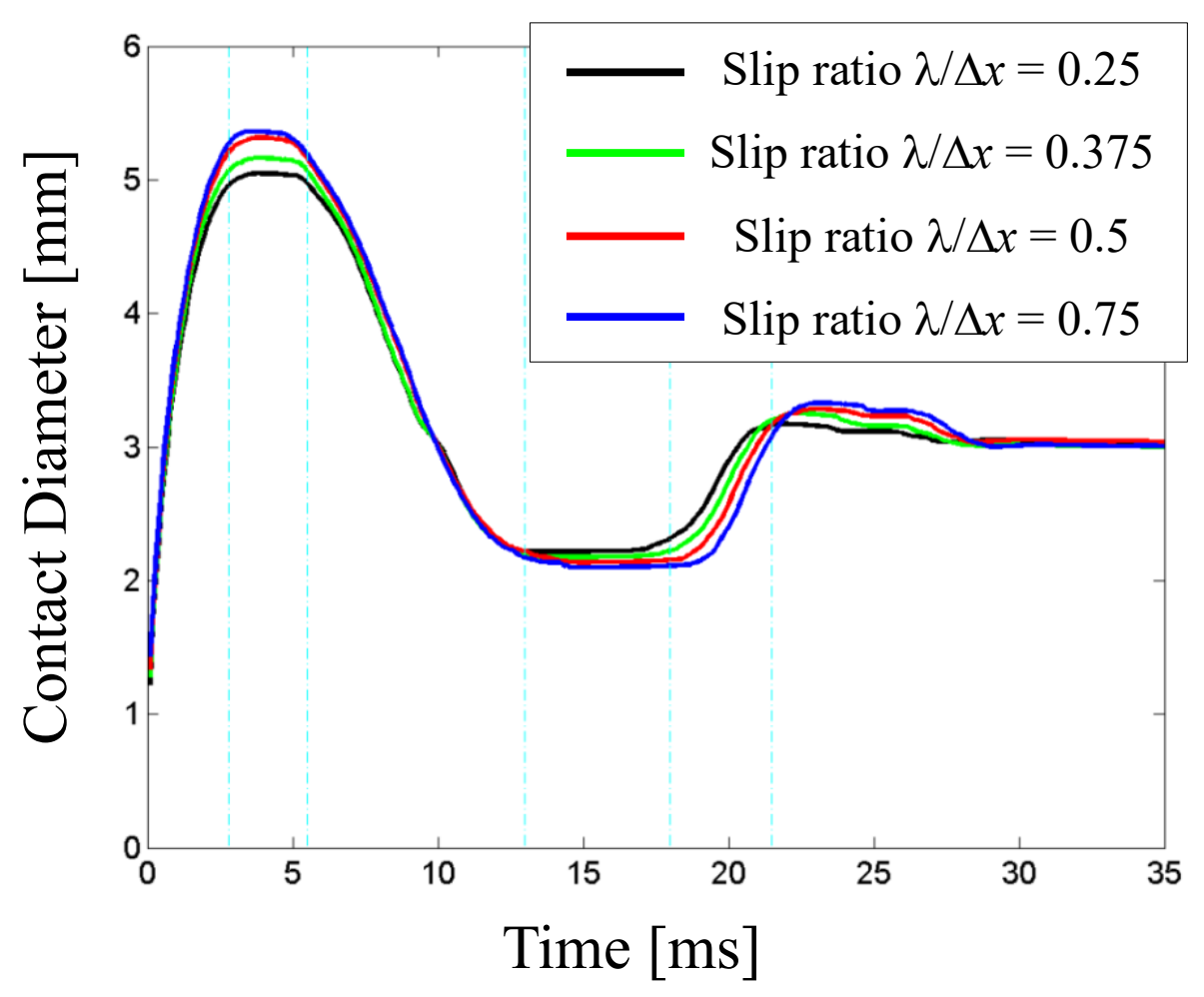

(b)

Fig. 8 


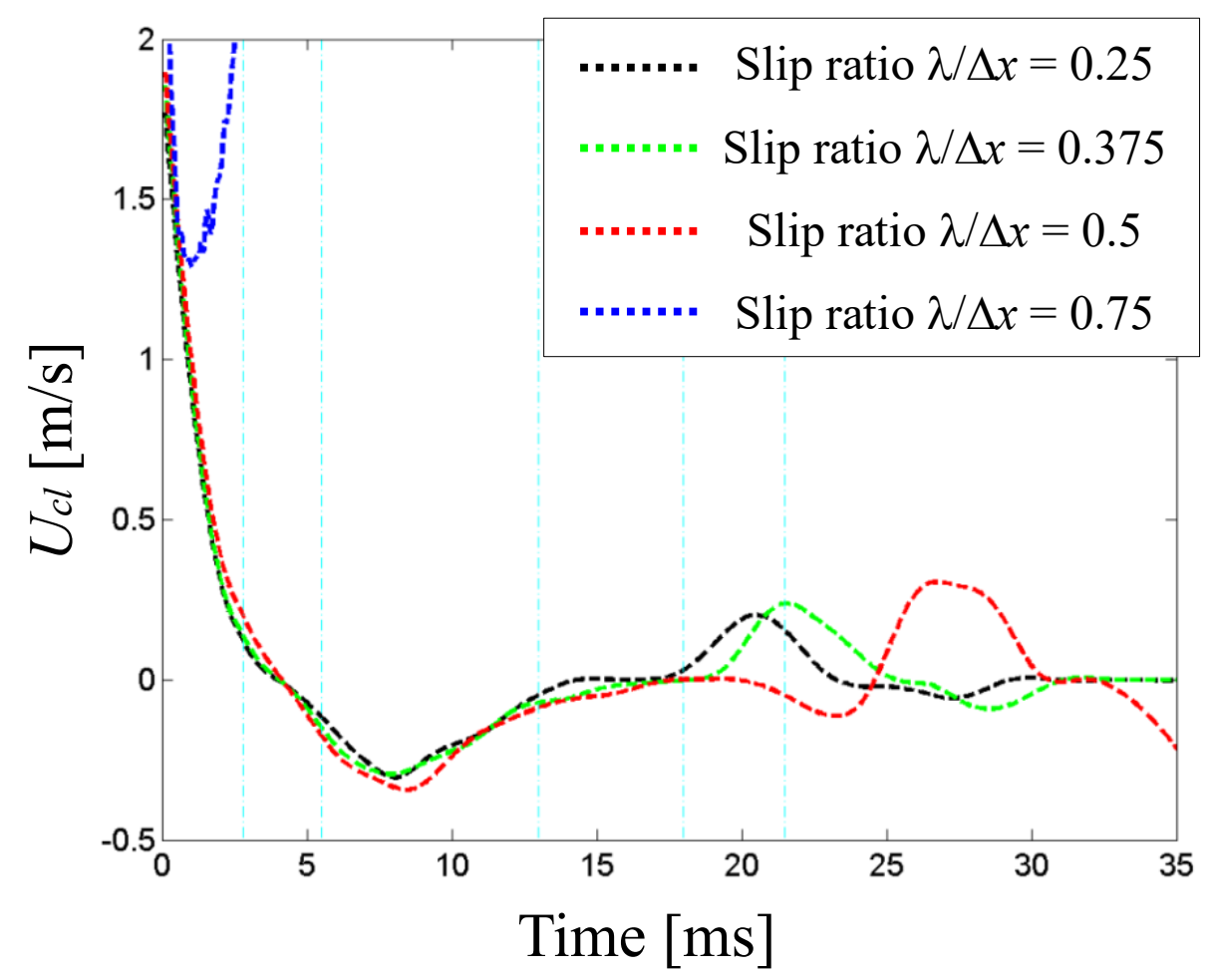

(a)

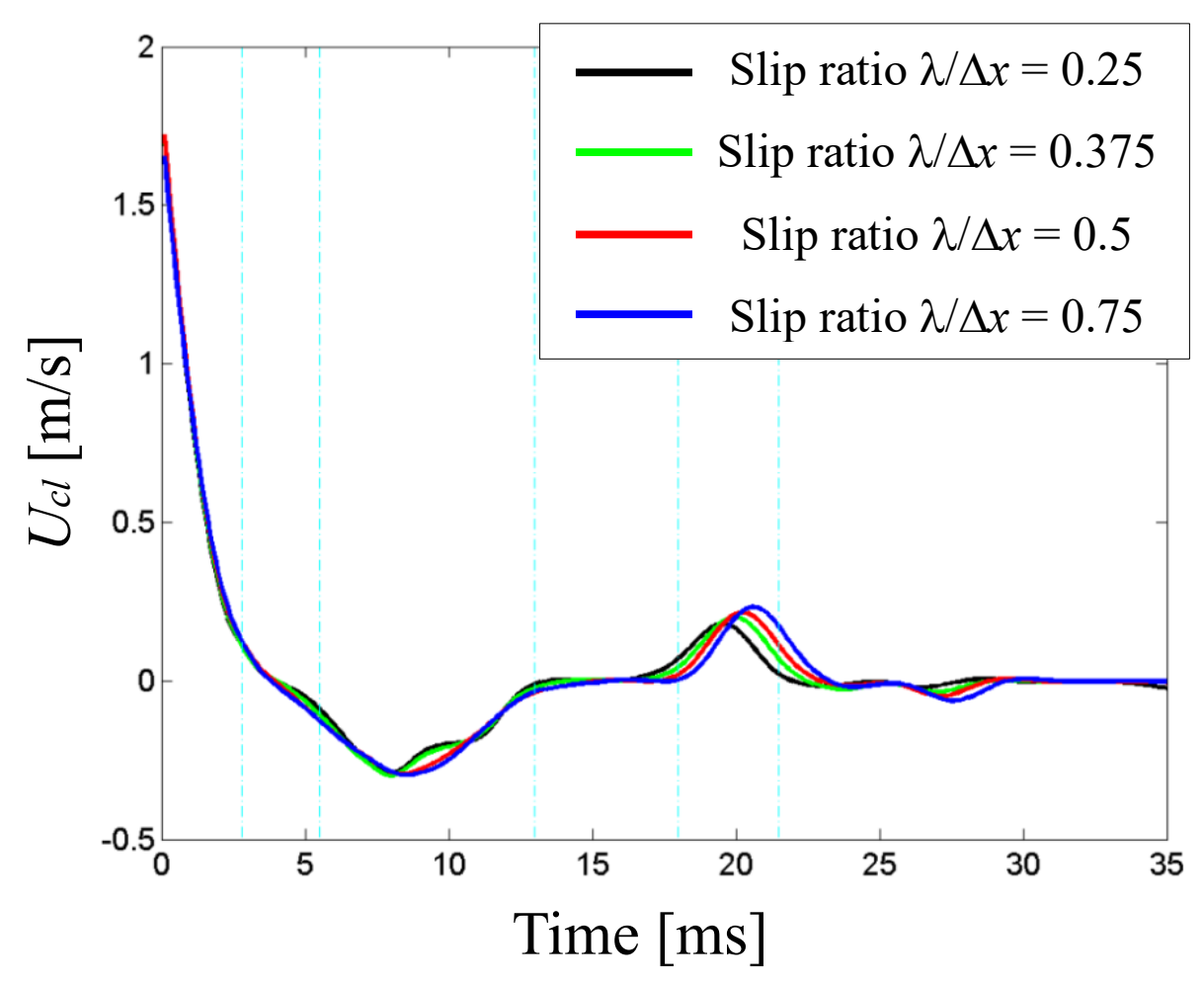

(b)

Fig. 9 


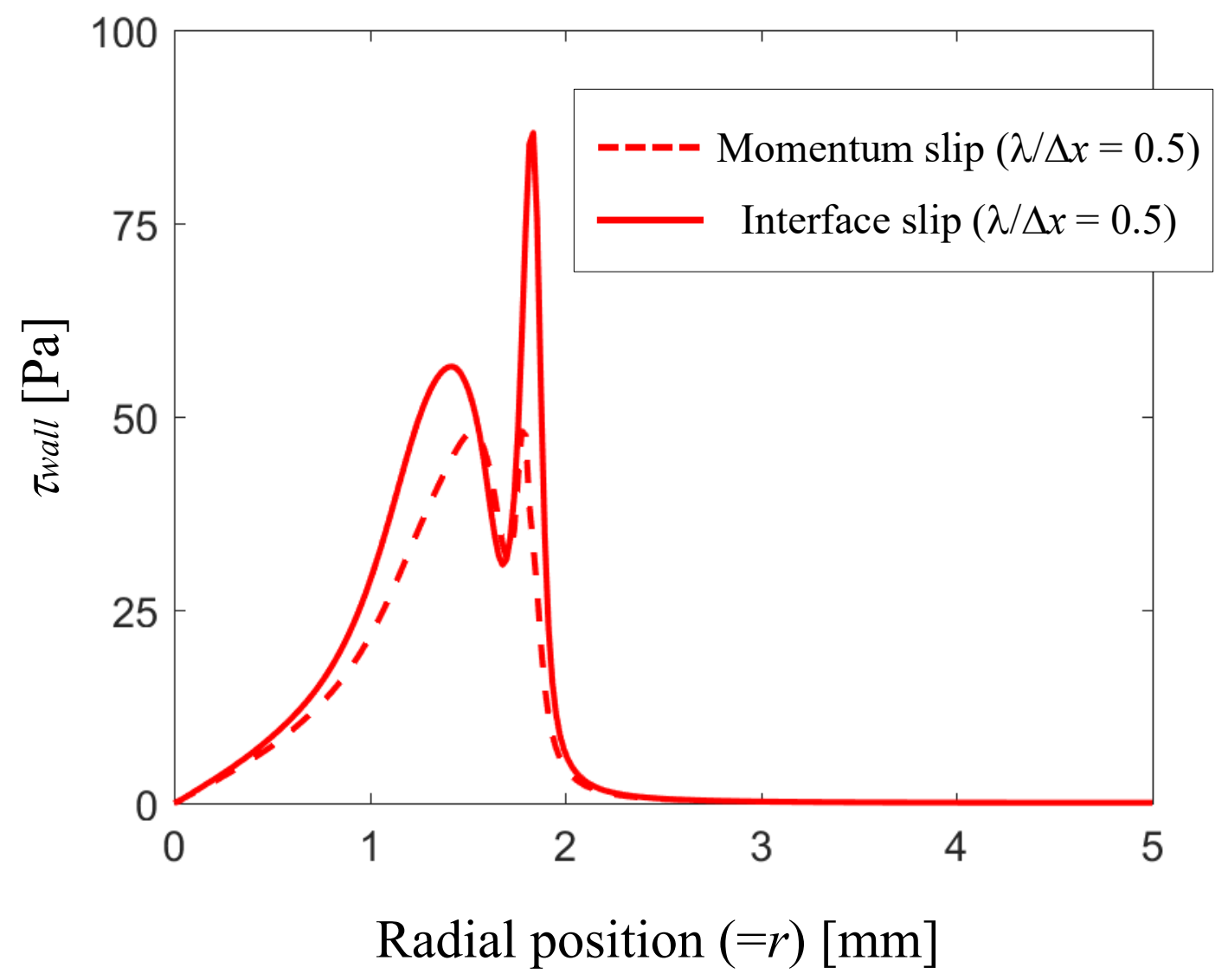

Fig. 10 


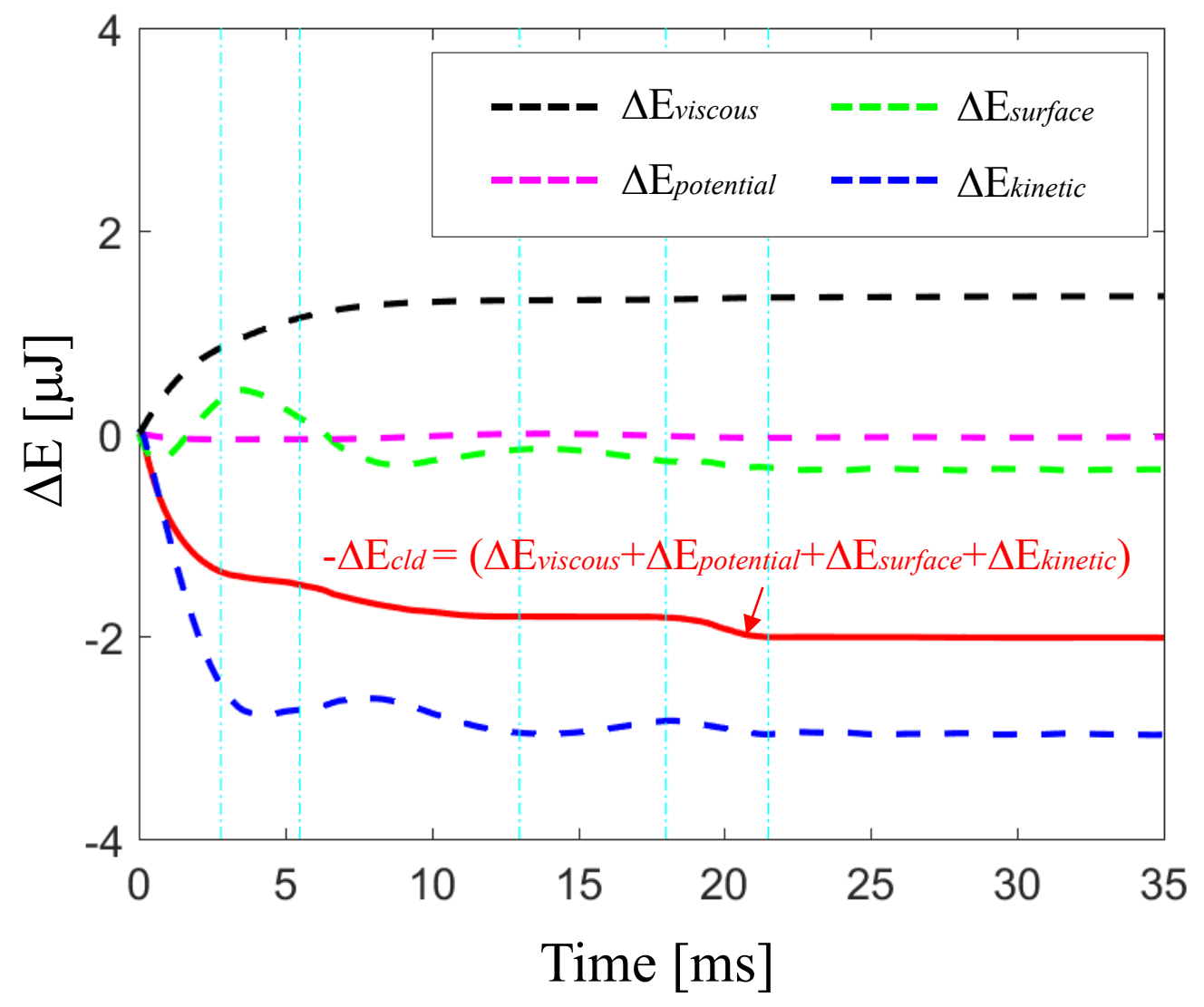

Fig. 11 


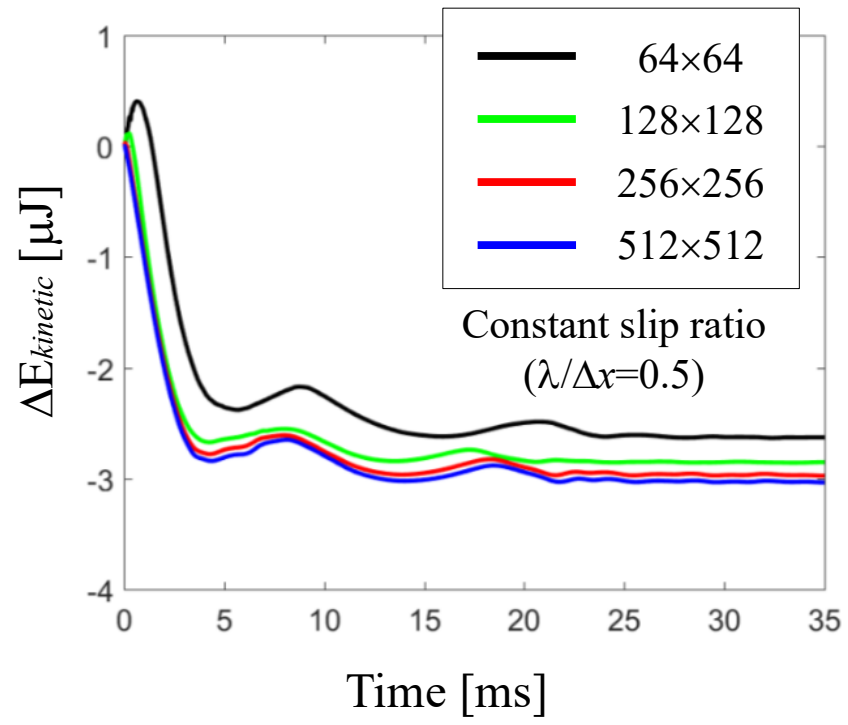

(a)

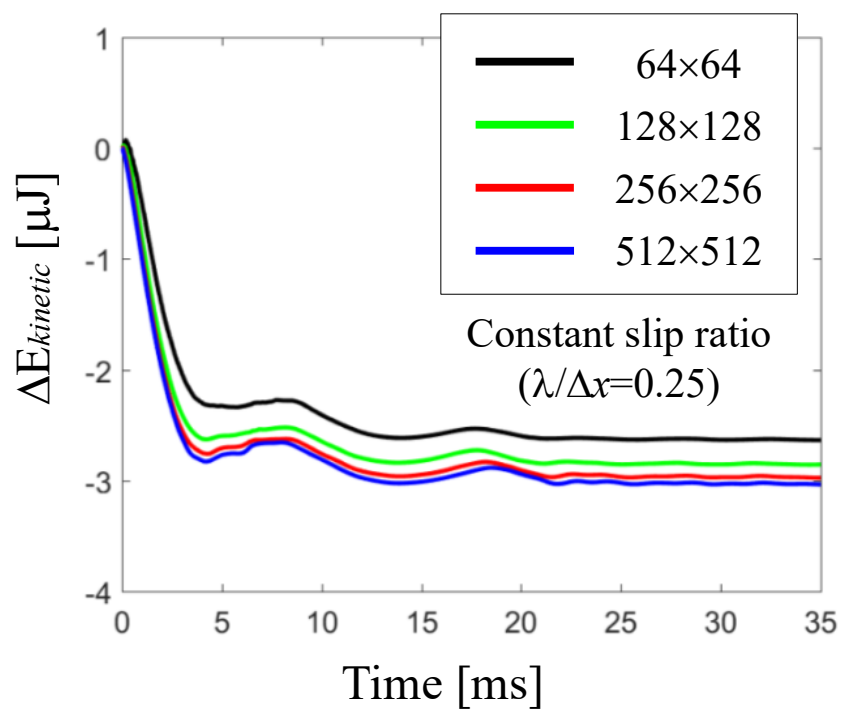

(c)

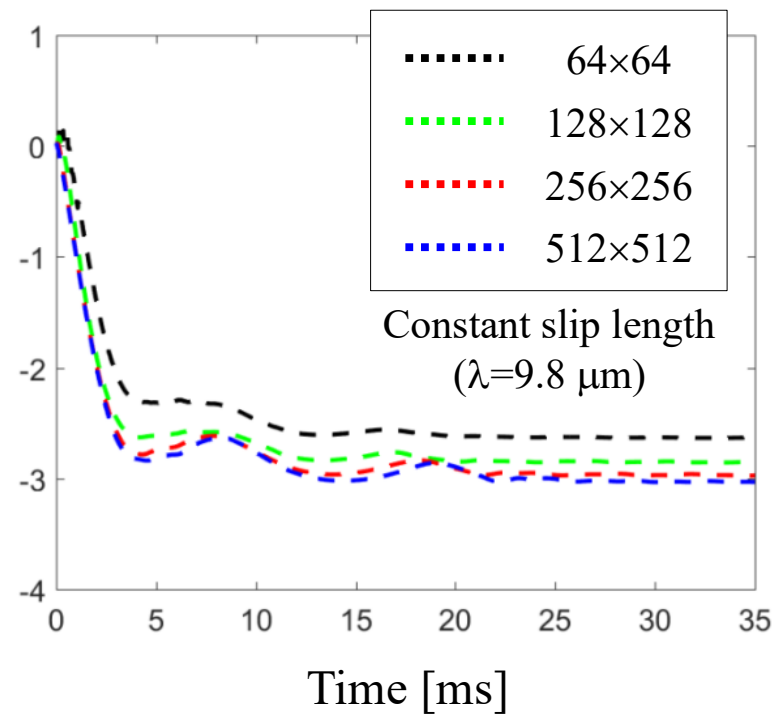

(b)

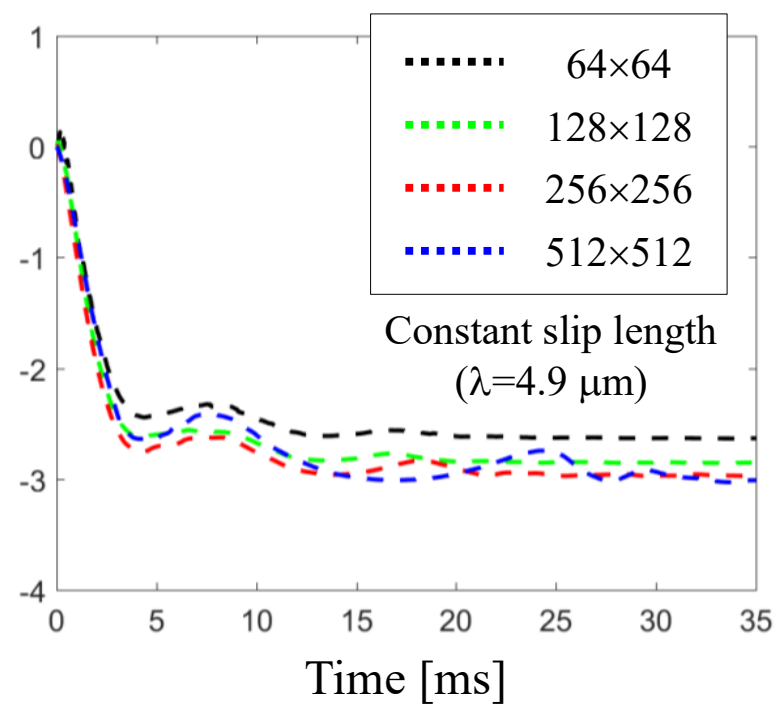

(d)

Fig. 12 


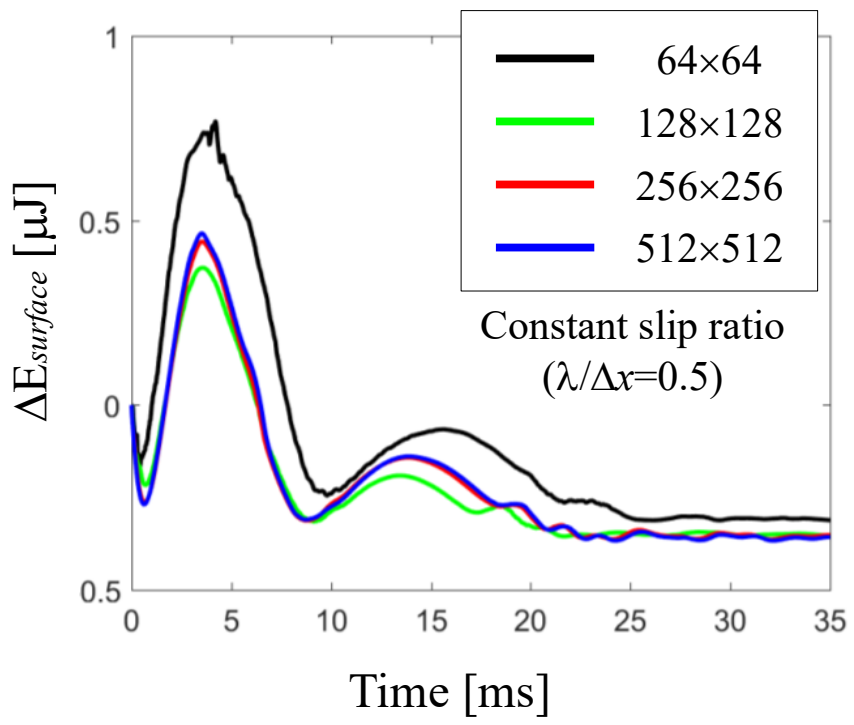

(a)

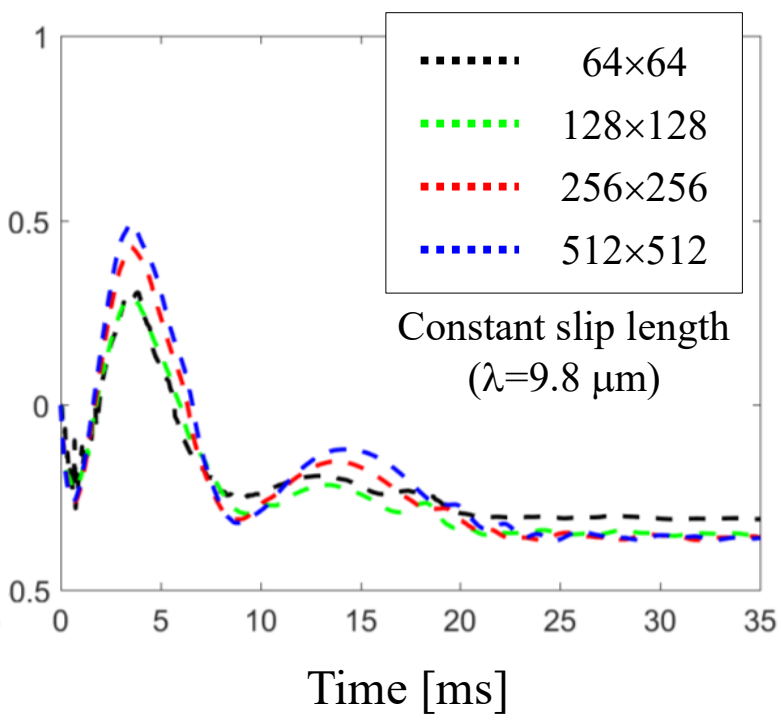

(b)

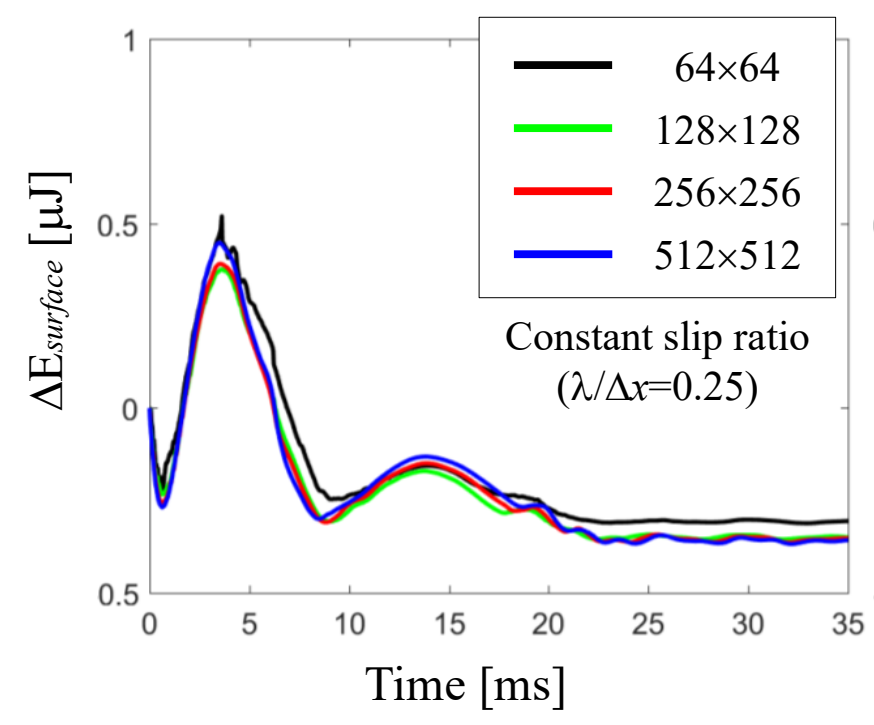

(c)

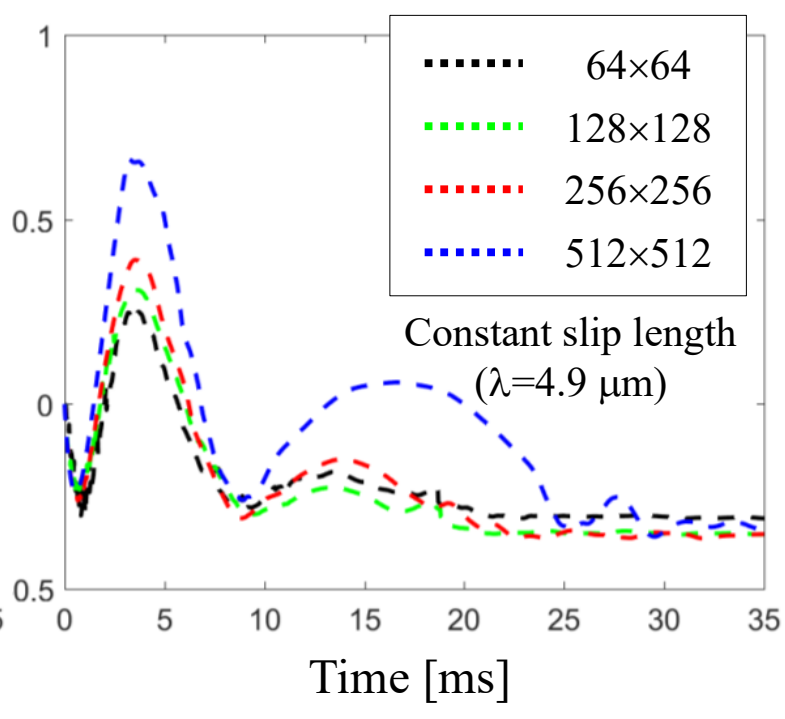

(d)

Fig. 13 


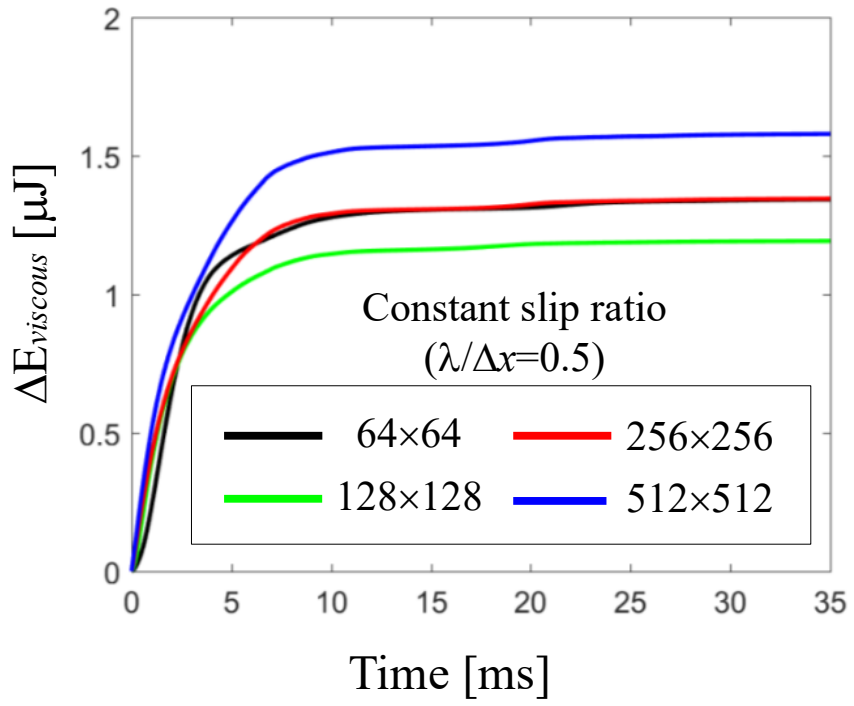

(a)

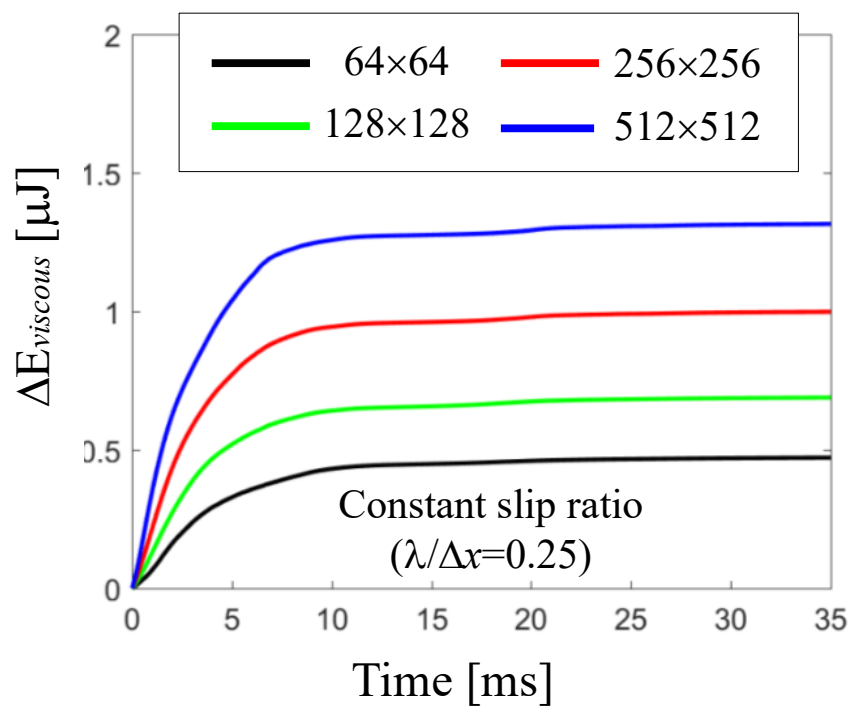

(c)

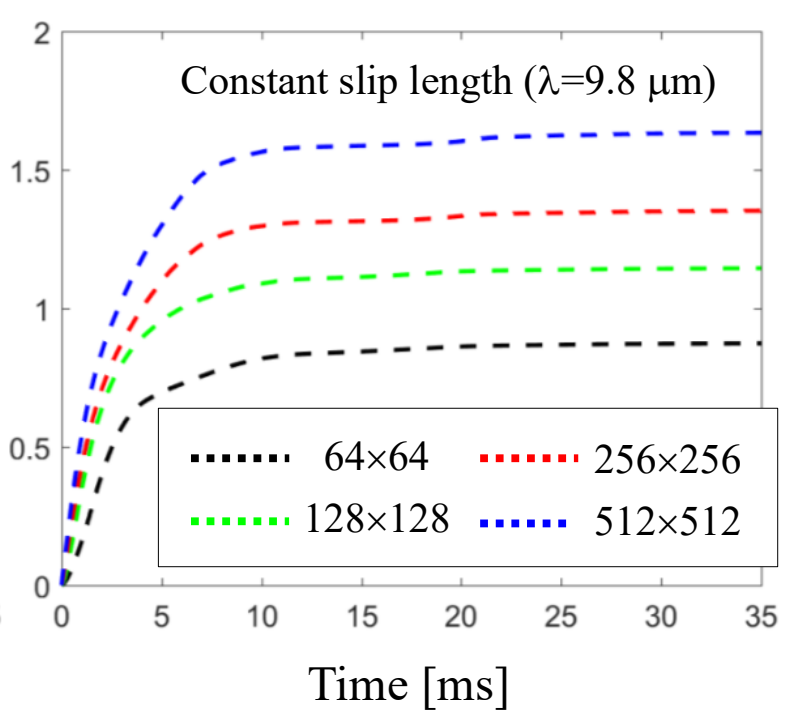

(b)

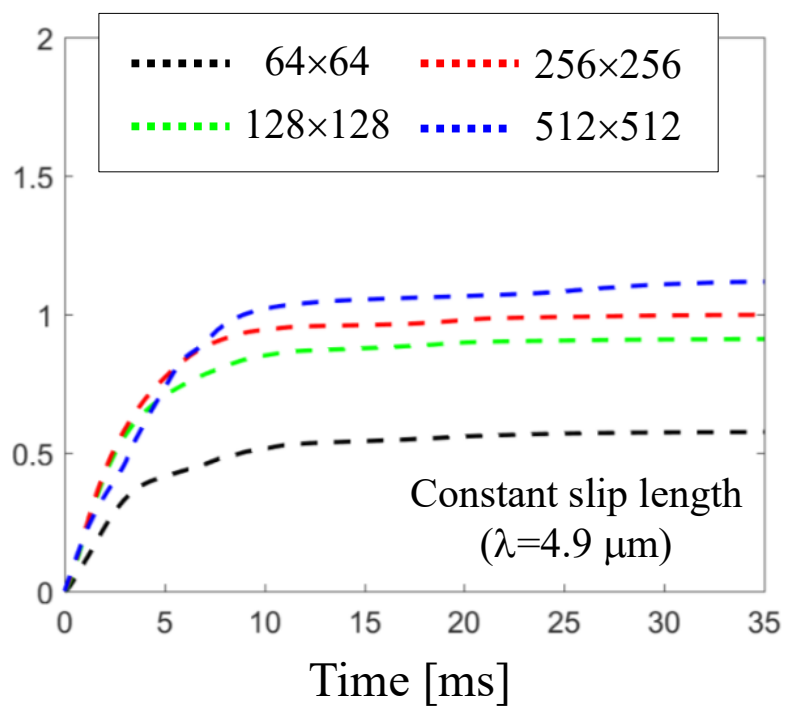

(d)

Fig. 14 


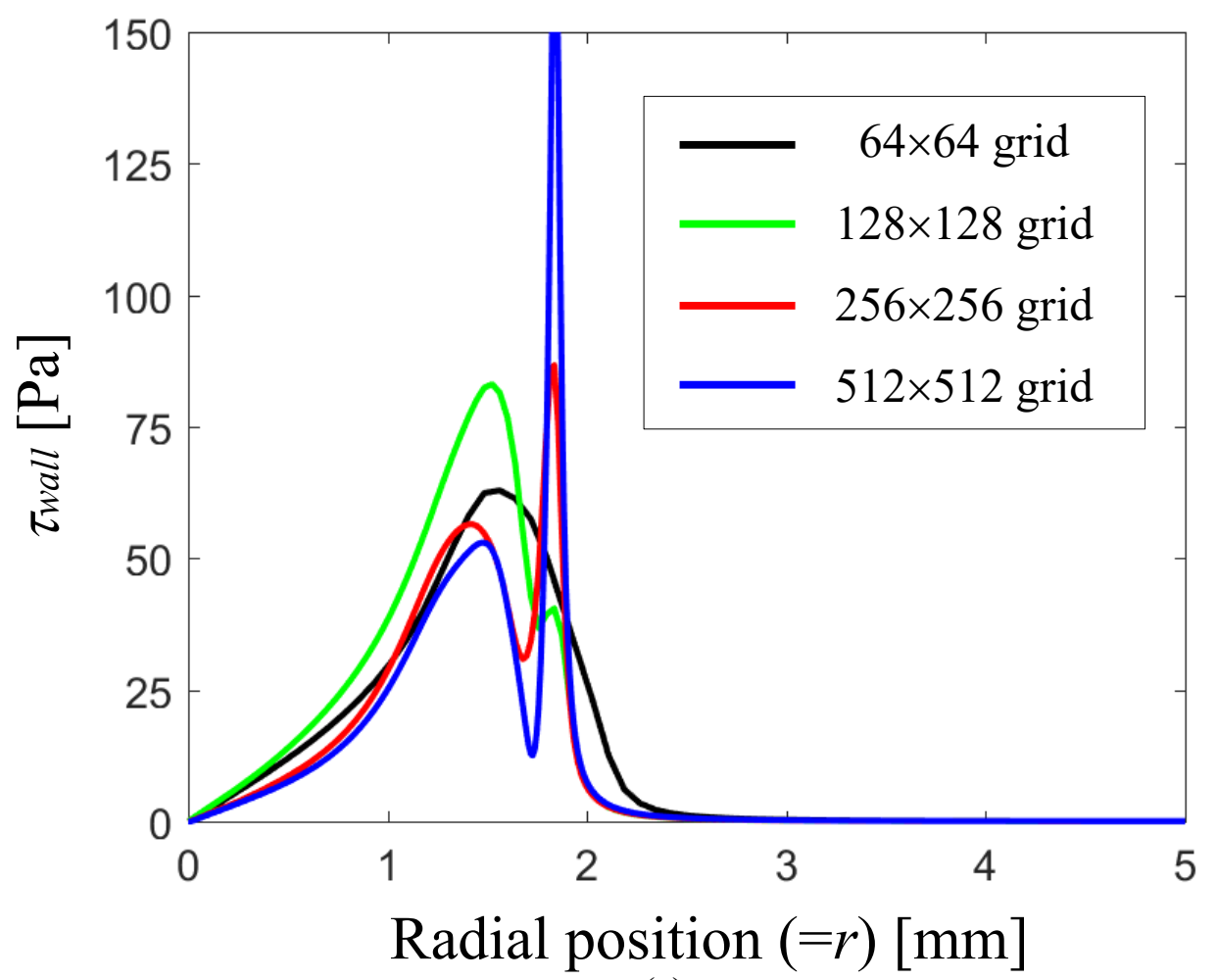

(a)

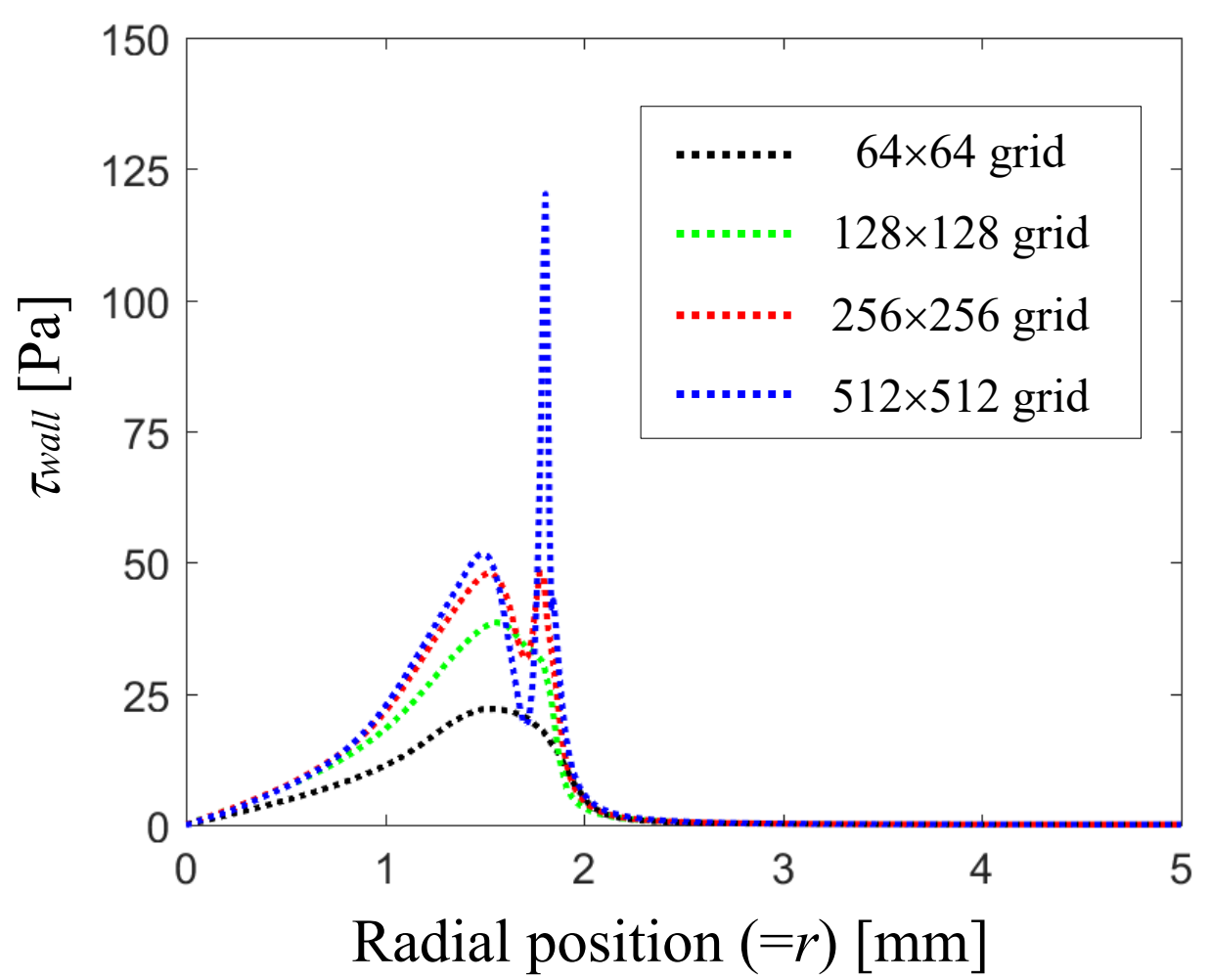

(b)

Fig. 15 


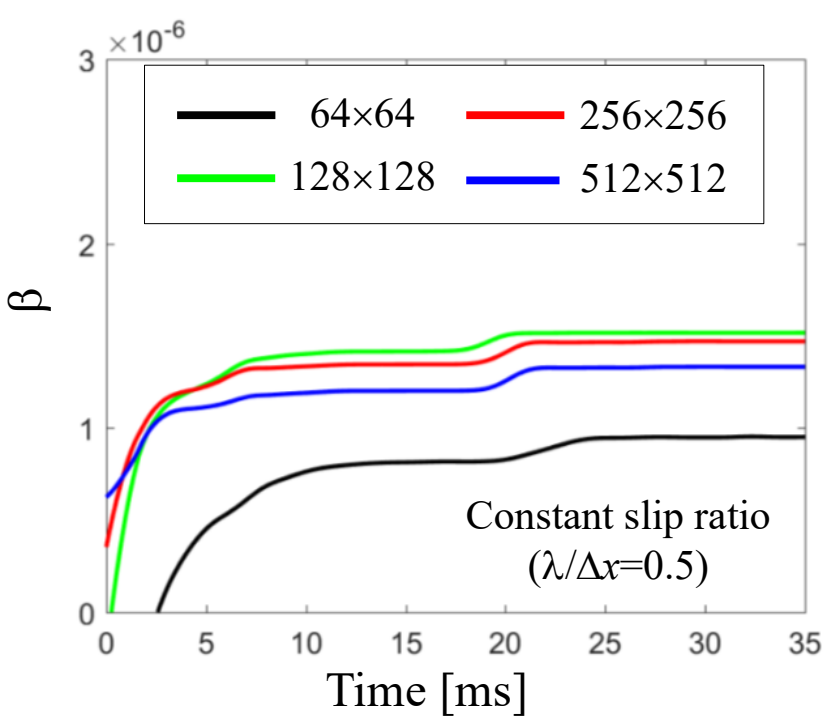

(a)

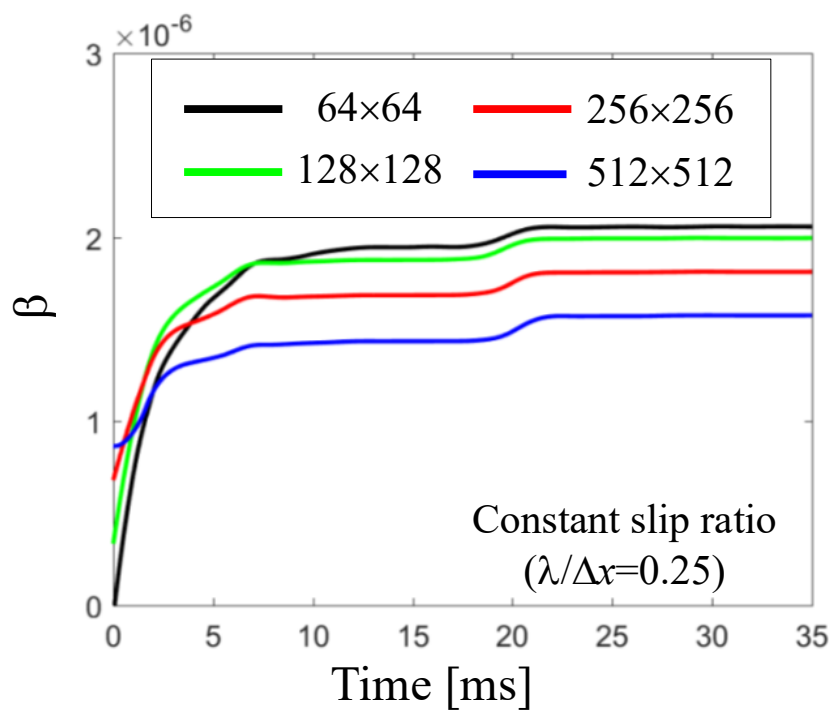

(c)

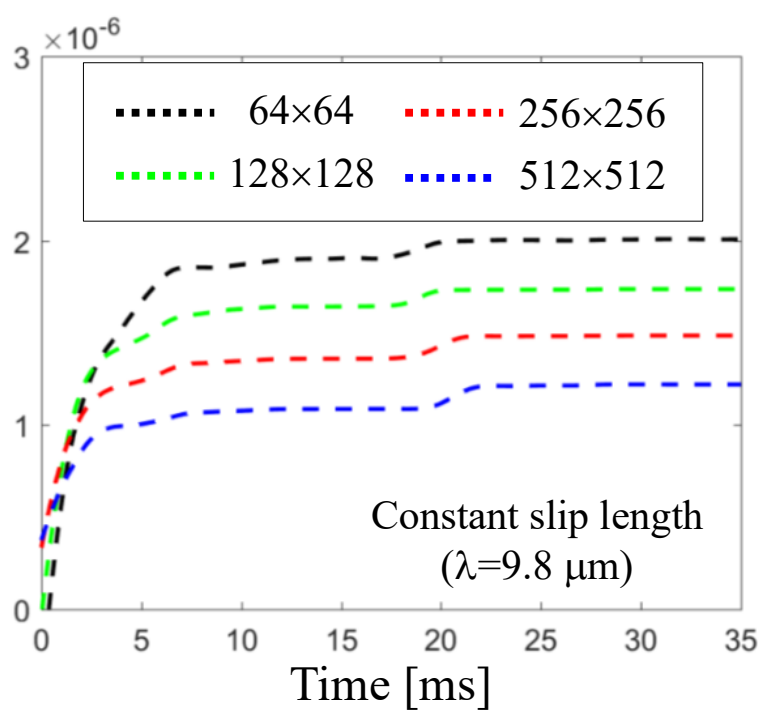

(b)

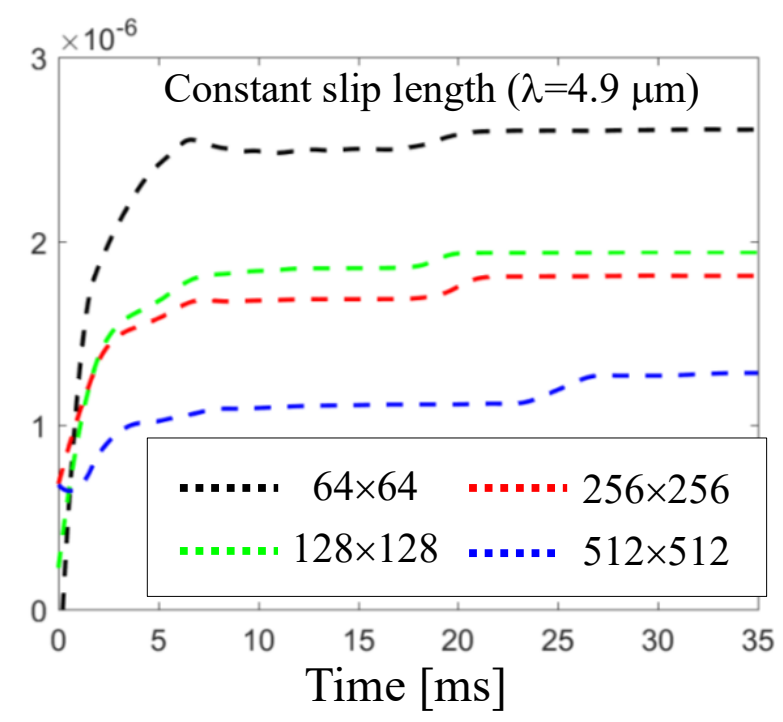

(d)

Fig. 16 


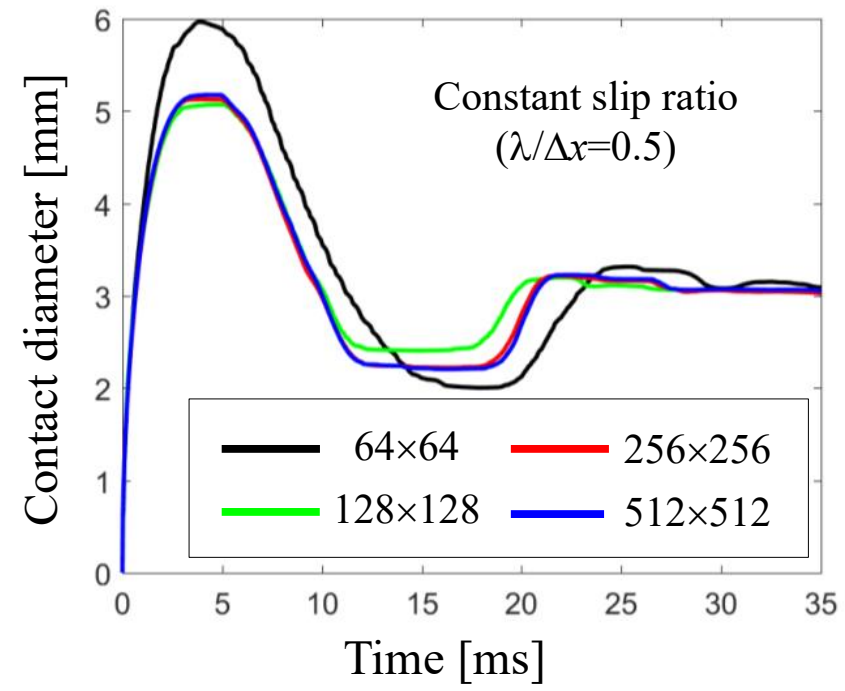

(a)

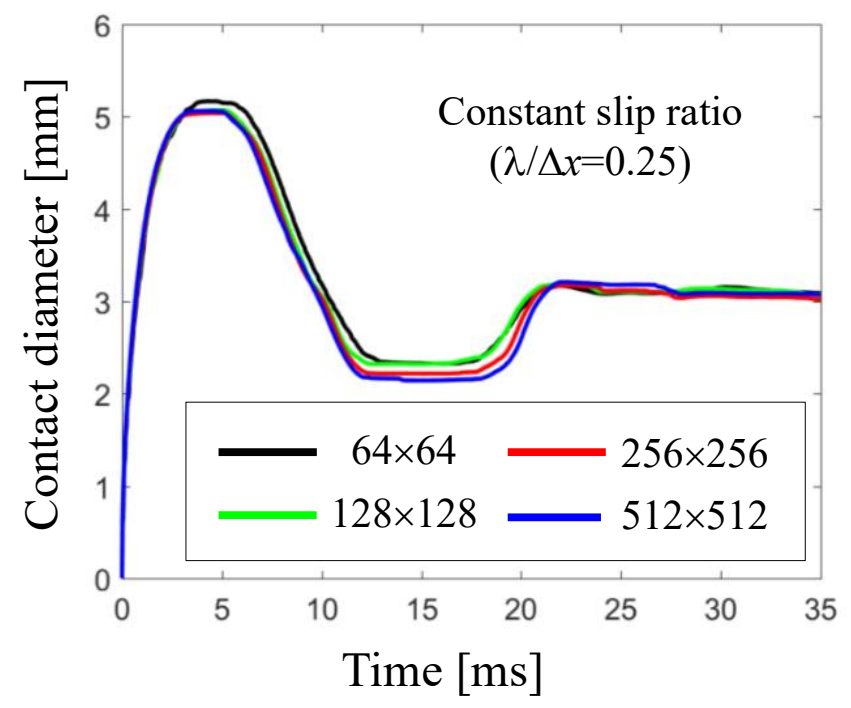

(c)

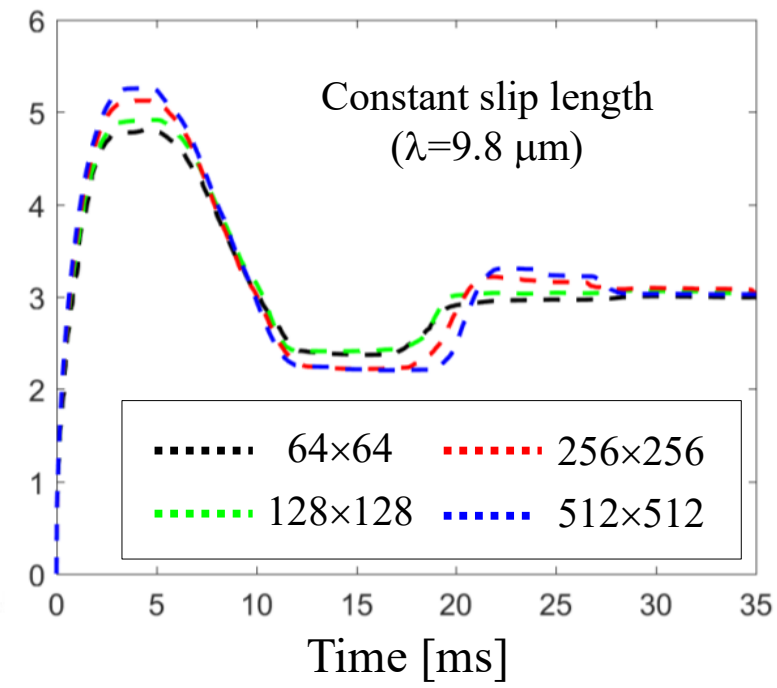

(b)

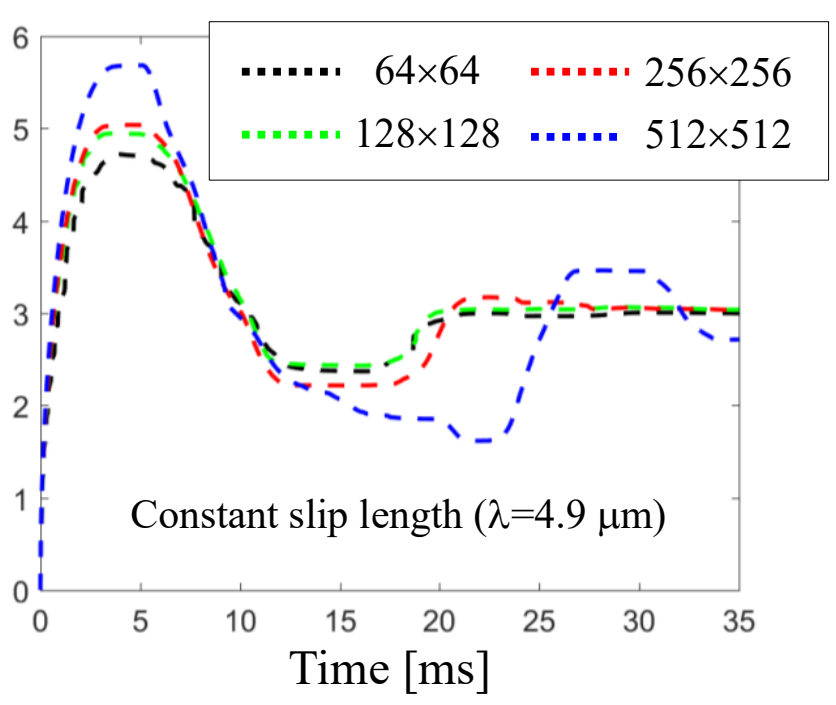

(d)

Fig. 17 


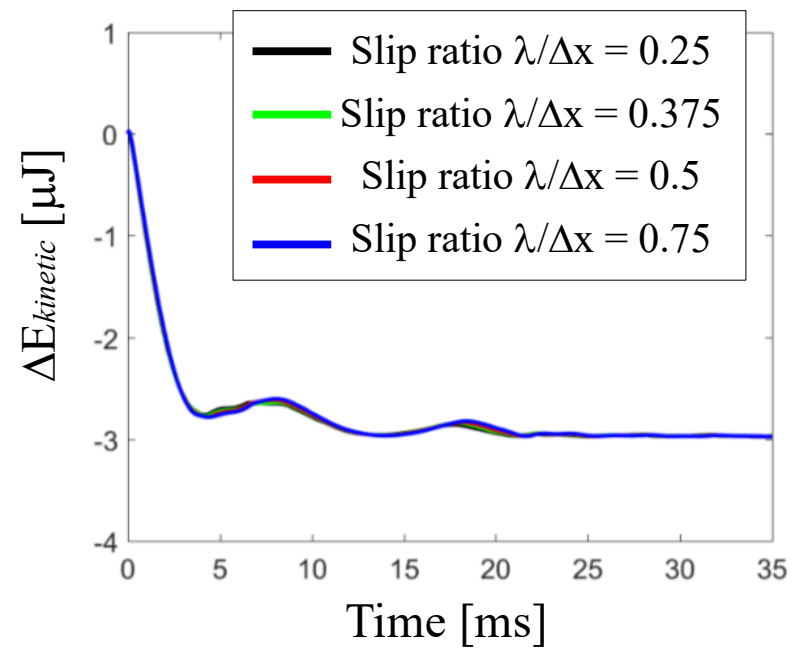

(a)

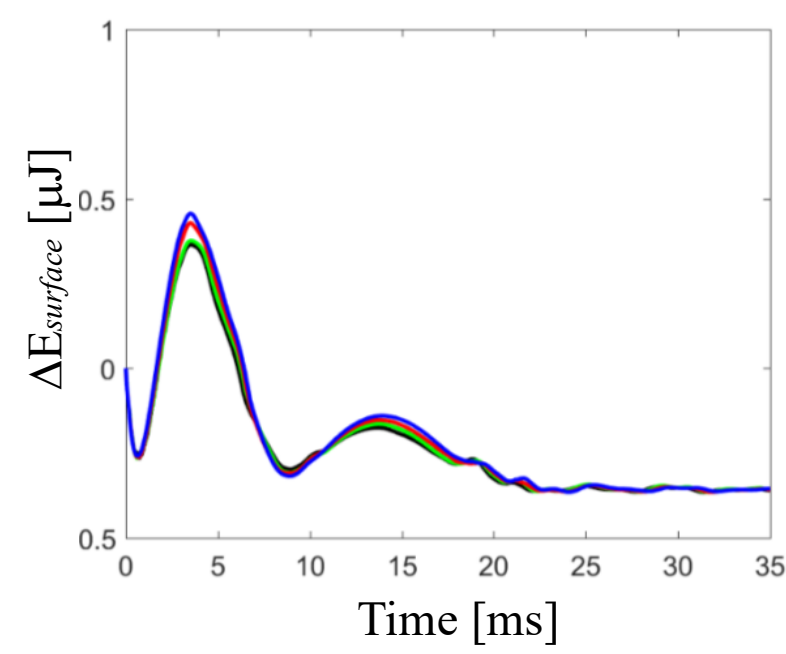

(c)

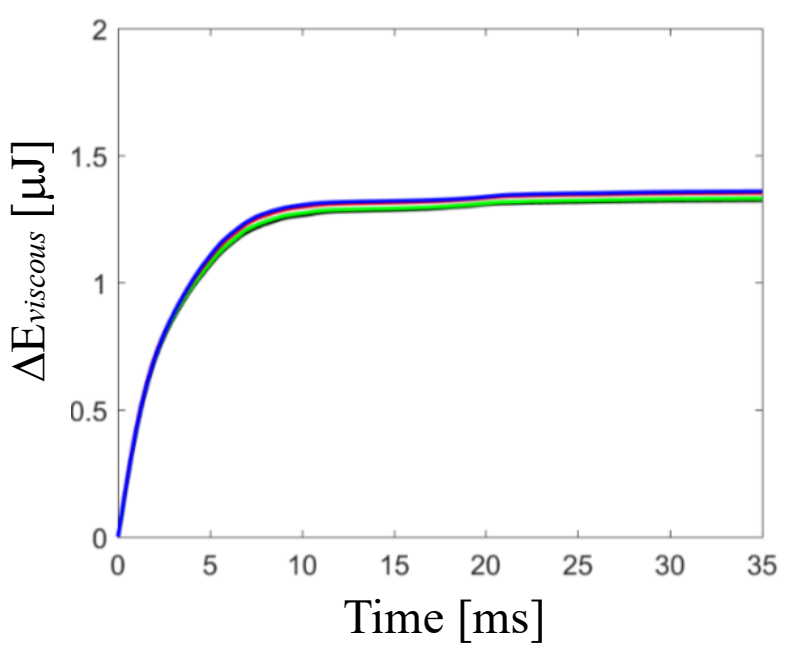

(b)

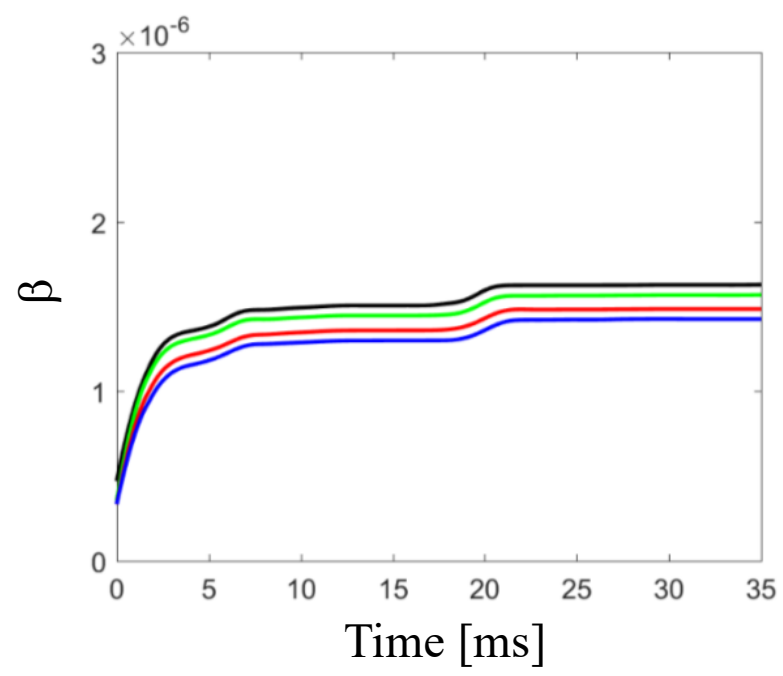

(d)

Fig. 18 


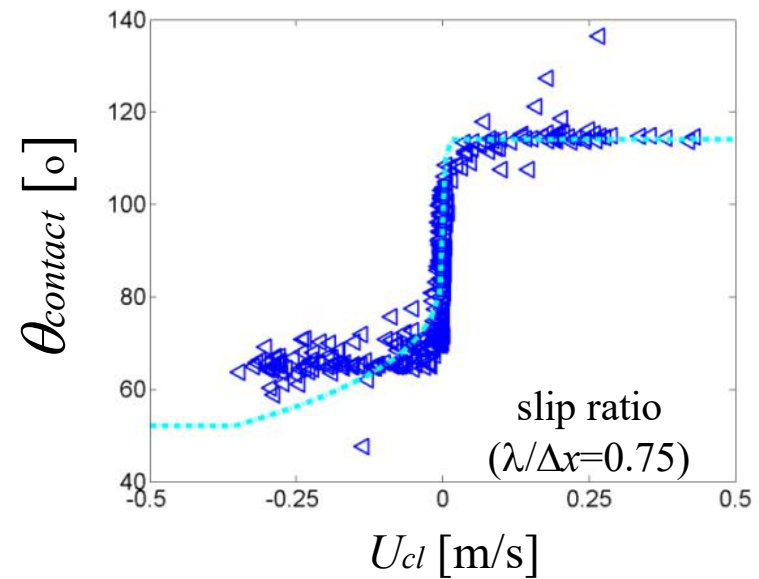

(a)

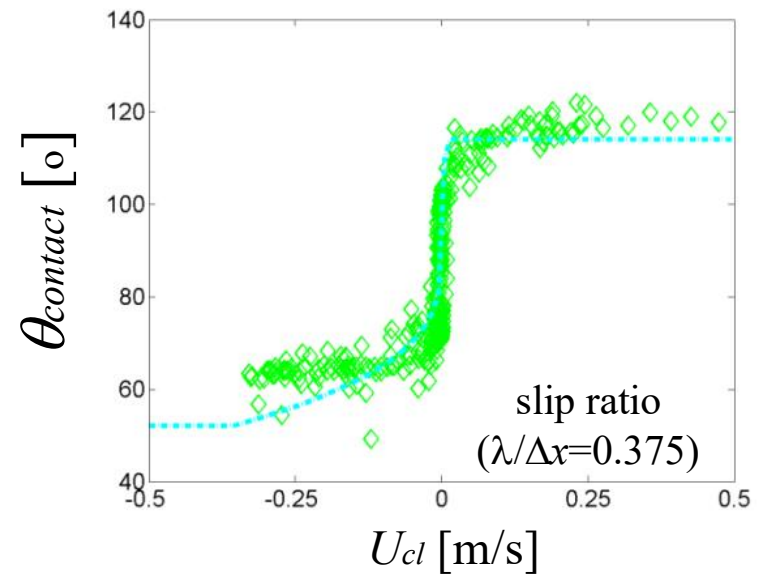

(c)

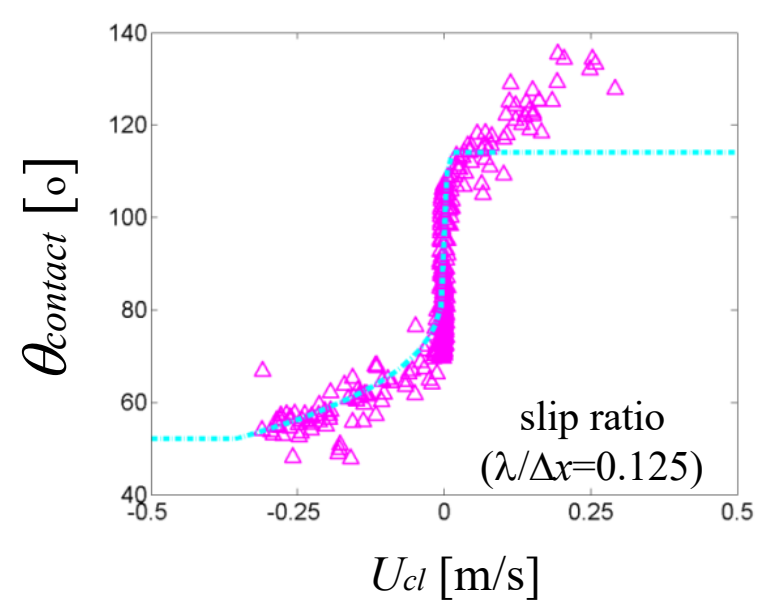

(e)

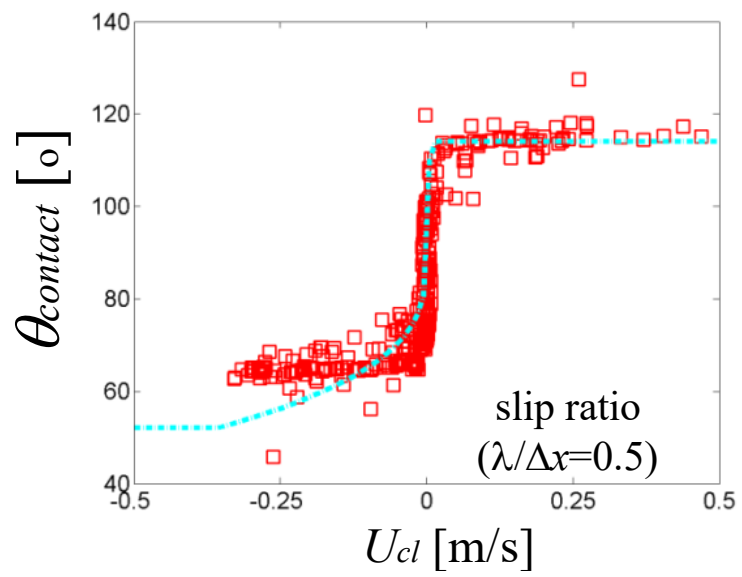

(b)

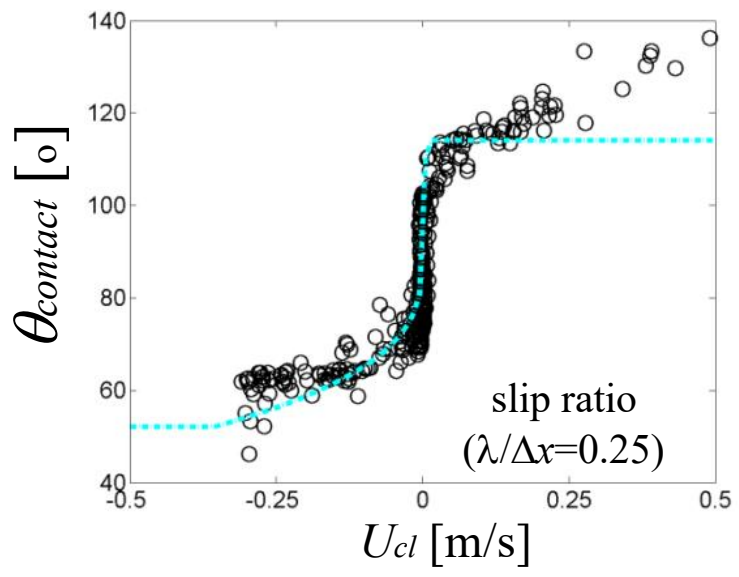

(d)

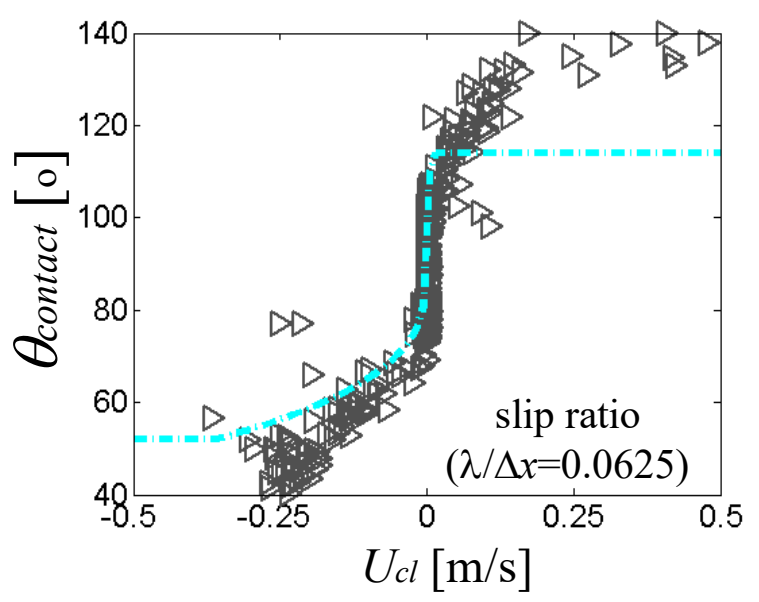

(f)

Fig. 19 


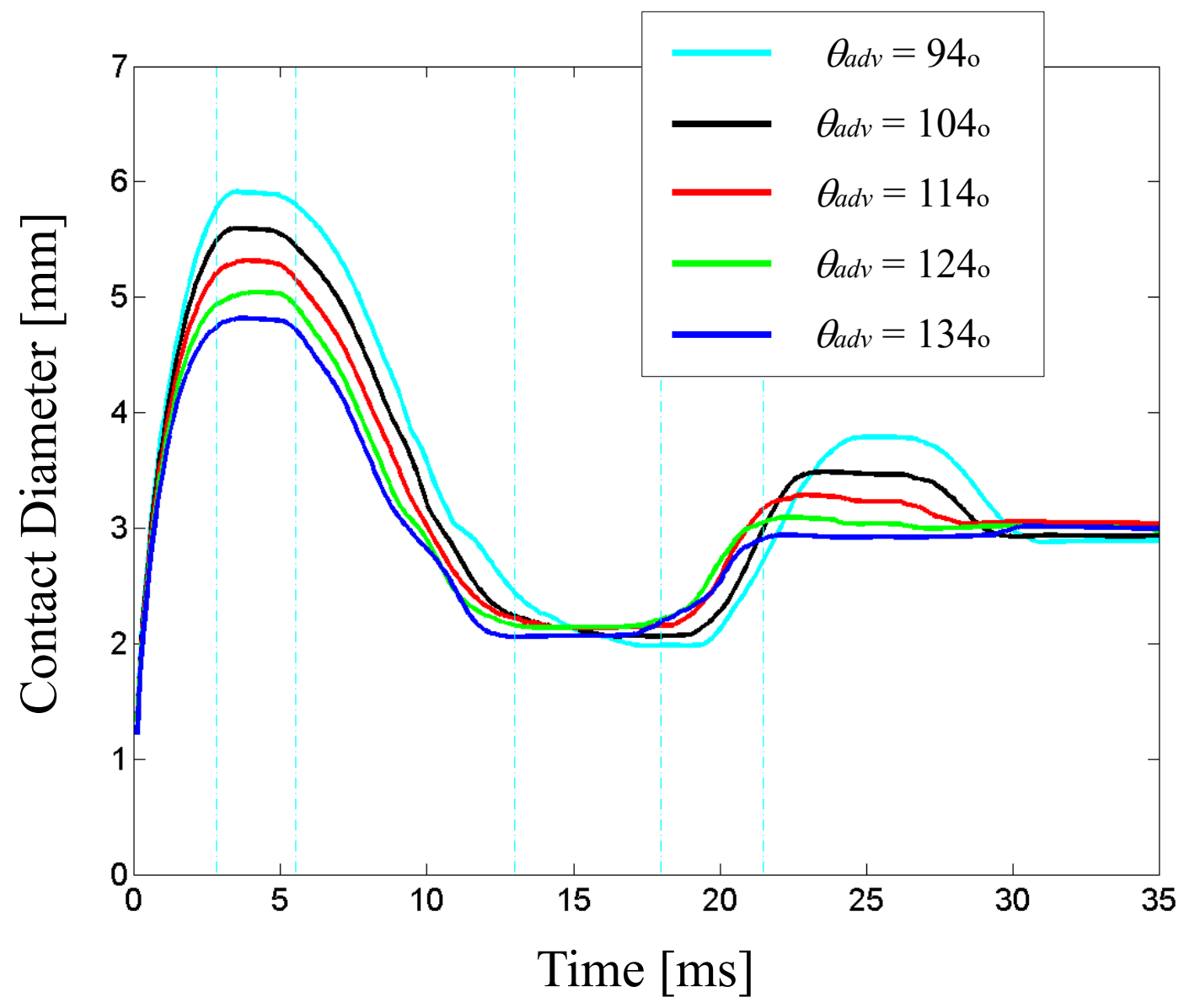

Fig. 20 


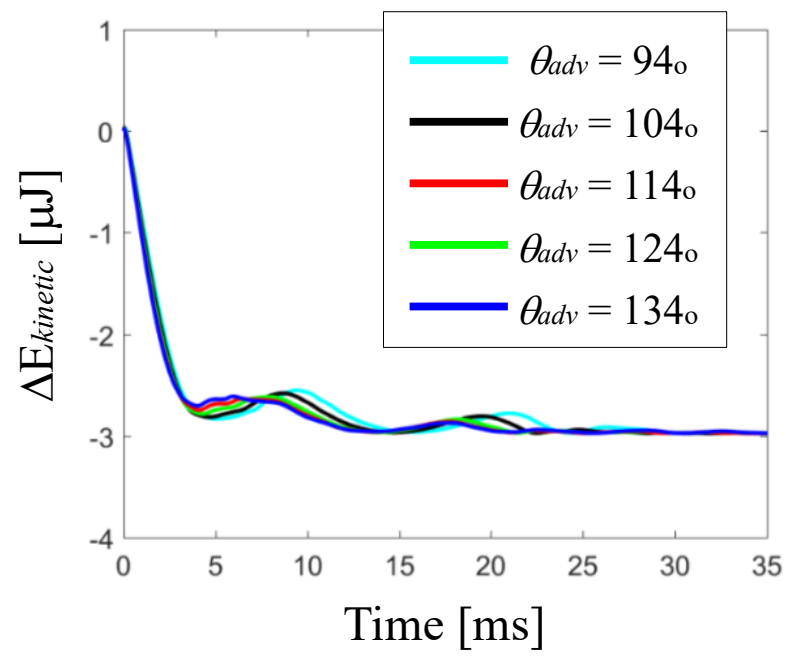

(a)

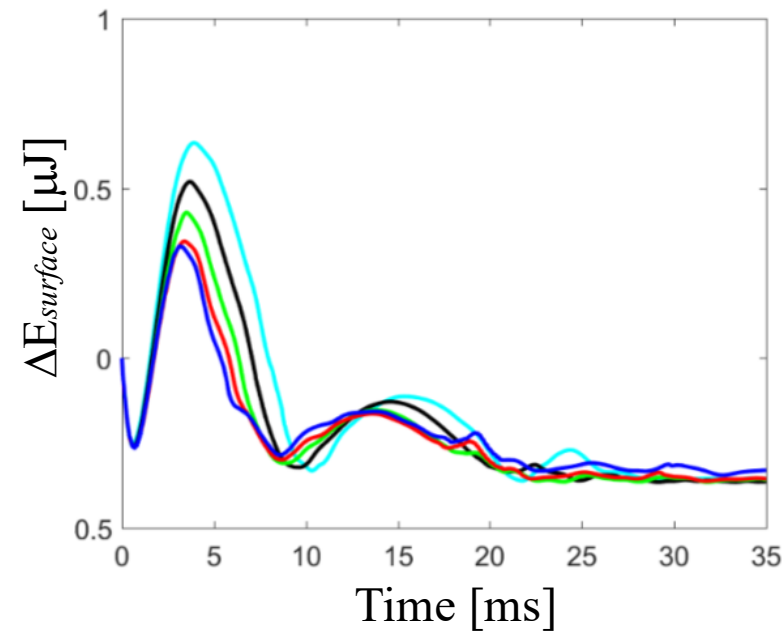

(c)

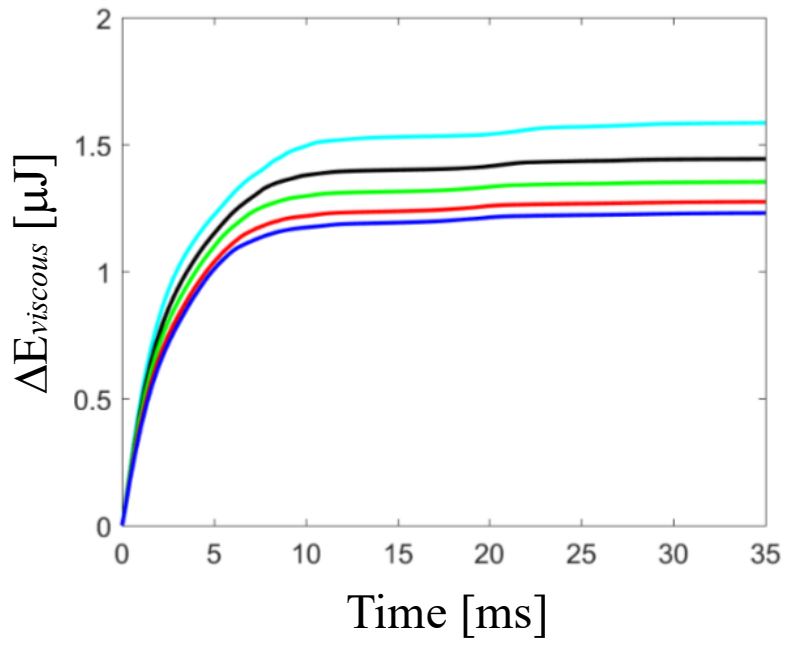

(b)

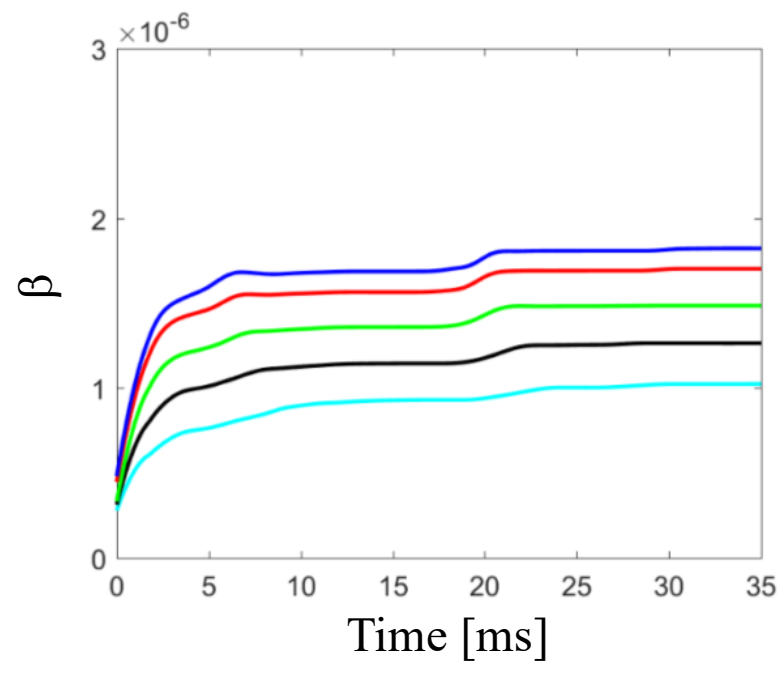

(d)

Fig. 21 


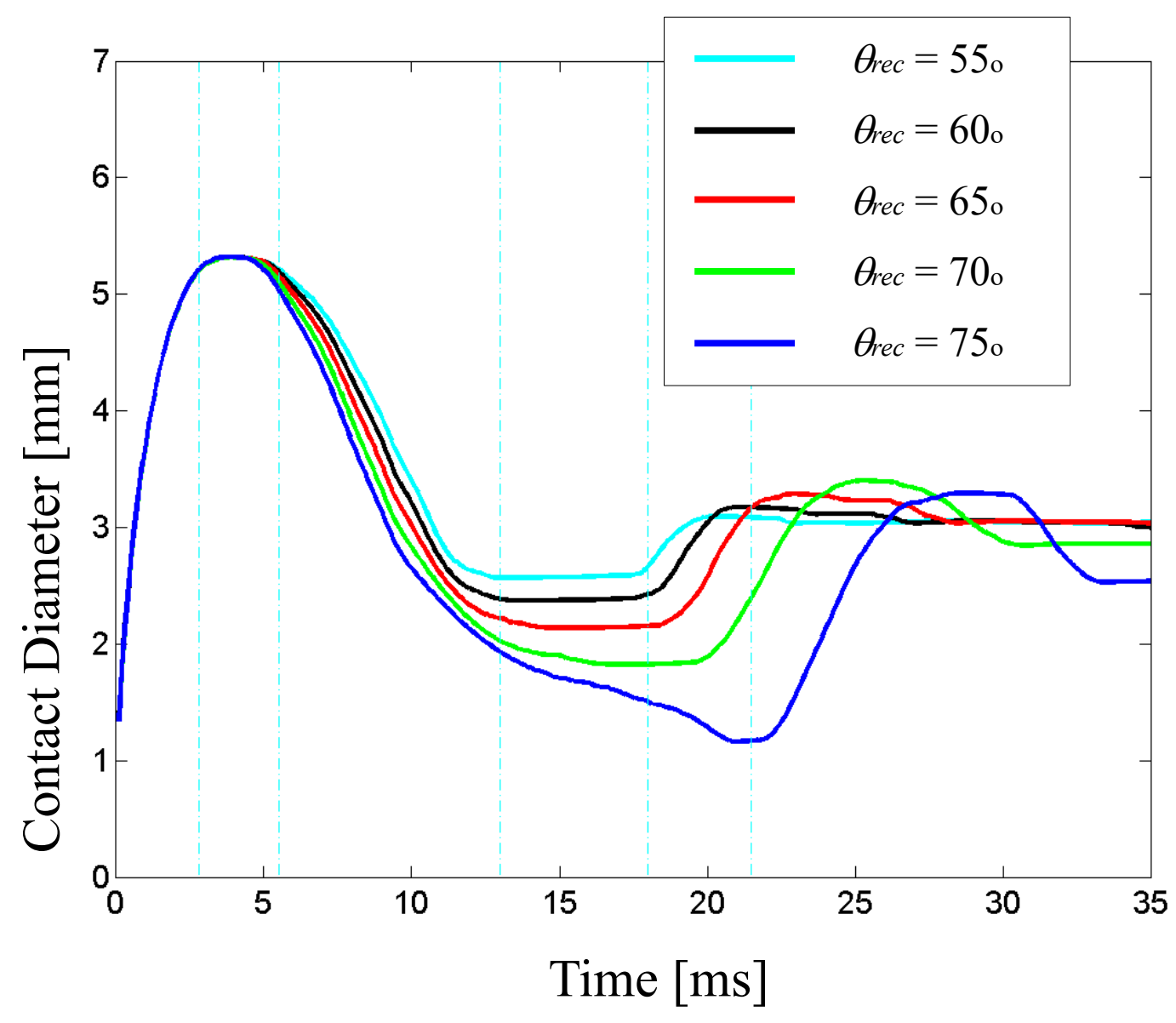

Fig. 22 


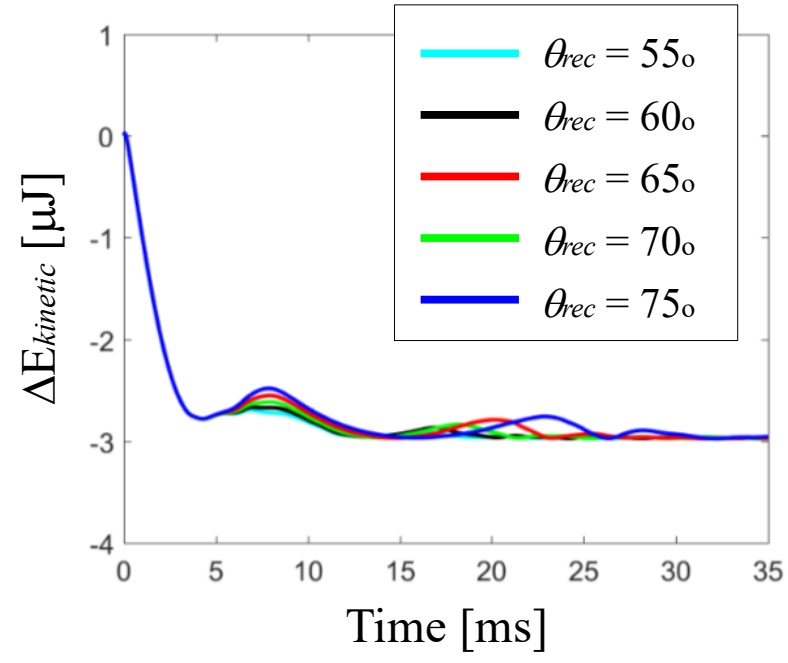

(a)

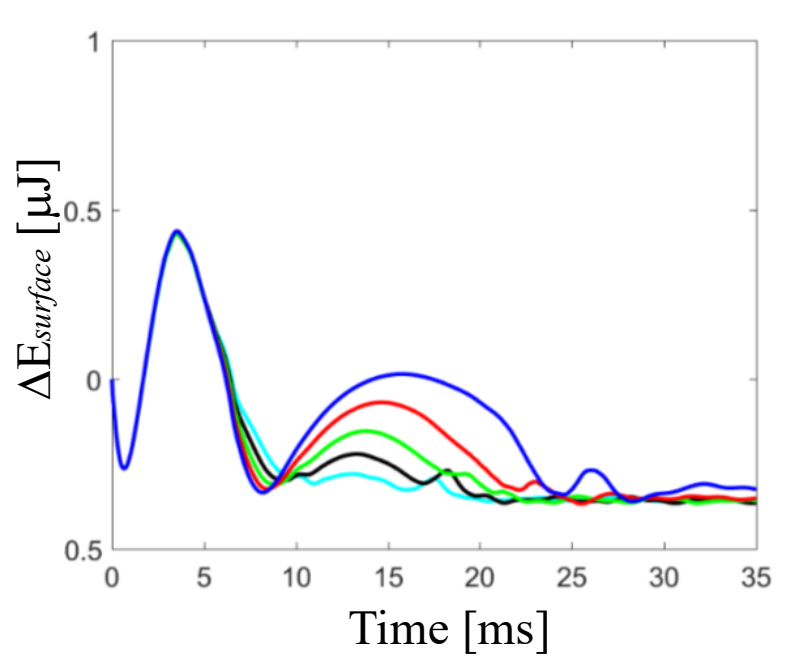

(c)

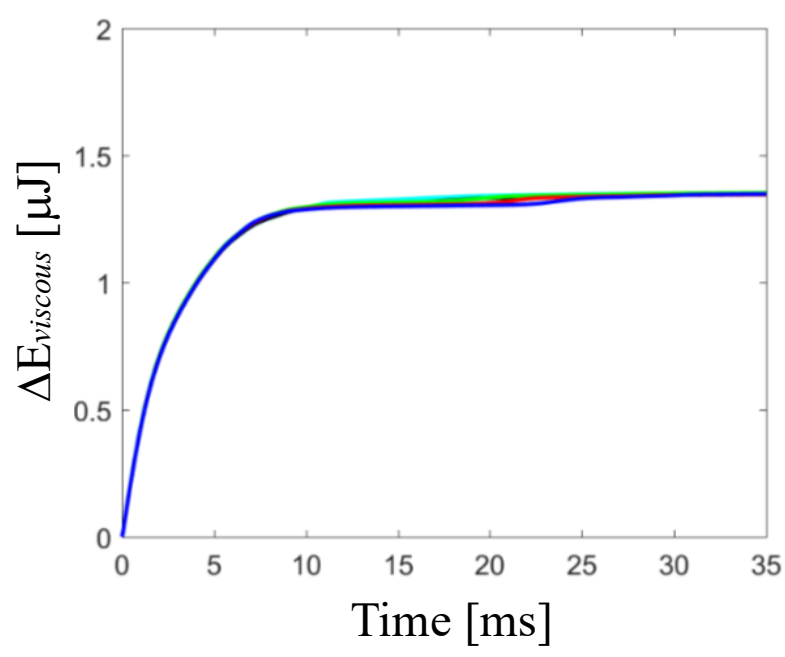

(b)

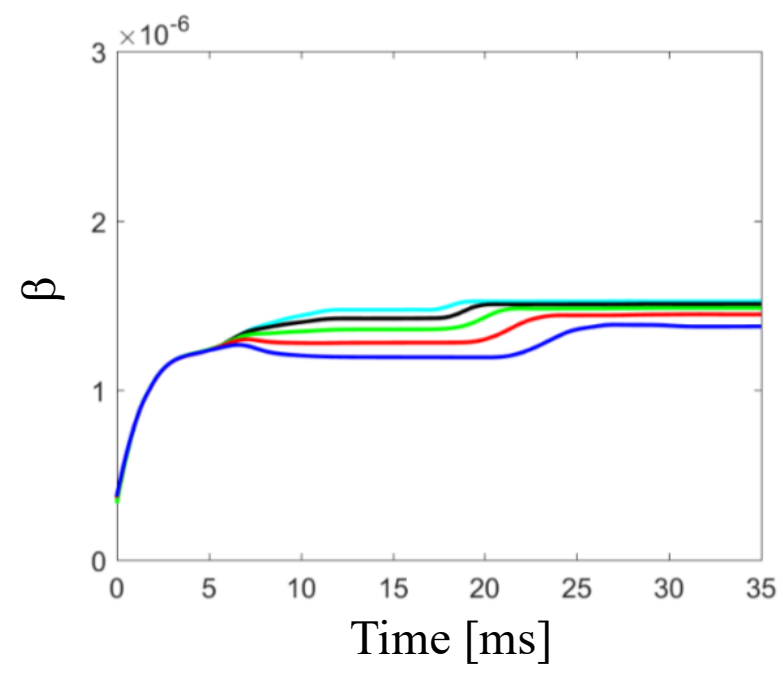

(d)

Fig. 23 


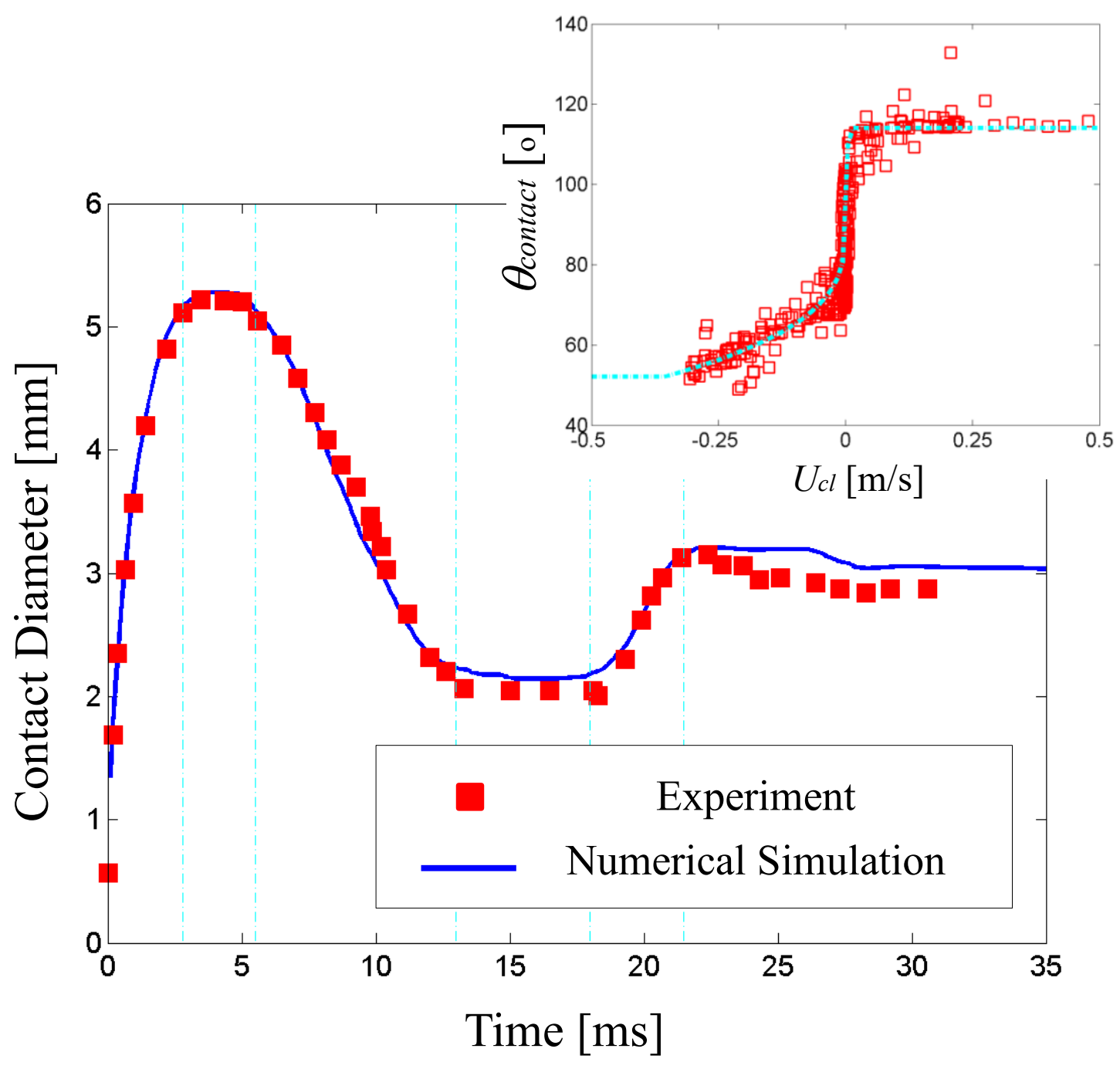

Fig. 24 


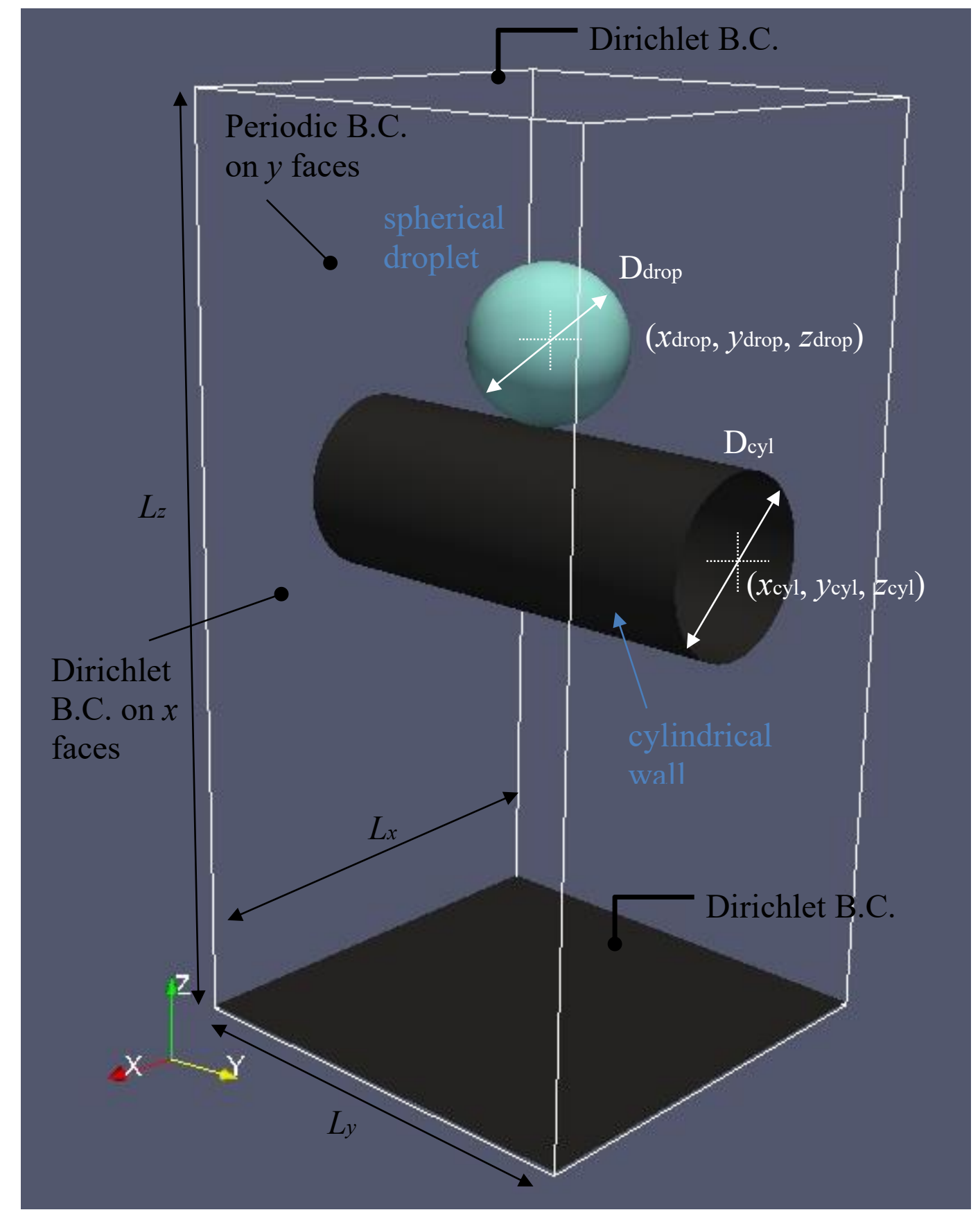

Fig. 25 


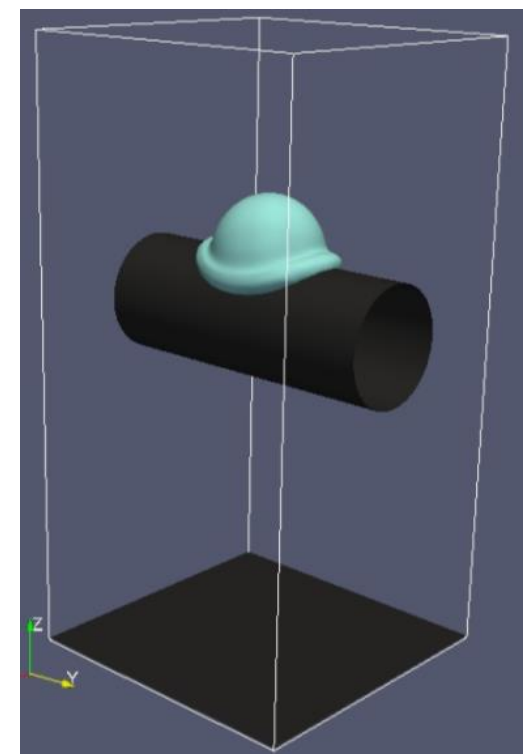

(a) $\mathrm{t}=1.6[\mathrm{~ms}]$

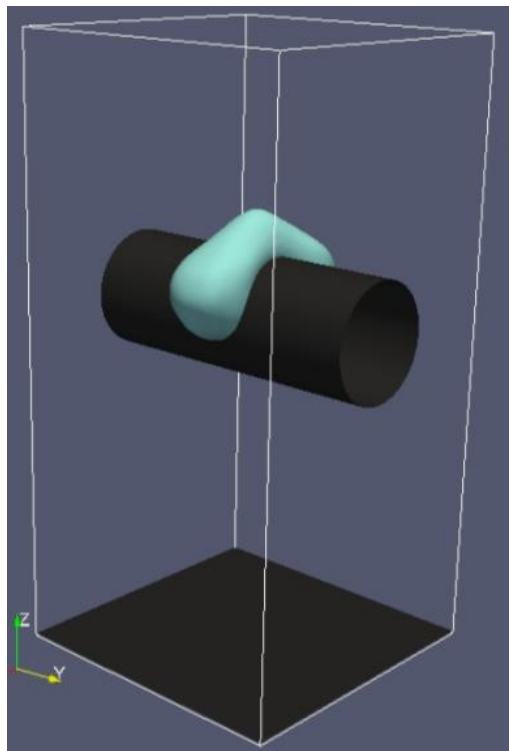

(d) $\mathrm{t}=13.0[\mathrm{~ms}]$

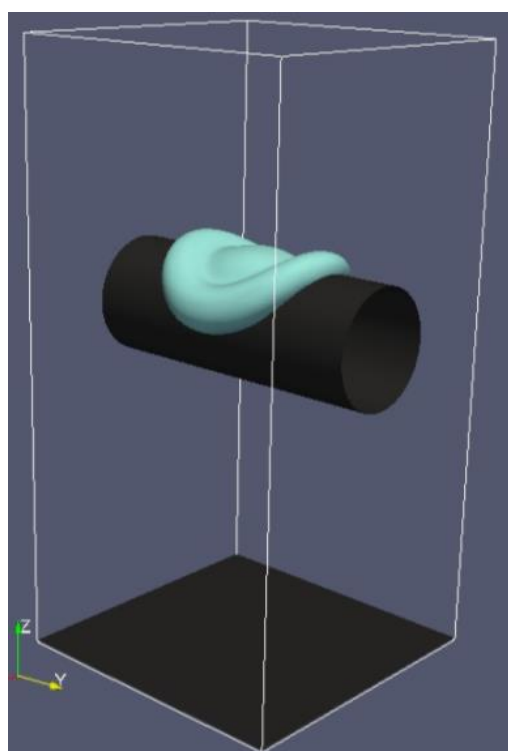

(b) $\mathrm{t}=4.32[\mathrm{~ms}]$

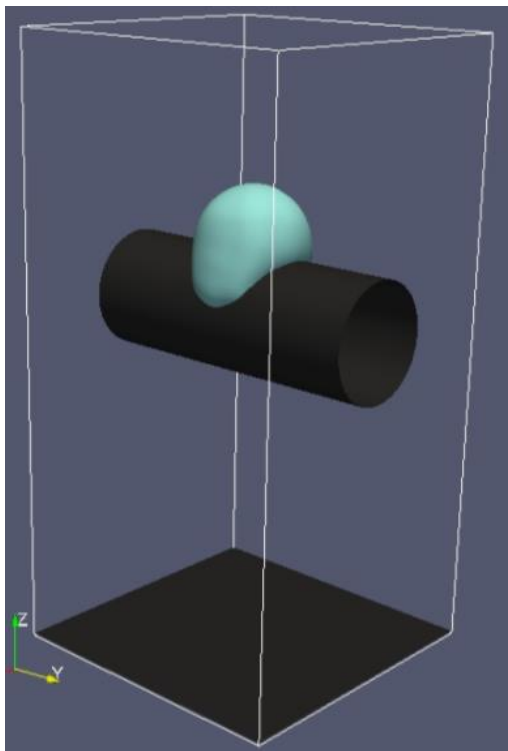

(e) $\mathrm{t}=23.6[\mathrm{~ms}]$

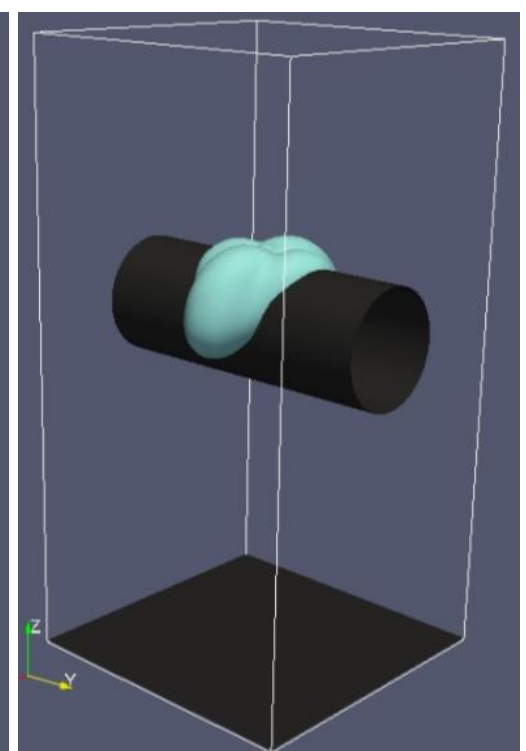

(c) $\mathrm{t}=8.75[\mathrm{~ms}]$

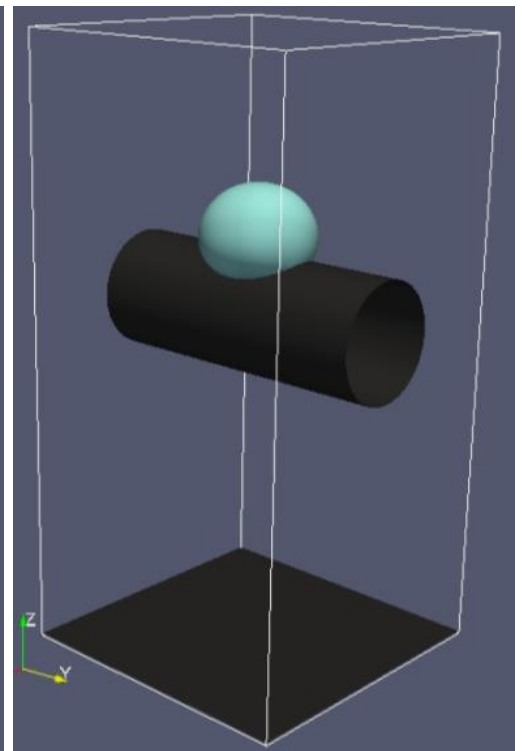

(f) $\mathrm{t}=70.7[\mathrm{~ms}]$

Fig. 26 


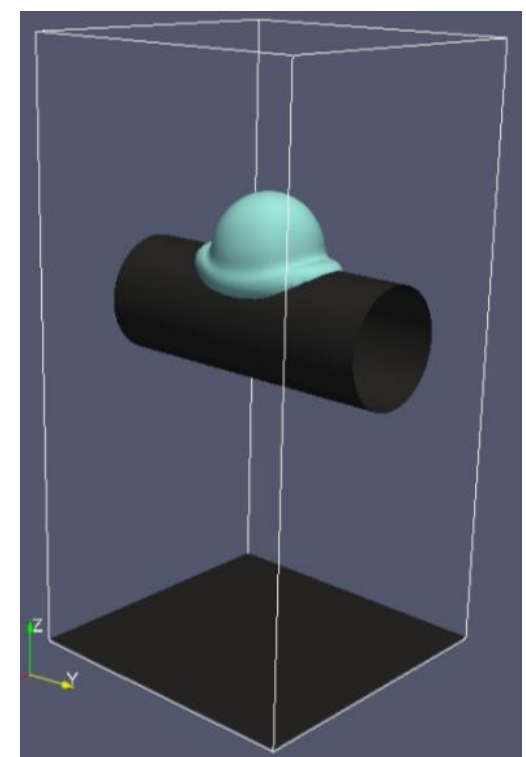

(a) $\mathrm{t}=1.48[\mathrm{~ms}]$

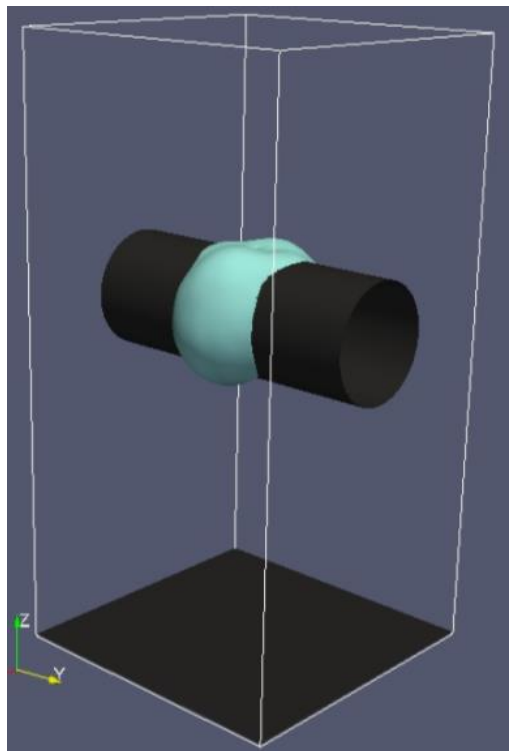

(d) $\mathrm{t}=17.5[\mathrm{~ms}]$

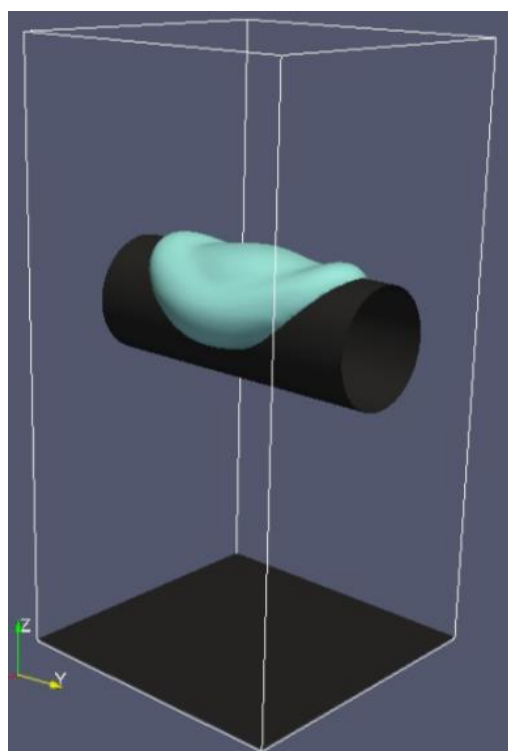

(b) $\mathrm{t}=4.21[\mathrm{~ms}]$

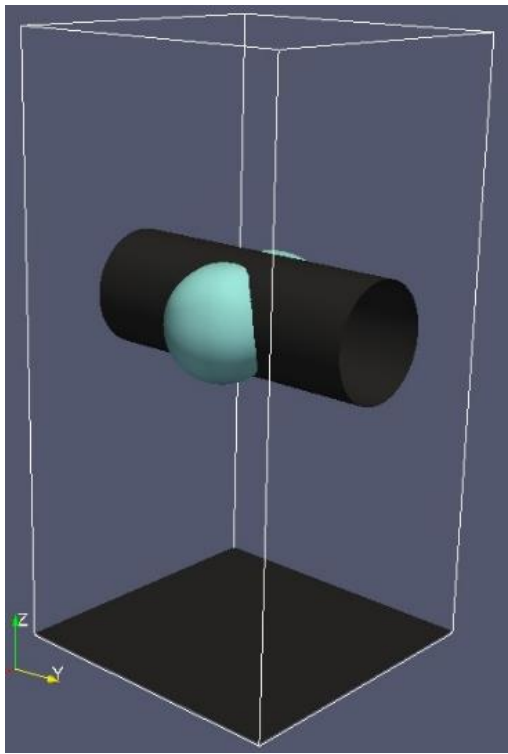

(e) $\mathrm{t}=55.7[\mathrm{~ms}]$

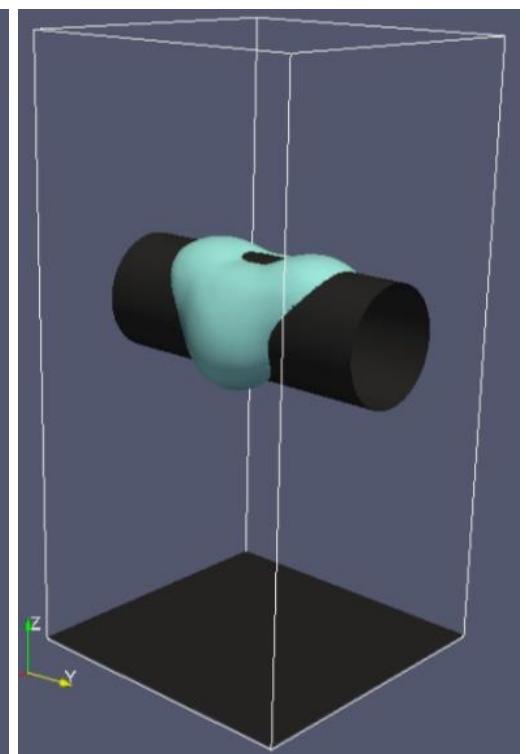

(c) $\mathrm{t}=13.0[\mathrm{~ms}]$

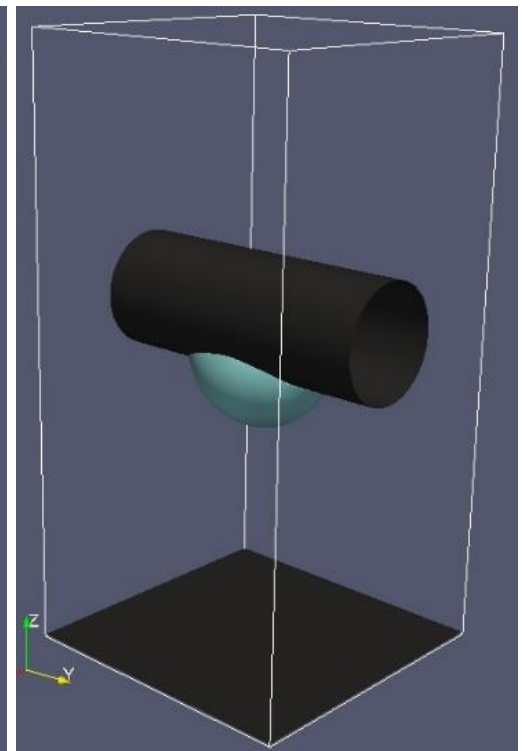

(c) $\mathrm{t}=137[\mathrm{~ms}]$

Fig. 27 


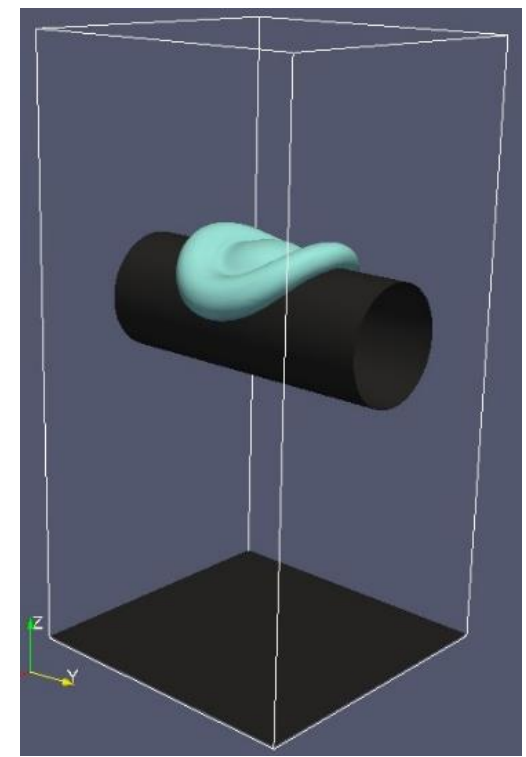

(a) $\mathrm{t}=4.05[\mathrm{~ms}]$

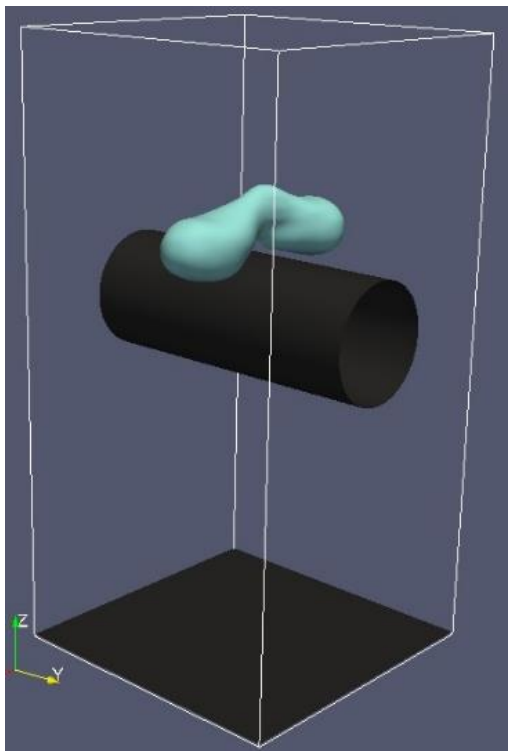

(d) $\mathrm{t}=14.4[\mathrm{~ms}]$

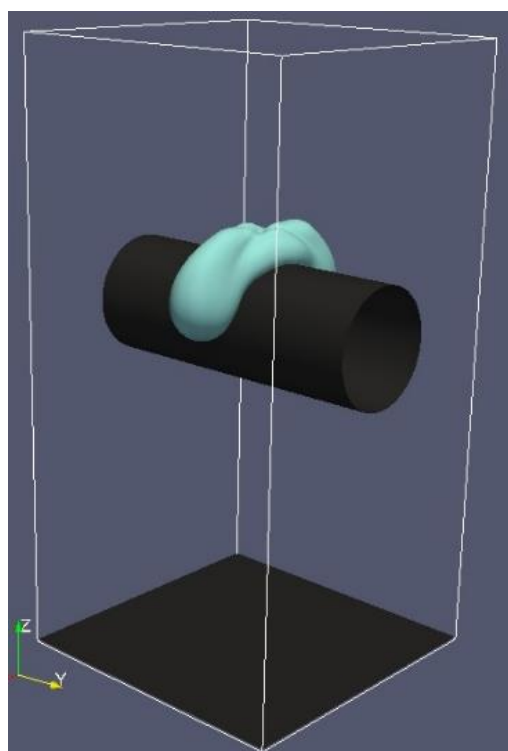

(b) $\mathrm{t}=7.55[\mathrm{~ms}]$

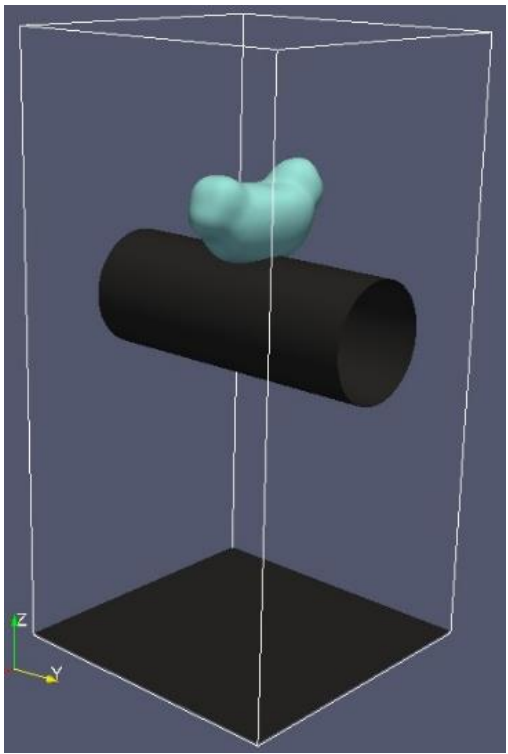

(e) $\mathrm{t}=19.3[\mathrm{~ms}]$

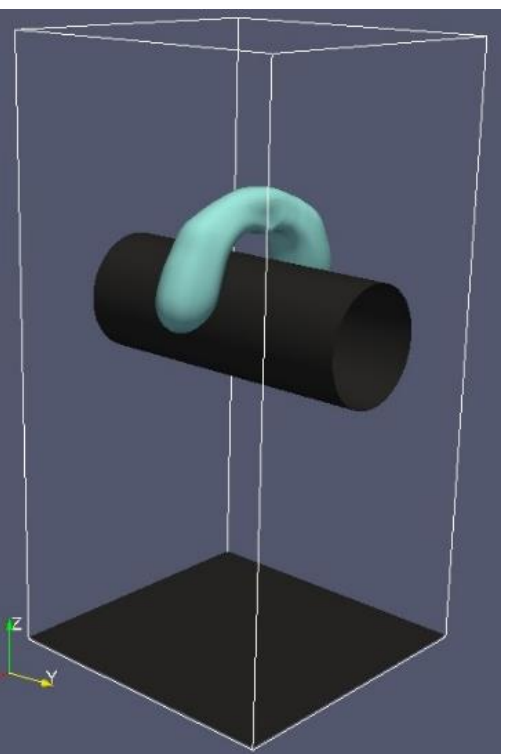

(c) $\mathrm{t}=10.7[\mathrm{~ms}]$

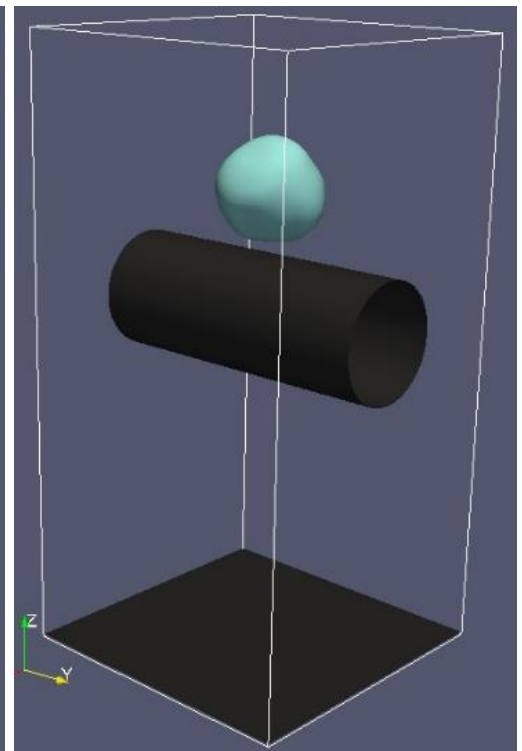

(f) $\mathrm{t}=27.2[\mathrm{~ms}]$

Fig. 28 